\title{
Generación, detección y procesamiento de señales electromagnéticas en la banda de Terahertz
}

Tesis de Doctorado

\section{Federico E. Sanjuan}

Presentada ante la Facultad de Ingeniería de la Universidad Nacional de La Plata como requisito para la obtención del grado académico de

\section{DOCTOR EN INGENIERÍA}

Dirección de tesis:

Director: Jorge O. Tocho.

Codirector: Martin Hurtado.

Jurado de Tesis:

Dr. Pablo A. Costanzo Caso.

Dr. Gustavo A. Torchia.

Dr. Enrique E. Sicre.

Fecha de la defensa oral y pública:

3 de diciembre de 2014. 
A Mariela, mi esposa 


\section{Agradecimientos}

Agradezco a mi familia, amigos, directores, y a todas las personas del Centro de Investigación Opticas, de la Facultad de Ingeniería de la UNLP, y del

Centro de Tecnología Nanofotónica de Valencia, que me han ayudado y acompañado a lo largo de estos años. 


\section{Índice general}

$\begin{array}{ll}\text { Lista de figuras } & \text { V }\end{array}$

$\begin{array}{lll}\text { Lista de tablas } & \text { IX }\end{array}$

$\begin{array}{ll}\text { Acrónimos } & \text { XI }\end{array}$

$\begin{array}{ll}\text { Resumen } & \text { XIII }\end{array}$

1. Introducción 1

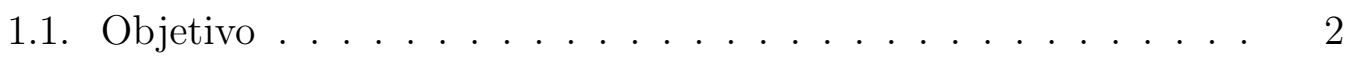

1.2. Esquema General . . . . . . . . . . . . . . . . . 2

2. Conceptos teóricos de generadores y detectores en la banda de terahertz $\quad 5$

2.1. Generadores . . . . . . . . . . . . . . 6

2.1.1. Principales fuentes continuas de $\mathrm{THz} \ldots \ldots$. . . . . . 6

2.1.1.1. Generación por diferencia de frecuencias . . . . 6

2.1.1.2. Mezclado de fotones . . . . . . . . . . 6

2.1.1.3. Multiplicación de frecuencias de microondas . . 6

2.1.1.4. Haz de electrones ondulados periódicamente . . 7

2.1.1.5. Quantum cascade laser (QCL) . . . . . . . 7

2.1.1.6. Láser infrarrojo lejano (FIR) . . . . . . . . . 7

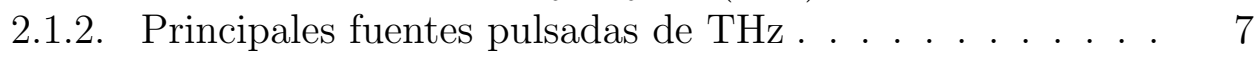

2.1.2.1. Rectificación óptica . . . . . . . . . . . 7

2.1.2.2. Electrones relativistas acelerados (bunch of relativistic electrons ) . . . . . . . . . . 8

2.1.2.3. Plasma en aire inducido por láser . . . . . . . 8

2.1.2.4. Conmutación transitoria fotoconductiva . . . . 9

2.2. Detectores . . . . . . . . . . . . . . . . . . 12

2.2.1. Detección no coherente . . . . . . . . . . . . . . . . . . 12

2.2.1.1. Bolómetros . . . . . . . . . . . . . 12 
2.2.1.2. Piroeléctricos . . . . . . . . . . . . 12

2.2.1.3. Celda de Golay . . . . . . . . . . . . . . . . . . 13

2.2.2. Detección coherente . . . . . . . . . . . . . 13

2.2.2.1. Mezclado de fotones . . . . . . . . . . . . . . 13

2.2.2.2. Detección heterodina . . . . . . . . . . . 13

2.2.2.3. Detección por plasma . . . . . . . . . . 13

2.2.2.4. Muestreo electroóptico y conmutación fotoconductiva .................. 14

3. Desarrollo experimental de un espectrómetro de terahertz re$\begin{array}{ll}\text { suelto en el tiempo } & 21\end{array}$

3.1. Interferómetro de Michelson . . . . . . . . . . . . . . . . . 23

3.1.1. Caracterización del láser de pulsos ultracortos de luz . . 23

3.1.1.1. Introducción . . . . . . . . . . . . . 24

3.1.1.2. Teoría . . . . . . . . . . . 25

3.1.1.3. Simulación Numérica . . . . . . . . . . . . . . 28

3.1.1.4. Experimento . . . . . . . . . . . . . 29

3.1.1.5. Resultados . . . . . . . . . . . . . 30

3.1.1.6. Conclusiones . . . . . . . . . . . . . 34

3.1.2. Montaje del interferómetro . . . . . . . . . . . . . . 34

3.2. Adquisición de datos y alimentación del generador de $\mathrm{THz}$. . . 43

3.2.1. Amplificador de lock-in . . . . . . . . . . . . . . 43

3.2.2. Tensión de alimentación de la antena y adaptador de tensión 46

3.2.3. Software de adquisición . . . . . . . . . . . . . . 46

3.3. Resultados espectrales del vapor de agua . . . . . . . . . . . . . 49

4. Teoría del procesamiento de datos. Resultados espectrales y temporales de materiales $\quad 53$

4.1. Análisis frecuencial . . . . . . . . . . . . . . . . . 53

4.1.1. Resultados . . . . . . . . . . . . . . . . . 56

4.2. Análisis temporal . . . . . . . . . . . . . . . . . . . 56

4.2.1. Resultados . . . . . . . . . . . . . . . . 59

4.3. Errores . . . . . . . . . . . . . . . . 60

5. Método interferométrico de medición de materiales. Resulta$\begin{array}{ll}\text { dos } & 63\end{array}$

5.1. Materiales monocapa ................. . . . 63

5.1.1. Resumen . . . . . . . . . . . . . . 63

5.1.2. Introducción . . . . . . . . . . . . . . . 64

5.1.3. Método . . . . . . . . . . . . . . . 64

5.1.4. Teoría ..................... 65 
5.1.5. Experimento y resultados . . . . . . . . . . . 66

5.1.6. Conclusión . . . . . . . . . . . . . . 68

5.2. Materiales birrefringentes . . . . . . . . . . . . . 68

5.2.1. Resumen . . . . . . . . . . . . . . 68

5.2.2. Introducción . . . . . . . . . . . . . . . . . . 68

5.2.3. Teoría y simulación . . . . . . . . . . . . . . . 70

5.2.4. Experimento y resultados . . . . . . . . . . 74

5.2.5. Conclusión . . . . . . . . . . . . 77

5.3. Materiales multicapa . . . . . . . . . . . . . . . 77

5.3.1. Resumen . . . . . . . . . . . . . 77

5.3.2. Introducción . . . . . . . . . . . . . . . . . . . . 77

5.3.3. Teoría y simulación . . . . . . . . . . . . . . . 78

5.3.4. Experimento y resultados . . . . . . . . . . . . . . 81

5.3.5. Conclusión . . . . . . . . . . . . . . 83

6. Conclusiones y posibilidades futuras $\quad 85$

6.1. Conclusiones . . . . . . . . . . . . . . . 85

6.2. Posibilidades futuras . . . . . . . . . . . . . . . 86

$\begin{array}{ll}\text { A. Anexo I: Programa de adquisición de datos } & 87\end{array}$

$\begin{array}{ll}\text { Bibliografía } & 91\end{array}$ 


\section{Índice de figuras}

1.1. Espectro electromagnético. . . . . . . . . . . . . . . . . . 1

2.1. Generación por diferencia de frecuencias. . . . . . . . . . 6

2.2. Mezclado de fotones. . . . . . . . . . . . . . . . . . . 6

2.3. Multiplicación de frecuencias de microondas. . . . . . . . . . . . 7

2.4. Rectificación óptica. . . . . . . . . . . . . . . . . . . 8

2.6. Esquema de bandas de un semiconductor cerca de la superficie. $\quad 9$

2.5. Plasma en aire inducido por láser. . . . . . . . . . . . . . . . . 9

2.7. Esquema de la emisión de $\mathrm{THz}$ de una antena fotoconductora polarizada excitada por un láser de fs. . . . . . . . . . . . 10

2.8. Configuraciones de electrodos de antenas fotoconductoras. . . . 11

2.9. Antena generadora stripline (Marca Teravil). . . . . . . . . . . . 12

2.10. Configuración experimental para detección por plasma. . . . . . 14

2.11. Configuración típica de un espectrómetro de THz. . . . . . . . . 15

2.12. Configuración típica de detección por muestreo electro-óptico. . 15

2.13. Principio de detección por muestro electro-óptico con y sin presencia de THz. . . . . . . . . . . . . . . . . . 16

2.14. Mapeo de la detección del campo eléctrico. . . . . . . . . . . . 17

2.15. Dimensiones de antena fotoconductora. . . . . . . . . . . . . . 17

2.16. Antena detectora dipole offset (marca Teravil). . . . . . . . . . . 19

3.1. Configuración típica de un espectrómetro de THz. . . . . . . . . 22

3.2. Esquema de emisión de radiación debido a TPA. . . . . . . . . . 25

3.3. Configuración contrapropagante de los pulsos láser. . . . . . . . 26

3.4. (a) Resultado de simular la ecuación (3.15). (b) Resultado de la convolución de la Fig. 3.4(a) con una función Gaussiana de $F W H M=1,145 \mu \mathrm{m}$ (resolución de la cámara). . . . . . . . . . 
3.5. Configuración del espectrómetro de Michelson montado; E.1, E.2, E.3, E.4, E.5, E.6, E.7 son los espejos; B.S. es el beam splitter; L.1, L. 2 son las lentes de $5 \mathrm{~cm}$ de distancia focal; L.R. es la línea de retardo, Mo. es el motor paso a paso; C. es la celda de Rhodamina B donde los pulsos se encuentran. (a) Esquema gráfico. (b) Montaje experimental. . . . . . . . . . . . . . 30

3.6. Características del láser Mai Tai (Spectra Physics). . . . . . . . 31

3.7. Características del motor paso a paso DMX UMD. . . . . . . . . 32

3.8. Fotografía con presencia de scattering. . . . . . . . . . . . 32

3.9. (a,b) Imágenes de los pulsos solapadados en distintas posiciones. 33

3.10. Promedio espacial de la suma de las dos imágenes de la Fig. 3.9. 33

3.11. Aproximación de la emisión de fluorescencia de la Fig. 3.9(a) por una función Gaussiana. . . . . . . . . . . . . . . . . . . . . 34

3.12. Medición del ancho del pulso temporal a través del uso del equipo comercial FROG. . . . . . . . . . . . . . . . .

3.13. Configuración experimental del espectrómetro. (a) Esquema gráfico. (b) Foto del montaje experimental. . . . . . . . . . . . 36

3.14. Gráfico de la ecuación (3.18) para distintos valores de frecuencia. 38

3.15. Lente colimadora de silicio con la realización de la traza de rayos. 38

3.16. Lente de silicio Hyper-hemispherical de antena Teravils. . . . . . 39

3.17. Configuraciones de lentes. . . . . . . . . . . . . . . . 40

3.18. Configuración con espejos parabólicos. . . . . . . . . . . . . 40

3.19. (a) Imagen del área de enfoque de la antena generadora. (b) Imagen del área de enfoque de la antena detectora. . . . . . . . . . 41

3.20. (a) Fotografía del enfoque logrado en la antena generadora. (b) Fotografía del enfoque logrado en la antena detectora. . . . . . . 41

3.21. Características del amplificador de lock-in extraídas del manual del fabricante. . . . . . . . . . . . . . . . . 44

3.22. Señal del experimento modulada y señal de referencia del lock-in. 45

3.23. (a) Esquemático de la fuente de tensión. (b) PCB. (c) Foto del circuito realizado. . . . . . . . . . . . . . 47

3.24. (a) Esquemático del adaptador de tensión. (b) PCB. (c) Foto del circuito realizado. . . . . . . . . . . . . . . .

3.25. (a) Traza temporal del vapor de agua. (b) Espectro del vapor de agua. . . . . . . . . . . . . . . . 51

4.1. Esquema de la geometría de espectroscopía por transmisión. . . 54

4.2. Esquema de la geometría de espectroscopía por transmisión. . . 55

4.3. (a) Señal temporal de una muestra de zafiro. (b) Espectro de la traza temporal de (a). . . . . . . . . . . . . . 57 
4.4. (a) Índice de refracción de una muestra de zafiro. (b) Índice de refracción de una muestra de sílica. . . . . . . . . . . . . . . 58

4.5. Resultados temporales. . . . . . . . . . . . . . . . . . . 59

4.6. Varianza de los parámetros ópticos. . . . . . . . . . . . . . 61

5.1. Diagrama en bloques del procesamiento de datos. . . . . . . . 65

5.2. Medidas en muestras de sílica. (a) Señal temporal del espectrómetro de la muestra de sílica de $1.21 \mathrm{~mm}$. (b) Espectro de la señal luego de realizar el valor absoluto de la DFT de la Fig. 5.2(a). (c) La línea azul corresponde al análisis espectral de la Fig. 5.2(b); Las líneas negra y roja son el resultado final del procesamiento en las muestras de $1.97 \mathrm{~mm}$, y $3.1 \mathrm{~mm}$ respectivamente. . . . .

5.3. Proyección del campo eléctrico de $\mathrm{THz}$ sobre el material birrefringente. . . . . . . . . . . . . . . . .

5.4. Simulación numérica de la DFT aplicada a la señal frecuencial de la ecuación (5.6). . . . . . . . . . . . . . . . . .

5.5. Simulación numérica de la DFT aplicada a la señal de la ecuación (5.6) para distintos ángulos de la muestra. . . . . . . . . . . 74

5.6. Configuración experimental del espectrómetro de THZ. . . . . . 75

5.7. (a) Medición temporal del espectrómetro de THz con una ventana de zafiro de $1 \mathrm{~mm}$ de espesor. (b) Es el módulo de la DFT aplicada a la señal en (a). (c) Es el módulo al cuadrado de la DFT de la señal en (b). . . . . . . . . . . . . . . .

5.8. Medidas de la DFT del módulo del espectro rotando la muestra cada 5 grados. . . . . . . . . . . . . . . . . . . 76

5.9. Traza de rayos del campo eléctrico en una estructura bicapa. . . 78

5.10. Simulación numérica de la transformada discreta de Fourier aplicada a la ecuación (5.15). . . . . . . . . . . . . . . . .

5.11. (a) Traza temporal medida del espectómetro de THz. (b) Resultado de aplicar el procesamiento propuesto a la traza temporal mostrada en (a). . . . . . . . . . . . . . . . . 


\section{Índice de tablas}

3.1. Líneas espectrales del agua . . . . . . . . . . . . . 5 50

4.1. Resultados. . . . . . . . . . . . . . . . . . . 59 


\section{Acrónimos}

FTIR Fourier transform infrared spectroscopy

TDS espectroscopía resuelta en el tiempo

PUL pulsos ultracortos de luz

PCA antena fotoconductora

BWO backward wave oscillators

FELs láseres de electrones libres

QCL láser de cascada cuántica

FIR láser infrarrojo lejano

TPA absorción de dos fotones

FWHM ancho a mitad de altura

FROG frequency resolved optical gating

SHG generación de segunda armónica

DFT transformada de Fourier discreta

NIR infrarrojo cercano

XRD difracción de rayos $\mathrm{x}$ 


\section{Resumen}

La banda de los terahertz $(\mathrm{THz})$ ha sido una de las regiones menos estudiadas del espectro electromagnético debido a la falta de fuentes y detectores. No obstante, desde mediados de los ochenta con los avances tecnológicos se comenzaron a desarrollar sistemas que permitían generar y detectar dichas señales. Los mismos, fueron útiles para realizar espectroscopía que es la aplicación que suscita mayor interés. Esto es debido a que trabajar en la región de los $\mathrm{THz}$ posibilita estudiar materiales de manera no invasiva, y no ionizante. También lo es, porque contribuye a aplicaciones en imágenes que es una herramienta que ya se esta utilizando como medio de diagnostico médico, o como sistema de seguridad entre otras cosas.

En el trabajo de tesis se desarrolló un espectrómetro de $\mathrm{THz}$ mediante el uso de un láser de pulsos ultracortos de luz y antenas fotoconductoras. Para su realización, se desarrollaron piezas mecánicas e implementaciones de hardware y software. También, se propuso una técnica de medición del ancho del pulso láser necesaria para asegurar el correcto funcionamiento del sistema.

Finalizado el montaje del espectrómetro, se realizaron medidas de materiales verificando el buen funcionamiento del mismo.

En una segunda parte, se innovaron en técnicas de procesamientos de datos para materiales monocapa, birrefringente, y multicapa. Tales enfoques, valieron tres publicaciones en revistas internacionales. 


\section{Capítulo 1}

\section{Introducción}

La banda de terahertz en el espectro electromagnético comprende la región que se encuentra entre la banda de las microondas y el infrarrojo (Fig. 1.1). Como no se ha determinado un estándar para definir los límites frecuenciales de dicha banda [1, 2], en el presente trabajo se especifican entre 0.1-10 THz, o expresado en longitudes de onda entre $3 \mathrm{~mm}$ y $30 \mu \mathrm{m}$ [1].

Dicha región despierta gran interés científico, debido a que ocurren fenómenos físicos en la misma, tales como las transiciones rotacionales de las moléculas, las vibraciones de las redes en sólidos, las transiciones intrabanda en semiconductores, las energías de gap en superconductores, etc. [1].

También es interesante para el campo astronómico, debido a que la radiación de terahertz llega naturalmente desde el espacio aportando datos sobre la radiación de fondo [3].

Sin embargo, por falta de generadores y detectores artificiales ha sido la banda menos explorada. Tal situación, fue consecuencia de que los desarrollos tecnológicos vinculados a las microondas han sido limitados por las velocidades de operación de los dispositivos electrónicos [4], y por la banda proveniente del infrarrojo, por la falta de fuentes láseres que operen en dicho rango.

El único sistema que operaba en la banda de los terahertz hasta la década del 80, era la técnica interferométrica Fourier transform infrared spectroscopy (FTIR). El cambio fue provocado por David. H. Auston [5], junto a sus

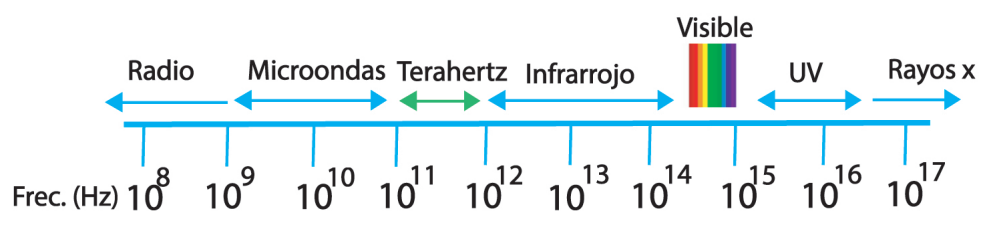

Figura 1.1: Espectro electromagnético. 
compañeros de trabajo, que lograron innovar con una técnica espectroscópica conocida actualmente como espectroscopía resuelta en el tiempo (TDS) en 1980. La misma, fue lograda mediante la conjunción de dispositivos electrónicos y fotónicos. TDS, permitió estudiar la banda de $\mathrm{THz}$ detectando por primera vez el campo eléctrico de manera coherente (intensidad y fase), proporcionando como inmediata y principal aplicación el desarrollo de la espectroscopía.

En comparación con FTIR, TDS resuelve con mejor resolución temporal, tiene mayor relación señal ruido por debajo de los $3 \mathrm{THz}$ y al medir de manera coherente el campo eléctrico, es directo el cálculo de la absorbancia e índice de refracción. En cambio, mediante FTIR se obtiene solo la intensidad, lo que lleva a utilizar las transformaciones de Kramers-Kronig para el cálculo de los parámetros ópticos [6].

En los últimos 30 años, se han podido realizar otras formas de generación y detección de ondas de terahertz, así como diferentes procesamientos de datos que han desarrollado el campo hasta lograr aplicaciones en industria [7], imágenes [8], seguridad [9], etc.

\subsection{Objetivo}

El propósito central de la tesis fue realizar la generación y detección de señales en la banda de terahertz empleando pulsos ultracortos de luz (PUL) y antenas fotoconductoras (PCA). Asimismo lo fue la construcción de un espectrómetro de $\mathrm{THz}$, y el desarrollo de alternativas en el procesamiento de datos, para poder implementar variantes y mejoras en el cálculo de los índices de refracción de diferentes materiales y estructuras.

\subsection{Esquema General}

La tesis se divide fundamentalmente en dos partes. En la primera parte (Cap. 2-3) se desarrollan los elementos que componen el espectrómetro de $\mathrm{THz}$, finalizando con la implementación adoptada y con resultados espectrales obtenidos. La segunda parte (Cap. 4-5), esta dedicada al procesamiento de datos desarrollado para la obtención de los parámetros ópticos de los materiales utilizando técnicas ya conocidas, y otras novedosas que resultaron de nuestro trabajo de investigación.

El capítulo dos realiza una descripción teórica de las distintas clases de generadores y detectores de $\mathrm{THz}$, finalizando con los utilizados en el trabajo experimental.

El capítulo tres se centra en el desarrollo experimental del espectrómetro 
de $\mathrm{THz}$, donde se discute y analiza al mismo en dos partes fundamentales. La primera, describe todo lo referente al montaje del interferómetro de Michelson, donde en particular se propone un método de medición de pulsos ultracortos de luz [10]. La segunda, trata sobre los elementos electrónicos y de software necesarios para la generación, medición, y adquisición de datos de la radiación de THz. Finalmente, el capítulo concluye con los resultados espectrales del vapor de agua medidos con el espectrómetro desarrollado.

El capítulo cuatro presenta el marco teórico por el cual se obtienen los parámetros ópticos de las muestras, y se desarrollan técnicas de procesamiento de datos ya conocidas para la obtención de espectros mostrando resultados conseguidos.

En el capítulo cinco se desarrollan las técnicas novedosas de procesamiento de datos para la obtención de los índices de refracción de muestras monocapa [11], birrefringentes [12] y estructuras multicapa [13].

Por último, en el capítulo seis se presentan las conclusiones y posibles trabajos futuros. 


\section{Capítulo 2}

\section{Conceptos teóricos de generadores y detectores en la banda de terahertz}

\section{Resumen}

Una de las primeras señales de $\mathrm{THz}$ que existieron a lo largo de nuestra historia, fue provocada por las radiaciones recibidas desde el espacio debido a las emisiones de sistemas que se encuentran a temperaturas próximas a los 10 K. Dichas señales, fueron difíciles de detectar en la tierra debido a que las ondas submilimétricas son absorbidas por el vapor de agua presente en la atmósfera. Por ello, se han hecho aplicaciones espaciales para medir la radiación de $\mathrm{THz}$, o arreglos de antenas en la tierra en zonas muy secas como es el caso del Atacama Large Millimeter Array instalado en el desierto de Atakama en Chile [14].

A lo largo de este capítulo, se proporciona una breve descripción teórica de los principales generadores de $\mathrm{THz}$ artificiales, los cuales son clasificados en fuentes continuas o pulsadas según el principio físico involucrado [1], concluyendo con el utilizado en el presente trabajo. Asimismo, los detectores también son clasificados en dos grupos. En los casos en que se pueda medir la intensidad y fase del campo eléctrico se los agrupa en detectores coherentes, y en el caso en que se mida solo la intensidad en detectores no coherentes.

Cabe mencionar que muchas de las formas de generación y detección que serán detalladas, se han podido realizar gracias al desarrollo de los láseres modelocking que dieron la posibilidad de generar pulsos ultracortos de luz de subpicosegundos, hasta algunos femtosegundos (fs) [15]. 


\subsection{Generadores}

\subsubsection{Principales fuentes continuas de $\mathrm{THz}$}

\subsubsection{Generación por diferencia de frecuencias}

Mediante el mezclado de dos haces láseres continuos con diferentes frecuencias $\left(\omega_{1}, \omega_{2}\right)$, se forma un batido óptico que genera radiación de $\mathrm{THz}$ continua $\left(\omega_{1}-\omega_{2}\right)$, cuando la misma incide sobre un medio no lineal (Fig. 2.1). La generación por diferencia de frecuencias es un proceso no lineal de segundo orden. La conversión depende principalmente del coeficiente no lineal del material, y de las condiciones de coincidencia de la velocidad fase [16].

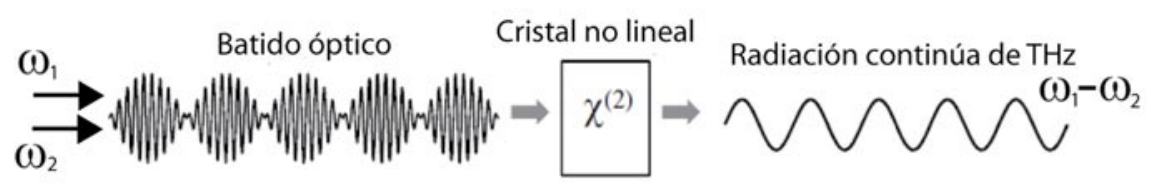

Figura 2.1: Generación por diferencia de frecuencias.

\subsubsection{Mezclado de fotones}

$\mathrm{Al}$ igual que en el caso anterior, se genera la radiación de $\mathrm{THz}$ mediante un batido óptico. Aunque en este caso, se incide sobre una antena fotoconductora (Fig. 2.2) [16]. La misma será descripta en las fuentes pulsadas.

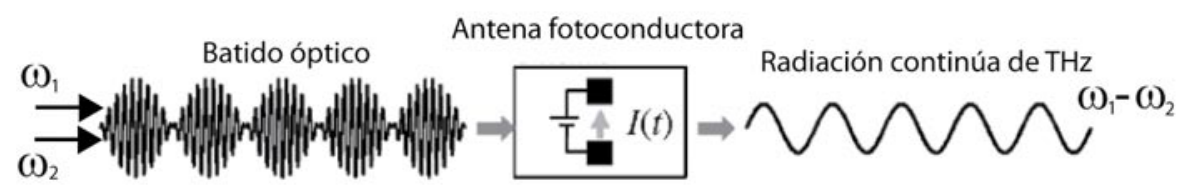

Figura 2.2: Mezclado de fotones.

\subsubsection{Multiplicación de frecuencias de microondas}

Mediante la incidencia de microondas sobre un diodo con características fuertes no lineales entre la tensión y corriente, se generan armónicos que alcanzan los THz (Fig. 2.3) [17]. 


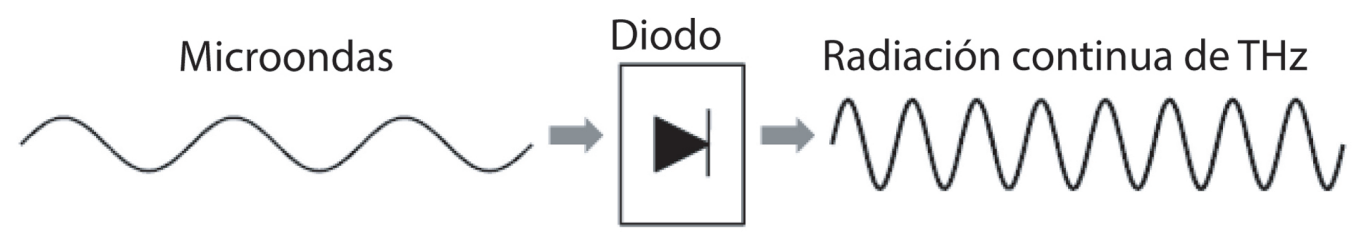

Figura 2.3: Multiplicación de frecuencias de microondas.

\subsubsection{Haz de electrones ondulados periódicamente}

En el caso de backward wave oscillators (BWO) y láseres de electrones libres (FELs)), el haz de electrones es modulado por una estructura periódica. En el caso del BWO, es una red de metal y en el FEL, un arreglo magnético. La radiación continua de $\mathrm{THz}$ es producida por la aceleración periódica de los electrones [1].

\subsubsection{Quantum cascade laser (QCL)}

Los niveles de energía de los pozos cuánticos en semiconductores pueden ser diseñados y fabricados con cierta facilidad para ser de cualquier valor. En tales sistemas, las transiciones entre sub-bandas que involucran electrones que generan las transiciones que provocan radiación dentro de las sub-bandas de la banda de conducción, pueden ser utilizadas para realizar emisiones en el infrarojo lejano y en las regiones de THz. Tales láseres son conocidos como QCL [16].

\subsubsection{Láser infrarrojo lejano (FIR)}

Los láseres infrarrojo lejano que logran llegar hasta frecuencias de $\mathrm{THz}$, surgen de la transición a un estado excitado vibracional de una molécula polar de gas a baja presión. El bombeo óptico del láser depende de la absorción selectiva de la radiación sintonizada del infrarrojo, de manera de lograr inversión de población entre los estados rotacionales generando radiación láser. En la práctica, se utiliza un número pequeño de moléculas para proveer frecuencias discretas en todo el espectro del FIR.

\subsubsection{Principales fuentes pulsadas de $\mathrm{THz}$}

\subsubsection{Rectificación óptica}

La radiación de $\mathrm{THz}$ debida al efecto de rectificación óptica, se genera debido al movimiento no lineal de los electrones en un cristal causados al incidir con 
un láser pulsado de femtosegundos con una intensidad tal que genere dicho fenómeno (Fig. 2.4).

Tal radiación, es consecuencia de la rectificación óptica del láser de fs, dado por la mezcla de diferencia de frecuencias que ocurren con las componentes del ancho de banda del láser dentro del material [2].

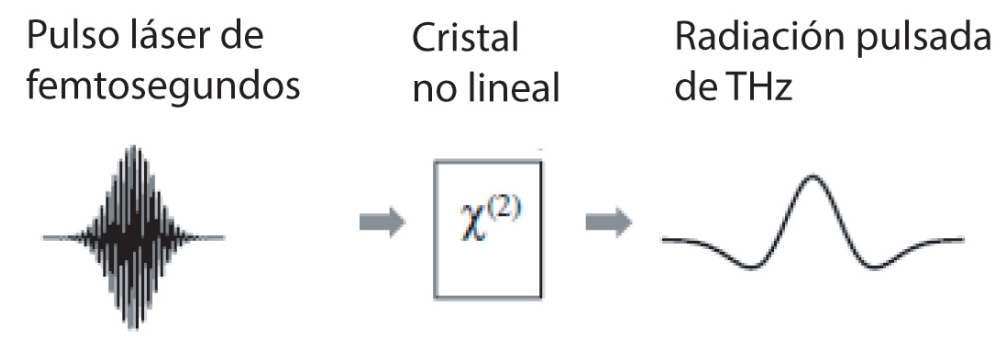

Figura 2.4: Rectificación óptica.

\subsubsection{Electrones relativistas acelerados (bunch of relativistic elec- trons )}

En este caso, un láser pulsado de femtosegundos dispara una fuente de electrones que genera un pulso ultracorto de electrones. Al acelerar al mismo a una velocidad relativista, e incidiéndolo contra un objetivo de metal, o al forzarlo a un movimiento circular por causa de un campo magnético, se produce radiación de $\mathrm{THz}$ coherente por aceleración transitoria de los electrones.

La mayor intensidad de $\mathrm{THz}$ lograda con el presente método, tuvo lugar en el Source Development Lab (SDL) en el Brookhaven National Laboratory, que pertenece al National Synchrotron Light Source (NSLS) [18].

\subsubsection{Plasma en aire inducido por láser}

La irradiación de $\mathrm{THz}$ mediante esta técnica, esta dada por la generación de plasma en aire causada por un pulso láser de femtosegundos de alta energía, junto con su segunda armónica [19] (Fig. 2.5).

Los $\mathrm{THz}$ son originados por la fotocorriente transitoria de los electrones ionizados, que son impulsados por la asimetría de la componente fundamental del campo eléctrico junto con su segunda armónica. Este fenómeno es descripto como un proceso de mezclado de cuatro ondas (four wave mixing), que es un proceso óptico no lineal de tercer orden [20]. 


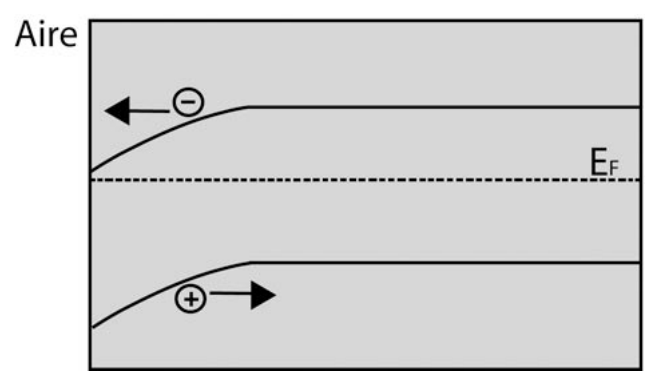

Figura 2.6: Esquema de bandas de un semiconductor cerca de la superficie.

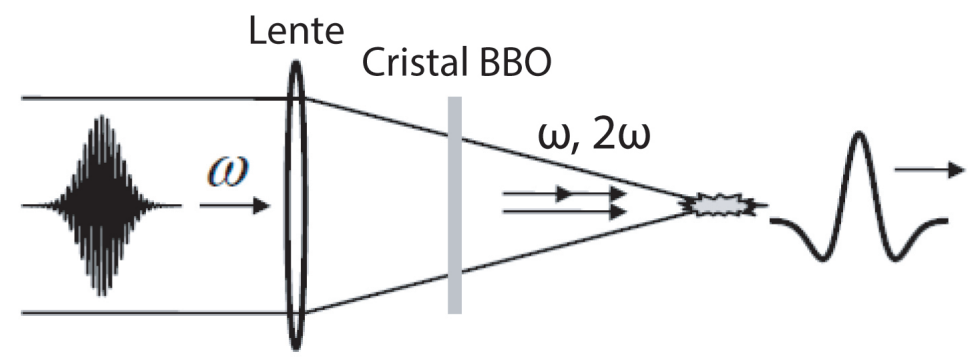

Figura 2.5: Plasma en aire inducido por láser.

\subsubsection{Conmutación transitoria fotoconductiva}

La radiación de $\mathrm{THz}$ es causada por un pulso temporal de corriente menor a un picosegundo que se genera mediante la incidencia de un láser de pulsos de femtosegundos, a una antena fotoconductora.

La misma, es un conmutador eléctrico que utiliza el incremento de la conductividad eléctrica de los semiconductores cuando son expuestos a la luz. La fotoconductividad resulta de un aumento del número de pares electrón-hueco generados por los fotones incidentes. Estos deben tener la energía suficiente para superar la banda prohibida del material [1].

Si el material semiconductor no esta polarizado (unbiased) por un campo eléctrico externo aplicado, los fotoportadores generados por el pulso láser son acelerados hacia la superficie. Esto ocurre, debido a que se genera un campo de vaciamiento a causa de que la interfase aire-semiconductor del material presenta una curvatura en la banda de valencia y de conducción, pero no así del nivel de Fermi (Fig. 2.6). Por tal motivo, los electrones y los huecos generados se trasladan en diferentes direcciones generando la fotocorriente responsable de la emisión de $\mathrm{THz}$ que se desvanece rápidamente debido al corto tiempo de vida media de los fotoportadores que se recombinan [21].

En el caso en que el material este polarizado (biased), el semiconductor 


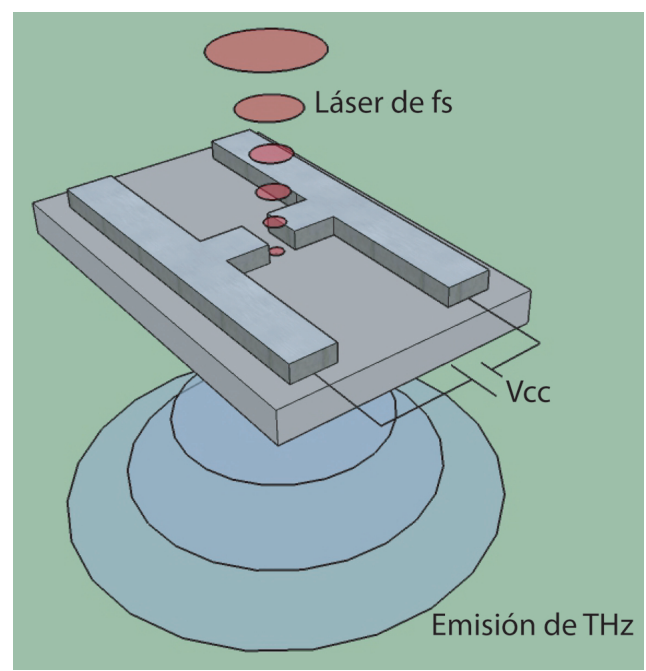

Figura 2.7: Esquema de la emisión de $\mathrm{THz}$ de una antena fotoconductora polarizada excitada por un láser de fs.

cuenta con dos electrodos metálicos en su superficie por los cuales se aplica una tensión (ver Fig. 2.7). La misma, genera un campo eléctrico que acelera a los fotoportadores provocados por el pulso láser causando la fotocorriente. Debido a la cantidad de defectos y trampas del material semiconductor se extingue en menos de un picosegundo generando radiación electromagnética que llega hasta frecuencias de THz. Dicha clase de antena es la mas utilizada ya que se logra mayor intensidad de radiación, y no dependen del ángulo de incidencia del haz láser en el semiconductor, como ocurre en el caso en el que el material no esta polarizado.

La radiación de $\mathrm{THz}$ puede describirse teóricamente mediante el uso de las ecuaciones de Maxwell, ya que se genera a través de un flujo de corriente. El modelo mas simple que se utiliza es el que asume a la antena como un dipolo eléctrico oscilante [1], aunque hay modelos mas complejos y certeros que son obtenidos de resolver las ecuaciones de Maxwell sobre la configuración particular de la antena [22]. Sin embargo, para lograr un modelo mas exacto, habría que recurrir a un tratamiento cuántico ya que el flujo de cargas que ocurre en el semiconductor sucede en tiempos de femtosegundos [23].

Utilizando el modelo descripto en la referencia [22] que obtiene una buena verificación experimental y es relativamente simple, se llega a que la conductividad en la superficie del material puede escribirse como:

$$
\sigma_{s}(t)=\frac{e(1-R)}{h v} \int_{-\infty}^{t} d t^{\prime} \mu\left(t-t^{\prime}\right) I_{\mathrm{opt}}\left(t^{\prime}\right) e^{-\frac{t-t^{\prime}}{\tau_{c}}},
$$




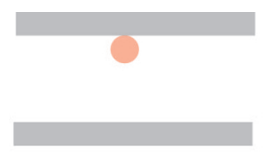

Stripline

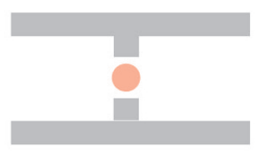

Dipole

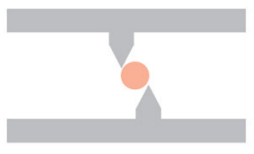

Ofsset dipole

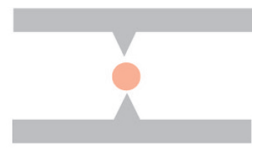

Bow tie

Figura 2.8: Configuraciones de electrodos de antenas fotoconductoras.

donde $R$ es la reflectividad óptica del área iluminada, $\mu(t)$ es la movilidad de los portadores, $I_{\mathrm{opt}}(t)$ es la intensidad del pulso láser, $h v$ es la energía de los fotones, y $\tau_{c}$ es el tiempo de vida media de los portadores.

$\mathrm{Al}$ resolver las ecuaciones de Maxwell teniendo en cuenta el tamaño finito del emisor, se obtiene que la amplitud del campo eléctrico irradiado es:

$$
E_{T H z}=-E_{\text {bias }} \frac{\sigma_{s}(t) Z_{0}}{\sigma_{s}(t) Z_{0}+1+n}
$$

donde $E_{\text {bias }}$ es el campo eléctrico aplicado, $Z_{0}=1 / \epsilon_{0} c=376,7 \Omega$ es la impedancia del espacio libre, y $n$ es el índice de refracción del semiconductor.

En la ecuación (2.2) se puede ver que la radiación de $\mathrm{THz}$ aumenta linealmente con la tensión de polarización. Tal afirmación es cierta mientras sea válida la ley de Ohm, ya que cuando la tensión de polarización es alta, la dependencia del campo con la conductividad tiene que tenerse en cuenta. También se puede ver que la radiación de $\mathrm{THz}$ aumenta, y luego se satura a medida que la intensidad del láser se incrementa. Esto es debido a que la conductividad es proporcional a la intensidad del láser.

Las antenas fotoconductoras se han clasificado dependiendo la forma de los electrodos metálicos utilizados. A través de las distintas configuraciones, se han conferido diferentes potencias o anchos de banda a las ondas generadas [1]. En la Figura 2.8 se presentan los casos stripline, dipole, offset dipole, y Bow-tie [24] que son los mas comunes.

En el caso de generación continua por mezclado de fotones, las antenas fotoconductoras presentan otras configuraciones de electrodos como es el caso Log-spiral, u otras mas modernas como el desarrollado en la referencia [25].

Cabe mencionar, que los materiales mas comunes utilizados como sustrato en las antenas son : Arseniuro de Galio crecido a baja temperatura (LT-GaAs), silicio en zafiro con daño por radiación (RD-SOS), arseniuro de galio dopado con cromo (Cr-GaAs), fosfuro de indio (InP), y silicio amorfo. Cada uno de ellos presenta como principal característica una vida media de los portadores por debajo del picosegundo.

En el trabajo experimental realizado, se utilizó como generador una antena fotoconductora de Arseniuro de Galio crecida a baja temperatura (LT-GaAs) 


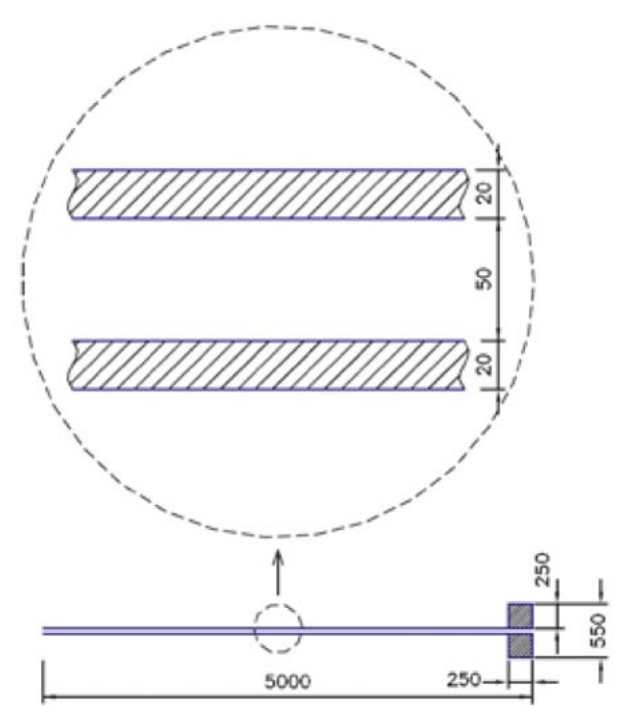

Figura 2.9: Antena generadora stripline (Marca Teravil).

(marca Teravil) con una configuración de los electrodos tipo stripline. En la Figura 2.9 se muestran las dimensiones de la antena en micrones.

\subsection{Detectores}

\subsubsection{Detección no coherente}

\subsubsection{Bolómetros}

Los bolómetros están equipados con una resistencia térmica que consiste en un semiconductor como Si, o Ge altamente dopado. En general, los bolómetros tienen una referencia a una temperatura criogénica que se compara con la variación de temperatura del sensor sensible a la radiación absorbida.

\subsubsection{Piroeléctricos}

Los sensores piroeléctricos cuentan con un sensor térmico que al absorber la radiación electromagnética, genera un cambio de temperatura que provoca una variación en la polarización eléctrica. 


\subsubsection{Celda de Golay}

En la celda de Golay, el sensor térmico cambia su temperatura y transfiere el calor a un gas que al expandirse ejerce una presión sobre una membrana. La cual, debido a sus cambios de forma al irradiarla con luz cambia la reflectividad óptica generando una medida sobre la radiación. Debido a que el sensor tiene que llegar a un equilibrio térmico, el tiempo de detección es relativamente lento en comparación con detectores típicos de luz.

\subsubsection{Detección coherente}

\subsubsection{Mezclado de fotones}

En este caso, al igual que en la emisión por mezclado de fotones, se irradia una antena fotoconductora con el mismo batido óptico utilizado para excitar al emisor. Como consecuencia, se generan fotoportadores que al ser acelerados por el campo eléctrico de $\mathrm{THz}$ proveniente del emisor, se produce una fotocorriente. La misma, cuenta con una dependencia relativa entre la fase del batido óptico, y la radiación de $\mathrm{THz}$ otorgando la información necesaria para obtener el campo eléctrico [26].

\subsubsection{Detección heterodina}

Este método se basa en mezclar la señal de $\mathrm{THz}$ de frecuencia $\omega_{s}$, con una frecuencia de referencia $\omega_{\text {ref }}$ de modo de provocar una señal de frecuencia intermedia que es la diferencia de ambas $\omega_{D}=\left|\omega_{\text {ref }}-\omega_{s}\right|$ (downconversion) [27]. Al reducir la frecuencia de la señal, se posibilita la medición a través de equipos electrónicos como analizadores de redes. Como mezcladores se utilizan comúnmente superconductores SIS y HEB .

El presente método es utilizado en diferentes observatorios como el ISO, SWAS, SOFIA, ALMA y Herschel [28].

\subsubsection{Detección por plasma}

Para detectar la radiación de $\mathrm{THz}$ producida por plasma en aire, se necesita implementar la configuración interferométrica que se presenta en la Fig. 2.10.

La medida de la amplitud del campo eléctrico es proporcional a la energía de la componente fundamental del pulso láser, y a la raíz cuadrada de la energía del pulso de segunda armónica. Los THz son obtenidos cuando todas las ondas 


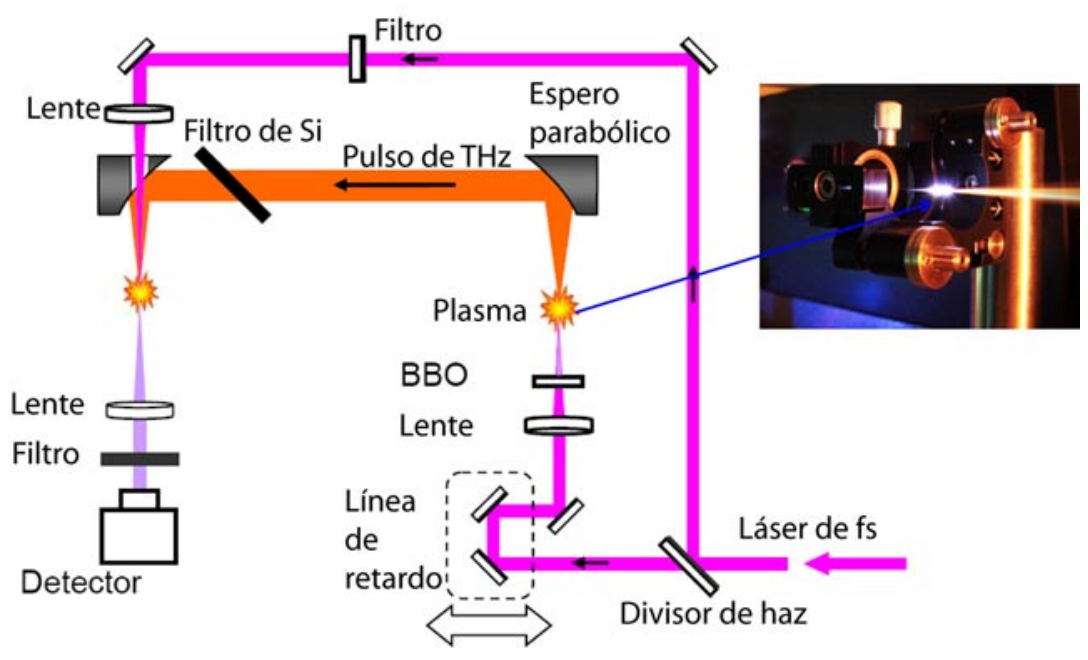

Figura 2.10: Configuración experimental para detección por plasma.

$(\omega, 2 \omega, \mathrm{y} \mathrm{THz})$ tienen la misma polarización en el proceso de mezclado de cuatro ondas [29].

\subsubsection{Muestreo electroóptico y conmutación fotoconductiva}

En ambas técnicas se utiliza un láser de femtosegundos, y una configuración interferométrica como la que se describe en la Fig. 2.11. En la misma, se puede ver que el haz láser se divide en 2 partes con el uso de un divisor de haz (beam splitter) irradiando con los mismos al detector (pulso de prueba), y al generador (pulso de bombeo). Cuando en el detector llegan simultáneamente la onda de $\mathrm{THz}$ generada y el pulso de luz, se obtiene un pulso de corriente proporcional al campo eléctrico. Variando la línea de retardo se logra un mapeo punto a punto del campo. Esta clase de configuración es la utilizada para realizar espectroscopía por trasmisión resuelta en el tiempo .

\section{Muestreo electroóptico}

La detección electroóptica se logra cuando la radiación de $\mathrm{THz}$ y el haz de prueba coinciden de manera copropagantes en un cristal electro-óptico (Fig. 2.12). Cuando ambos inciden sobre el cristal, se produce una modulación de fase en el haz de prueba inducida por el campo eléctrico perteneciente a la radiación de THz. Esto ocurre debido a que el cristal presenta el efecto Pockels que provoca birrenfringencia en el material dependiente de la amplitud del campo eléctrico al que esta sometido. Una vez que emerge el haz de prueba del cristal, se incide 


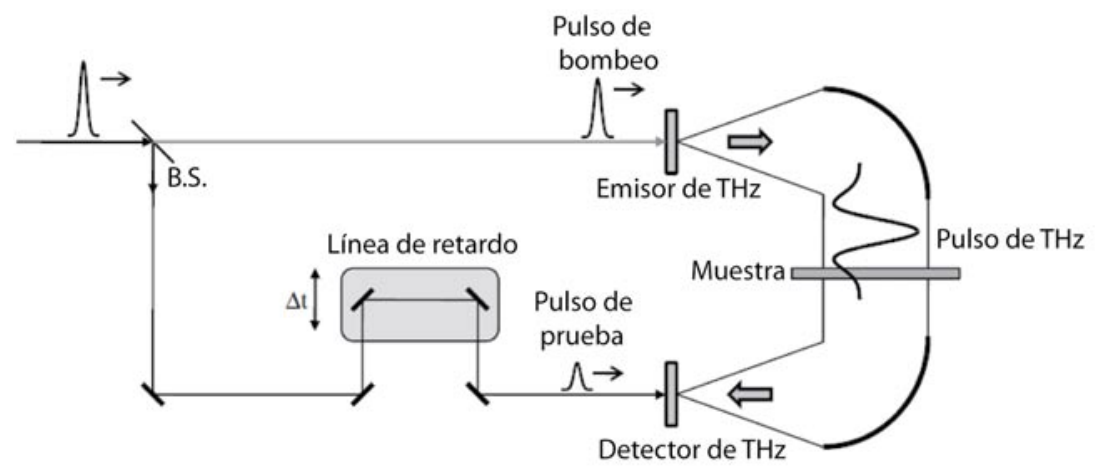

Figura 2.11: Configuración típica de un espectrómetro de THz.

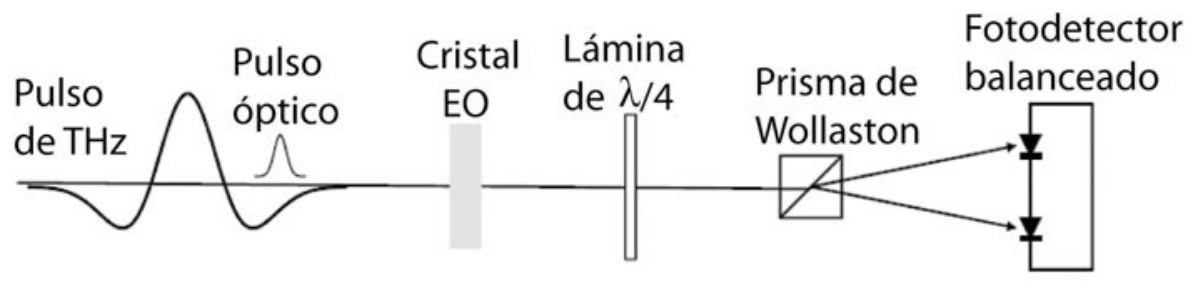

Figura 2.12: Configuración típica de detección por muestreo electro-óptico.

el mismo sobre una lámina de cuarto de onda y luego sobre un prisma de Wollaston que provoca que el haz sea divido en dos polarizaciones ortogonales que son finalmente detectadas por dos fotodiodos. Cabe mencionar, que luego del prisma de Wollaston la modulación de fase del pulso óptico se convierte en una modulación de intensidad en ambos haces emergentes.

En la Figura 2.13 se muestra cualitativamente el modo de detección cuando hay radiación de $\mathrm{THz}$ presente, como cuando no la hay. Se puede ver claramente en la figura que sólo se genera un desbalance en lo detectado en los fotodiodos, cuando hay radiación de $\mathrm{THz}$ presente.

\section{Conmutación fotoconductiva}

Al igual que en el caso de generación por conmutación transitoria fotoconductiva, se utilizan antenas fotoconductoras que cuentan con las mismas características físicas descriptas anteriormente. En este caso, dichas antenas tienen electrodos metálicos sobre el sustrato semiconductor, los cuales son utilizados para medir la corriente generada por causa de la radiación de $\mathrm{THz}$, y el pulso láser recibido. En este caso, la propia radiación de $\mathrm{THz}$ es responsable de polarizar los pares electrón-hueco generados en el material por el láser de fem- 


\section{Estado de polarización del pulso láser de prueba}

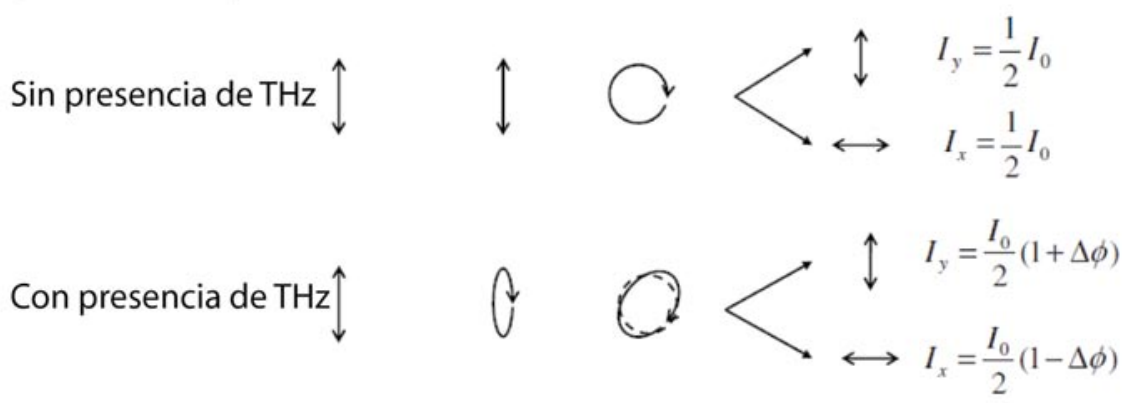

Figura 2.13: Principio de detección por muestro electro-óptico con y sin presencia de $\mathrm{THz}$.

tosegundos.

El proceso de detección se puede visualizar en la Fig. 2.14, donde se muestra que teniendo control del tiempo de arribo de la señal de THz, o del pulso de luz sobre la antena se puede hacer un mapeo del campo eléctrico (Fig. 2.14(d)).

Cuando el láser irradia el sustrato de la antena se genera un cambio temporal de la conductividad del material dado por $\sigma(t)=N(t) e \mu$, donde $N(t)$ es la densidad de cargas formada por la suma de huecos y electrones generados, y $\mu$ es la movilidad del material. Debido al tiempo de recombinación de los portadores, el material trabaja como una llave pasando de la conductividad a ser casi un aislante en menos de 1 ps. Con las dimensiones de la Fig. 2.15 y despreciando la resistencia provocada por los electrodos, podemos escribir a la resistencia de la antena como $R(t)=\omega / \sigma(t) A$, siendo $A \approx 2 d \alpha$ la sección transversal, con $\alpha$ la profundidad de absorción del semiconductor. La corriente generada por la ley de Ohm esta dada por $I(t)=V(t) / R(t)$, siendo la tensión de polarización $V(t) \cong E_{T H z}(t) h$.

Se puede observar en la Fig. 2.14(a) que el pulso de THz no llega simultáneamente con el pulso de luz en la antena, generando en la Fig. 2.14(d) que en el tiempo $t_{A}$ no haya señal detectada ya que $V(t)=0$. En los otros dos casos mostrados (Fig. 2.14(b,c)), se observa que el campo eléctrico coincide con el tiempo de arribo del pulso láser generando en cada posición un valor de corriente proporcional a la radiación de $\mathrm{THz}$, logrando el mapeo mostrado en la Fig. 2.14(d).

La respuesta temporal de la corriente medida en el detector, se puede estimar realizando la convolución del pulso de $\mathrm{THz}$ con la respuesta temporal de la antena que esta dada por $\sigma(t)$. Considerando que el pulso óptico arriba en 


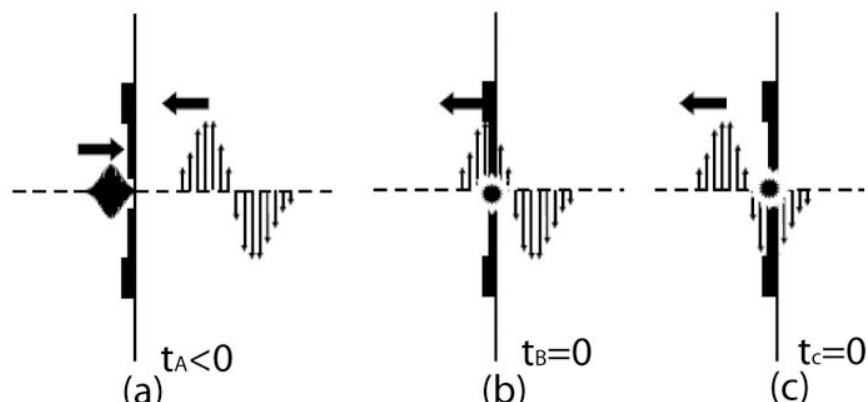

(a)

(b)

(c)

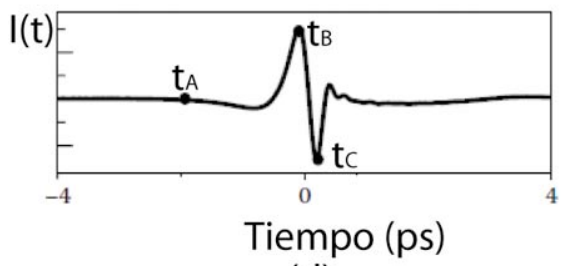

(d)

Figura 2.14: Mapeo de la detección del campo eléctrico.

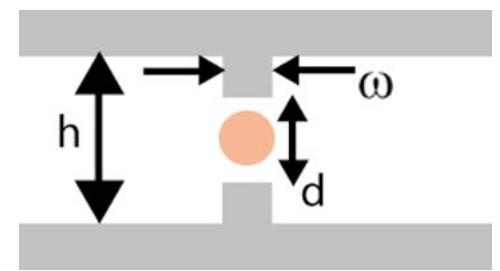

Figura 2.15: Dimensiones de antena fotoconductora. 
$t=0$, y el pulso de $\mathrm{THz}$ en $t=t_{0}$ la corriente se puede expresar como :

$$
I(t)=\frac{2 h \alpha}{w} \int_{-\infty}^{\infty} \sigma(t) E_{T H z}\left(t-t_{0}-\tau\right) d \tau
$$

En el límite en que la respuesta de la fotoconductividad sea mucho mas rápida que la duración del campo eléctrico, la corriente detectada es directamente proporcional al mismo. Cuando de manera opuesta, la fotoconductividad tiene un tiempo de vida mucho mayor que el campo eléctrico tenemos que $I(\omega)=E_{T H z}(\omega) / \omega$. Tomando el caso mas común que es cuando se presenta la situación intermedia, se obtiene [30] :

$$
I(\omega) \propto Q(\omega) P(\omega) E_{T H z}(\omega) .
$$

En (2.4) la corriente medida es el resultado de la convolución de la envolvente del pulso láser $(Q(\omega))$, con la respuesta de la antena $(P(\omega))$ y con el campo eléctrico de THz. Tal expresión, refleja la importancia que tienen tanto el tiempo de recombinación de los portadores, como el ancho temporal del pulso láser. Ya que cuánto más se aproximen ambos a una delta temporal, mas exacto será el resultado a la hora de obtener el campo eléctrico. También en (2.3), se destaca la importancia en las dimensiones de los electrodos de la antena, motivando la existencia de diferentes configuraciones.

En el trabajo experimental se utilizó para la detección una antena fotoconductora de Arseniuro de Galio crecida a baja temperatura (LT-GaAs) (marca Teravil), con la configuración que se muestra en la Fig. 2.16. La misma presenta las dimensiones de la antena en micrones. 


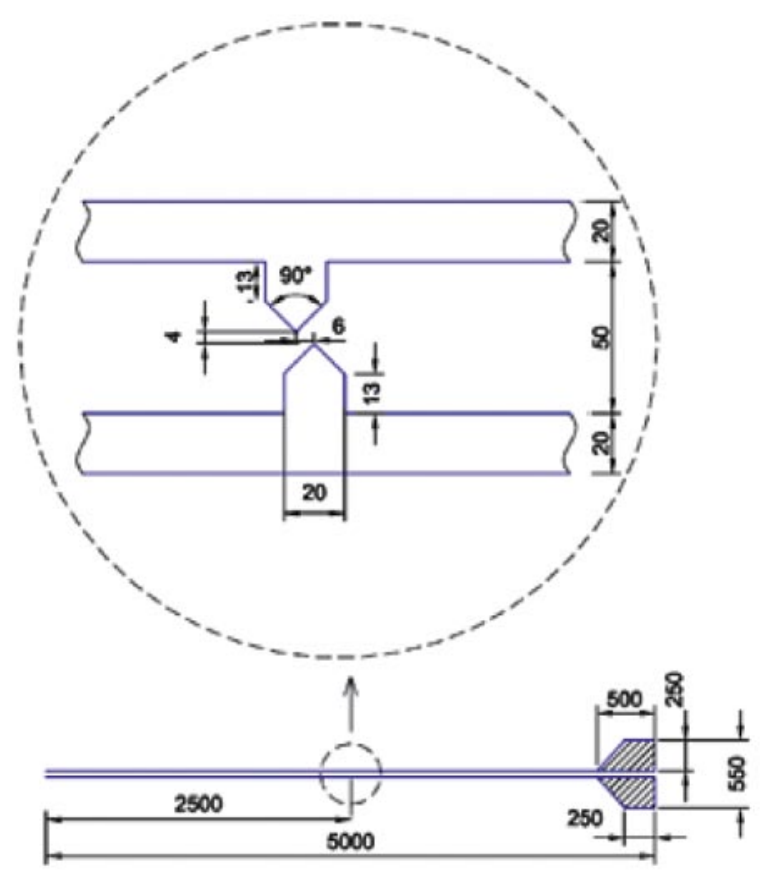

Figura 2.16: Antena detectora dipole offset (marca Teravil). 


\section{Capítulo 3}

\section{Desarrollo experimental de un espectrómetro de terahertz resuelto en el tiempo}

\section{Introducción}

La espectroscopía de $\mathrm{THz}$ fue desde el principio una de las principales aplicaciones desarrolladas en dicha banda. Ello no sólo ha sido importante para la espectroscopía en sí misma, sino que ha favorecido a otras áreas, como la obtención de imágenes y la astronomía [31, 32].

Las principales características de dicha banda que favoreció el creciente interés para desarrollar aplicaciones son:

-Los materiales dieléctricos como las telas, plásticos, maderas, cerámicos, etc, presentan baja absorción a la radiación de $\mathrm{THz}$, generando la posibilidad de caracterizar o estudiar dichos materiales en muchas aplicaciones como imágenes para seguridad, análisis de calidad en plásticos, madera, etc [33-35].

-Al ser la radiación de $\mathrm{THz}$ no ionizante genera ventajas frente a los análisis desarrollados con rayos X, aunque se encontró que altas intensidades generan daño al ADN de muestras de tejido humano [36].

-Ciertos materiales como los explosivos y drogas poseen frecuencias de absorción en la banda de THz que permiten su identificación [37].

La espectroscopía resuelta en el tiempo que utiliza fuentes pulsadas, se logra generalmente con el uso de la información obtenida al detectar dos pulsos elec- 


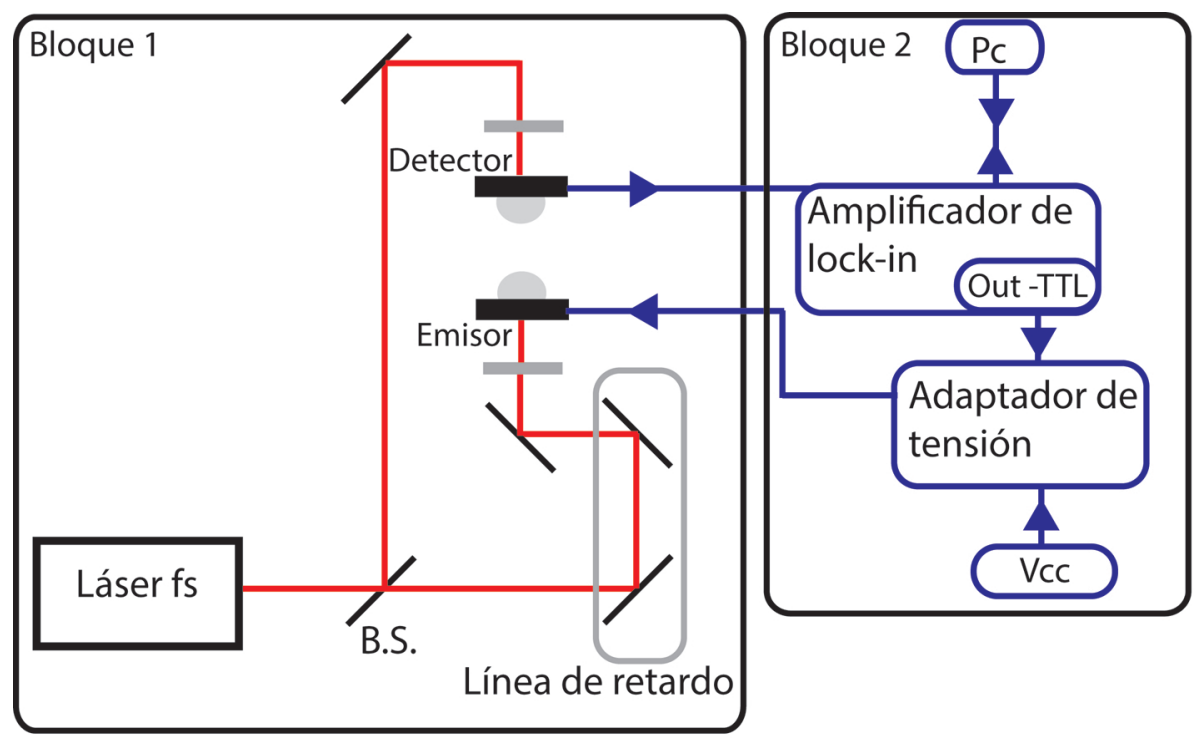

Figura 3.1: Configuración típica de un espectrómetro de THz.

tromagnéticos de subpicosegundos proveniente del generador. Uno se obtiene cuando se encuentra presente la muestra que se pretende caracterizar y el otro pulso, llamado referencia, se mide sin muestra alguna. Realizando básicamente el cociente de las transformadas de Fourier de ambos pulsos, se obtiene la función transferencia en el dominio de la frecuencia con la que se puede obtener los parámetros ópticos del material.

En la Figura 3.1 se muestra una configuración típica para los sistemas que cuentan con antenas fotoconductoras tanto para generar la radiación de $\mathrm{THz}$ como para detectarla [38]. En el presente trabajo se utilizó dicha configuración con la propagación del haz láser en espacio libre, aunque en algunos casos la propagación se realiza mediante fibra óptica. En el Bloque 1, se puede ver al láser con la configuración interferométrica tal como fue descripta en la Fig. 2.11 de la Sección 2.2. En el Bloque 2 se encuentra los componentes restantes necesarios para la detección y generación de la señal. Debido a que en el detector la corriente puede llegar a ser del orden del picoampere, se utiliza un amplificador de lock-in. Para alternar la señal se varía la tensión del generador en sincronismo con la referencia del lock-in. Los datos adquiridos del amplificador son transferidos mediante un software de adquisición a la PC.

En las subsecciones siguientes será descripta cada una de las partes de los bloques. Las mismas han sido desarrolladas a lo largo de la tesis. Finalmente se concluye el presente capítulo con los primeros resultados obtenidos del espectrómetro. 


\subsection{Interferómetro de Michelson}

Para la realización del espectrómetro primeramente se montó en la mesa óptica el interferómetro de Michelson de manera que los haces láser de cada brazo del mismo resultaran en una configuración contrapropagante (Bloque 1 de la Fig. 3.1).

Para ello se diseñaron y fabricaron las piezas mecánicas necesarias para todas las monturas de los espejos, divisor de haz, línea de retardo, etc. De esta manera se logró que los haces tengan la misma altura a lo largo de la mesa óptica obteniendo así una correcta alineación. Tanto los espejos, divisor de haz, como los demás elementos utilizados, eran adecuados para trabajar con el láser de femtosegundo. Mediante el uso del equipo FROG (frequency resolved optical gating) de Swamp Optics, se verificó que las reflexiones como la trasmisiones en los mismos, no generaran dispersión en el pulso láser.

Para poder ubicar correctamente la posición de la antena detectora, de manera que la radiación de THz y el pulso láser lleguen simultáneamente, se buscó primeramente encontrar la coincidencia de los pulsos contrapropagantes sin las antenas presentes. Como resultado accesorio de la experiencia, se mostró que se podían utilizar los resultados para medir el ancho temporal del pulso [10]. De todas maneras, el pulso láser había sido previamente caracterizado mediante el uso del equipo comercial FROG. Caracterizar al mismo, es de vital importancia para posibilitar la correcta generación y detección de radiación de THz. Más aún, las antenas fotoconductoras especifican el ancho máximo temporal que puede tener el haz láser para su correcto funcionamiento $(\leq 150 \mathrm{fs})$.

Luego de encontrar la coincidencia de los pulsos contrapropagantes, se colocaron las antenas fotoconductoras y otros elementos (lámina retardadora, polarizador, lentes, etc) en la mesa óptica con ciertas consideraciones detalladas en el presente capítulo. Lográndose así, una correcta generación y detección de señal.

\subsubsection{Caracterización del láser de pulsos ultracortos de luz}

\section{Resumen}

El método propuesto para caracterizar el pulso láser se basa en la detección de la fluorescencia causada por absorción de dos fotones (TPA). La misma es emitida por una celda con Rhodamina B en ethanol en la posición de encuentro de los pulsos contrapropagantes. Con los resultados obtenidos y mediante un desarrollo teórico, se encontró que el ancho temporal a mitad de altura (FWHM) 
es $F W H M_{\text {medido }}=F W H M_{\text {pulso-simple }} / \sqrt{2}$. A diferencia del resultado más general dado por $F W H M_{\text {medido }}=F W H M_{\text {pulso-simple }} \sqrt{2}$, que es el caso donde los pulsos tienen una configuración copropagante. Se define $F W H M_{\text {pulso-simple }}$ como el ancho a mitad de altura del láser y a $F W H M_{\text {medido }}$ como al ancho a mitad de altura de la imagen resultante de la superposición de los dos pulsos contrapropagantes del láser. También se hizo un análisis de la influencia del chirp, y se propuso un método de calibración.

\subsubsection{Introducción}

Junto con el avance tecnológico de los láseres han avanzado los métodos para caracterizarlos. Se destacan mayormente el de autocorrelación de intensidad [39, 40], autocorrelación de tercer orden [41] y en particular uno de los más novedosos llamado FROG [42]. A continuación se describen dos técnicas que se fundamentan en la autocorrelación empleando efectos no lineales:

\section{Autocorrelación de segundo orden}

Se fundamenta en la utilización del fenómeno conocido como generación de segunda armónica (SHG) $[43,44]$. La misma se logra a través del uso de un medio que tenga alta susceptibilidad no lineal de segundo orden [45]. Para poder medir el pulso se requiere obtener sincronismo de fase y un detector que es típicamente un fotomultiplicador.

\section{Absorción de dos fotones (TPA)}

Este método se basa en observar la fluorescencia emitida debido al decaimiento de los fotones desde un cierto nivel de energía. Este se consigue por la absorción de dos fotones coherentes como consecuencia de un proceso no lineal de tercer orden [43] (Fig. 3.2).

TPA ha dado la posibilidad de construir sistemas de autocorrelación [46], correlación cruzada [47], realizar mediciones de pulsos ultracortos de luz, e incluso desarrollar aplicaciones en comunicaciones ópticas [48].

En la referencia [49] se puede ver una revisión de los diferentes métodos desarrollados a lo largo de los años para medir pulsos de luz mediante el uso de TPA. Entre ellos se muestran algunos esquemas basados en análisis temporales o espaciales [50-52], los cuales retrasando un brazo del interferómetro, se obtiene una traza espacial o temporal mediante la detección de la fluorescencia utilizando densitómetros, fotomultiplicadores, etc.

En esta tesis se mide el ancho temporal del pulso láser mediante la realización de un interferómetro de Michelson en una configuración contrapropagante, y se 


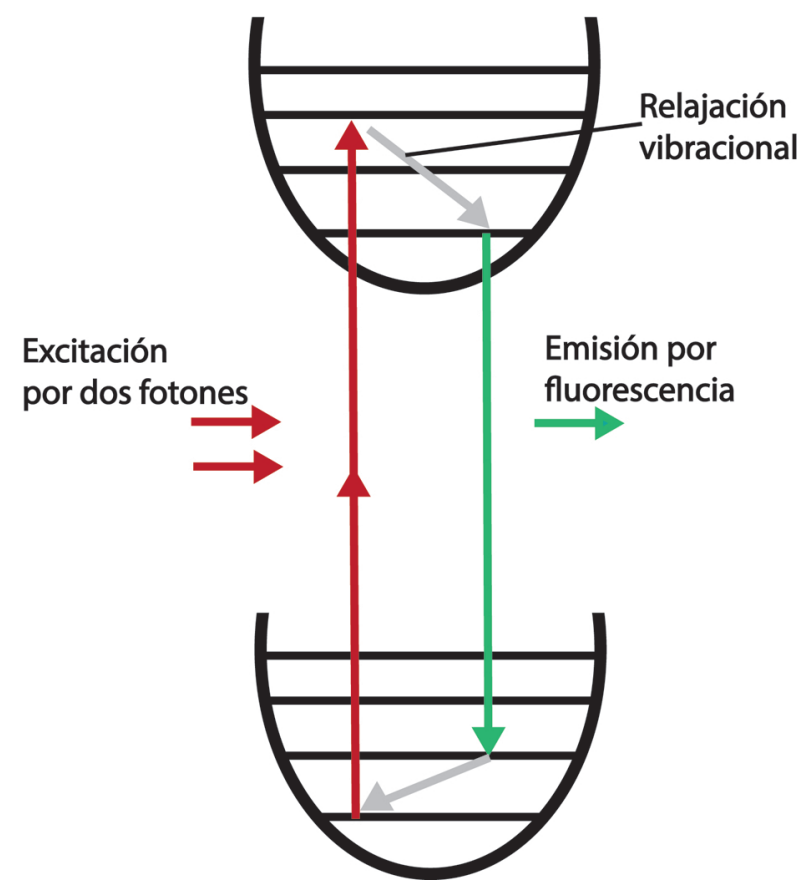

Figura 3.2: Esquema de emisión de radiación debido a TPA.

utiliza la emisión fluorescente luego de la TPA. La emisión se registra en una cámara fotográfica estándar.

\subsubsection{Teoría}

A lo largo de esta Sección se realiza un análisis teórico para la configuración propuesta. En la misma, los pulsos se superponen de manera contrapropagante en una celda con Rhodamina B en ethanol (Fig. 3.3). A continuación se obtiene la fluorescencia emitida de la celda que es la responsable de proveer información sobre el ancho de los pulsos.

La intensidad disipada causada por la absorción de dos fotones en la celda con Rhodamina B en ethanol se puede escribir como [43] :

$$
\frac{\partial I_{\mathrm{int}}}{\partial x}=\beta\left|E_{0}\right|^{4}
$$

donde $E_{0}$ es el campo eléctrico, $\beta=\frac{3}{2 \epsilon_{0} n^{2} c^{2}} \omega \chi_{x x x}, \chi$ es la susceptibilidad, $\omega$ es la frecuencia angular, $n$ es el índice de refracción, $c$ es la velocidad de la luz y $\epsilon_{0}$ es la permitividad del vacío.

El campo eléctrico de dos pulsos en una configuración contrapropagante en dirección x puede escribirse como [52] : 


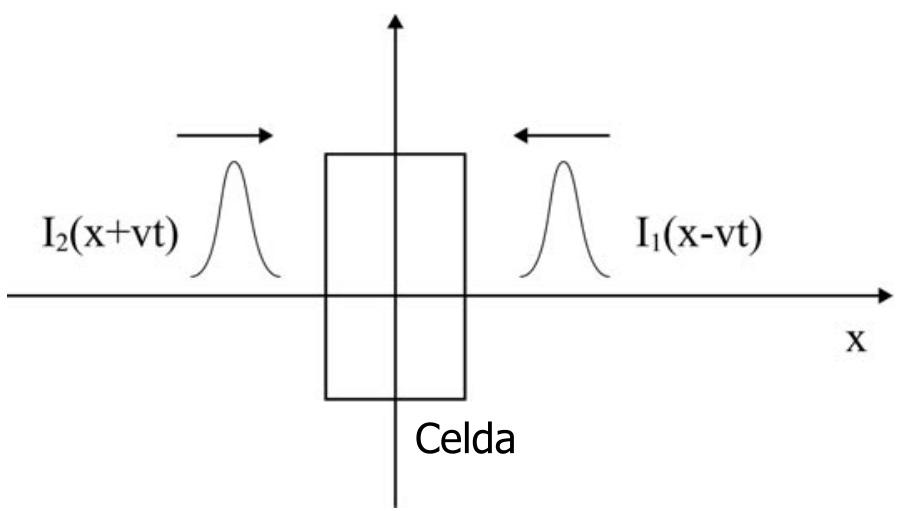

Figura 3.3: Configuración contrapropagante de los pulsos láser.

$$
E_{0}(y, z, x-v t)=E_{1}(y, z, x-v t) e^{-i\left(k x+\theta_{1}\right)}+E_{2}(y, z, x+v t) e^{i\left(k x+\theta_{2}\right)},
$$

siendo $v$ la velocidad de propagación del campo eléctrico y $k$ el vector de onda. Sustituyendo (3.2) en (3.1):

$$
\begin{aligned}
\frac{\partial I_{\text {int }}}{\partial x}(y, z, x-v t)= & \beta\left(E_{1}^{4}(y, z, x-v t)+E_{2}^{4}(y, z, x+v t)\right. \\
& +4 E_{1}^{3}(y, z, x-v t) E_{2}(y, z, x+v t) \cos \left(2 k x+\theta_{2}-\theta_{1}\right) \\
& +4 E_{1}(y, z, x-v t) E_{2}^{3}(y, z, x+v t) \cos \left(2 k x+\theta_{2}-\theta_{1}\right) \\
& +4 E_{1}^{2}(y, z, x-v t) E_{2}^{2}(y, z, x+v t) \\
& +2 E_{1}^{2}(y, z, x-v t) E_{2}^{2}(y, z, x+v t) \cos \left(4 k x+2\left(\theta_{2}-\theta_{1}\right)\right) .
\end{aligned}
$$

El tercer, cuarto y último término de (3.3) presentan una modulación espacial que no puede ser resuelta por una cámara digital estándar. Por tal motivo (3.3) se puede escribir como:

$$
\begin{aligned}
\frac{\partial I_{\text {int }}}{\partial x}(y, z, x-v t)= & \beta\left(E_{1}^{4}(y, z, x-v t)+E_{2}^{4}(y, z, x+v t)\right. \\
& +4 E_{1}^{2}(y, z, x-v t) E_{2}^{2}(y, z, x+v t) .
\end{aligned}
$$

La relación de la intensidad con el campo eléctrico se puede expresar de la siguiente manera:

$$
I_{\text {int }}=\frac{\epsilon_{0} c n}{2}\left|E_{0}\right|^{2}
$$


Reemplazando (3.5) en (3.4) se obtiene una expresión en función de las intensidades:

$$
\begin{aligned}
\frac{\partial \operatorname{Iint}}{\partial x}(y, z, x-v t)= & \gamma\left(I_{1}^{2}(y, z, x-v t)+I_{2}^{2}(y, z, x+v t)\right. \\
& +4 I_{1}(y, z, x-v t) I_{2}(y, z, x+v t)
\end{aligned}
$$

Integrando sobre las coordenadas de sección transversal $z, y$ y en el tiempo en (3.6), se obtiene el resultado de la solución de la traza espacial. Asumiendo que $I_{1}$ e $I_{2}$ son pulsos Gaussianos unidimensionales propagándose en la dirección del eje $\mathrm{x}$, con las mismas amplitudes y direcciones opuestas se obtiene:

$$
\begin{aligned}
& I_{1}=e^{-(x-v t)^{2}} . \\
& I_{2}=e^{-(x+v t)^{2}} .
\end{aligned}
$$

Mediante la sustitución de (3.8) y (3.7) en (3.6) e integrando la expresión resultante en el tiempo se consigue:

$$
\begin{gathered}
\int_{-\infty}^{\infty} I_{2}^{2} d t=\int_{-\infty}^{\infty} I_{1}^{2} d t=\int_{-\infty}^{\infty} e^{-2(x-v t)^{2}} d t=\frac{\sqrt{\pi}}{v \sqrt{2}} \\
\int_{-\infty}^{\infty} I_{1} I_{2} d t=\int_{-\infty}^{\infty} e^{-2(x-v t)^{2}} e^{-2(x+v t)^{2}} d t=4 e^{-2 x^{2}} \int_{-\infty}^{\infty} e^{-2(v t)^{2}} d t=\frac{4 \sqrt{\pi} e^{-2 x^{2}}}{\sqrt{2} v} .
\end{gathered}
$$

Con los resultados de las ecuaciones (3.9) y (3.10), se obtiene como traza espacial:

$$
I_{\mathrm{int}}(x)=\gamma\left(\frac{2}{v} \sqrt{\frac{\pi}{2}}+\frac{4 \sqrt{\pi} e^{-2 x^{2}}}{\sqrt{2} v}\right)
$$

A partir de (3.11) se observa que el segundo término resulta ser una función Gaussiana con una varianza menor a la del pulso simple. También se observa que el primer término tiene un valor constante, el cual es causado por la interacción independiente de cada pulso con la solución. El ancho medio a mitad de altura resultante de la expresión es:

$$
F W H M_{\text {medido }}=F W H M_{\text {pulso-simple }} / \sqrt{2} \text {. }
$$
[51]:

El contraste obtenido cuando ambos pulsos tienen la misma amplitud es 

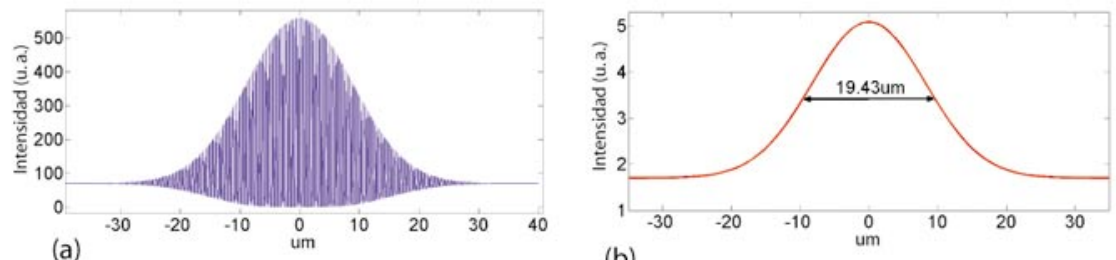

(b)

Figura 3.4: (a) Resultado de simular la ecuación (3.15). (b) Resultado de la convolución de la Fig. 3.4(a) con una función Gaussiana de $F W H M=1,145 \mu \mathrm{m}$ (resolución de la cámara).

$$
\frac{I_{\text {int }}(0)}{I_{\text {int }}(\infty)}=3
$$

El factor que relaciona los anchos a mitad de altura en (3.12) puede cambiar dependiendo la forma del pulso. Si se considera que el pulso láser puede ser aproximado por una función $\operatorname{sech}^{2}$ el factor de multiplicación sería 1.54 y no $\sqrt{2}$.

\subsubsection{Simulación Numérica}

Se realizó una simulación numérica utilizando la ecuación (3.1). Se asumieron dos pulsos Gaussianos de misma amplitud propagándose en la dirección x, con chirp lineal. Con tales consideraciones (3.2) se convierte en:

$E_{0}(x, t)=e^{\frac{-2 \ln 2}{a^{2}}(x-v t)^{2}} \operatorname{sen}\left(n k x-\left(\omega t+\pi b t^{2}\right)\right)+e^{\frac{-2 \ln 2}{a^{2}}(x+v t)^{2}} \operatorname{sen}\left(n k x+\omega t+\pi b t^{2}\right)$.

Se consideró $c=0,3 \mu \mathrm{m} / \mathrm{fs}, n_{\text {ethanol }}=1,361, \lambda_{\text {laser }}=0,8 \mu m, b=0, k=2 \pi / \lambda$, $\omega=c k, a=(c / n) \mathrm{T}, \mathrm{T}=123,8 \mathrm{fs}, \mathrm{y} v=c / n$.

La intensidad espacial causada por la absorción de dos fotones se obtuvo mediante la simulación de la siguiente expresión:

$$
I_{\mathrm{int}}(x)=\int_{-\infty}^{\infty}\left(E_{0}(x, t)\right)^{4} d t
$$

En la Figura 3.4(a) se observa el campo eléctrico obtenido que representa la radiación emitida por el solapamiento de los pulsos. La resolución de la cámara no puede detectar las oscilaciones de alta frecuencia. Entonces, para obtener un resultado más realista, se realizó la convolución entre $I_{\text {int }}(x)$ y un pulso 
Gaussiano de ancho medio a mitad de altura igual a la resolución de la cámara utilizada en el experimento $(2,29 \mu \mathrm{m})$. Obteniendo así lo mostrado en la Fig. 3.4(b).

Aproximando la curva obtenida en dicha figura por una función Gaussiana, se obtuvo un ancho medio espacial de $F W H M=19,43 \mu \mathrm{m}$, que resulta en el dominio temporal en 124,65 fs. Como se expuso en el marco teórico, el contraste obtenido en la figura entre el pico de la señal y el fondo es tres. También se comprobó que al mejorar la resolución de la cámara el resultado del ancho espacial del pulso se acerca al valor teórico $(F W H M=19,31 \mu \mathrm{m})$.

El chirp de los pulsos se simuló haciendo $b \neq 0$ en (3.14). El efecto del chirp en el autocorrelador se analizó utilizando como frecuencia instantánea $f(t)=$ $f_{0}-50 t 10^{-6} / \mathrm{fs}^{2}$. Ésta fue obtenida experimentalmente mediante la utilización de un equipo comercial que realiza la técnica FROG. El resultado en la simulación mostró que aún incrementando el chirp en un orden de magnitud no se observan cambios en el resultado.

\subsubsection{Experimento}

El experimento fue llevado a cabo mediante el uso de la configuración detallada en la Fig. 3.5. En la misma se puede distinguir el láser (MAI TAI, Spectra Physics) que cuenta con las características mostradas en la Fig. 3.6, el interferómetro con la línea de retardo y la celda con Rhodamina B en la zona del encuentro de los pulsos.

Se encontraron dos dificultades para lograr la correcta alineación de ambos brazos del interferómetro. Una fue lograr que los haces tengan la misma altura y otra fue que en el momento del encuentro tengan la misma dirección. Para prevenir dichos problemas se utilizaron agujeros de referencia a lo largo de la propagación de los haces.

Otra dificultad necesaria de resolver fue ubicar el punto de encuentro de los pulsos, ya que un pulso temporal de 100 fs espacialmente equivale a $30 \mu \mathrm{m}$. Por tal motivo, se utilizó una cinta métrica para ubicar la celda de manera equidistante a ambos brazos del interferómetro. Una vez ubicada la celda se realizó el ajuste fino mediante el uso de la línea de retardo. La misma, consistía en el uso de un motor paso a paso modelo DMX UMD de la empresa Arcus Technology acoplado a un trasladador marca K2CNC que cuenta con un paso de 0.1" por vuelta. Las características del motor se muestran en la Fig, 3.7. El mínimo paso de la línea de retardo controlado mediante encoder fue de $0.635 \mu \mathrm{m}$.

Al ver las imágenes obtenidas con la cámara de fotos (Sony DSC-H9) colocada frente a la celda se pudo evidenciar scattering. El mismo era provocado por la interacción de las partículas de polvo en la solución con los haces láser. Dicho efecto no permitía visualizar los cambios de contraste de luz en la celda, 


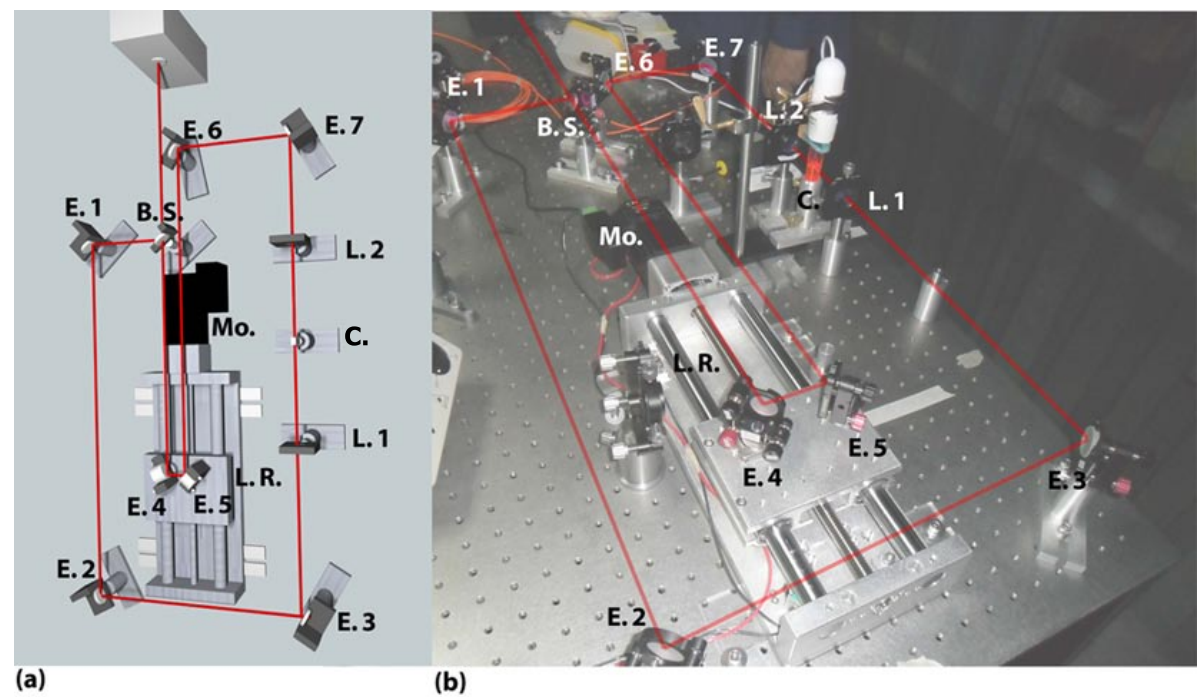

(a)

(b)

Figura 3.5: Configuración del espectrómetro de Michelson montado; E.1, E.2, E.3, E.4, E.5, E.6, E.7 son los espejos; B.S. es el beam splitter; L.1, L. 2 son las lentes de $5 \mathrm{~cm}$ de distancia focal; L.R. es la línea de retardo, Mo. es el motor paso a paso; C. es la celda de Rhodamina B donde los pulsos se encuentran. (a) Esquema gráfico. (b) Montaje experimental.

necesarios para detectar la fluorescencia producida por TPA (Fig. 3.8).

Como la fluorescencia producida por la Rhodamina B se encuentra cerca de $600 \mathrm{~nm}$ [53], se colocó un filtro de la empresa Noir Laser Shield modelo YG333, para eliminar las longitudes de onda alrededor de los $800 \mathrm{~nm}$ que eran las responsables de dicho scattering.

En la Figura 3.9 se muestran 2 imágenes obtenidas luego filtrar los $800 \mathrm{~nm}$ que muestran la emisión debida al encuentro de los pulsos en dos posiciones diferentes.

\subsubsection{Resultados}

Para obtener una buena calidad de imagen y aumentar la relación señal ruido, se realizó un promedio espacial del rectángulo mostrado en la Fig. 3.9. Obteniendo así, la Fig. 3.10 como la suma de ambas imágenes.

El contraste entre el pico y el fondo de la señal es 1.2 y no 3 como se expuso en la teoría, ya que los brazos del interferómetro no están perfectamente superpuestos en la celda y presentan diferentes intensidades.

Para conocer la relación pixel-longitud de la imagen obtenida se realizó un método de calibración que se puede dividir en dos partes. La primera consiste en tomar una foto en una determinada posición del encuentro de los pulsos (Fig. 


\section{Mai Tai ${ }^{\oplus}$}

\section{Specifications}

Output Characteristics

\begin{tabular}{lc} 
Pulse Width & $<100 \mathrm{fs}$ \\
\hline Tuning Range & $690-1040 \mathrm{~nm}$ \\
\hline Average Power at $800 \mathrm{~nm}$ & $<1 \mathrm{~W}$ \\
\hline Beam Roundness & $0.9-1.1$ \\
\hline Astigmatism & $<10 \%$ \\
\hline Repetition Rate & $80 \mathrm{MHz} \pm 1 \mathrm{MHz}$ \\
\hline Beam Pointing Stability & $<50 \mu \mathrm{rad} / 100 \mathrm{~nm}$ \\
\hline Noise & $<0.15 \%$ \\
\hline Stability & $< \pm 1 \%$ \\
\hline Spatial Mode & TEM \\
\hline Polarization & $\mathrm{M}^{2}<1.1$ \\
\hline Beam Divergence & $>500: 1 \mathrm{Horizontal}$ \\
\hline Beam Diameter $\left(1 / \mathrm{e}^{2}\right)$ & $<1.2 \mathrm{mrad}$ \\
\hline
\end{tabular}

Figura 3.6: Características del láser Mai Tai (Spectra Physics). 
- USB 2.0 communication

- RS-485 ASCII communication at 9600, 19200, 38400, 57600, 115200 bps

- Digital IO communication (opto-isolated inputs and outputs):

- 4 bit motion profile select inputs

- One start motion input

- One abort/clear motion input

- One in position output

- One error output

- $\mathrm{A} / \mathrm{B} / \mathrm{Z}$ Differential Encoder inputs

- Opto-isolated +Limit/-Limit/Home inputs

- Opto-isolated high speed position capture Latch input

- $1 \mathrm{M}$ pulse/second controller output

- 1000 line incremental encoder (4000 counts/rev with 4x quadrature decoding)

- StepNLoop closed-loop control

- S-curve or trapezoidal acceleration profile control

- On-the-fly speed change

- Two 10-bit analog inputs

- Joystick control

- Stand-alone programmable

- Homing routine using:

- Home input only

- Z index encoder channel only

- Home and $\mathrm{Z}$ index encoder channel

- 2-500 micro-step driver with effective resolution with 1.8 degree motor of up to $100,000 \mathrm{pulse} / \mathrm{rev}$

- 12 to 48VDC Voltage Input

- Driver current from $100 \mathrm{~mA}$ to 3.0A (peak current)

- NEMA 17/23 motor sizes available in different stack sizes.

Figura 3.7: Características del motor paso a paso DMX UMD.

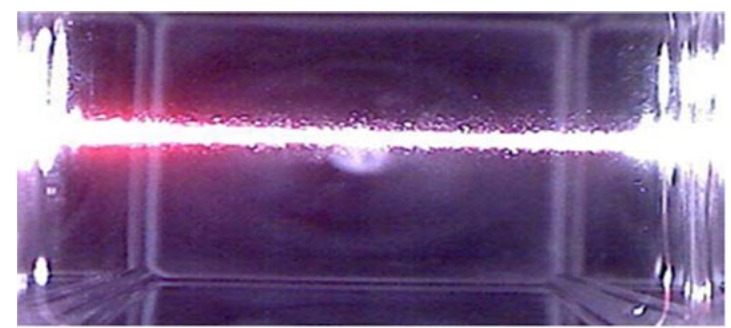

Figura 3.8: Fotografía con presencia de scattering. 


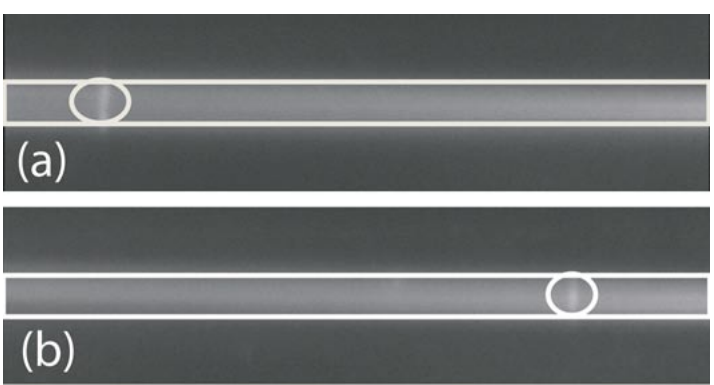

Figura 3.9: (a,b) Imágenes de los pulsos solapadados en distintas posiciones.

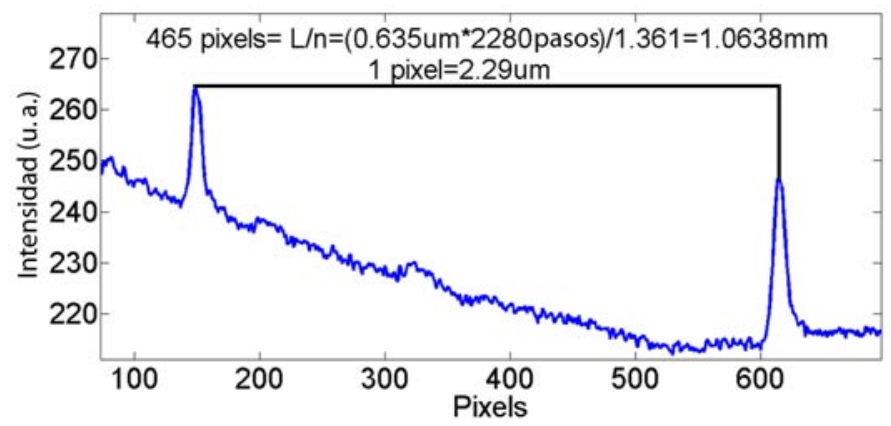

Figura 3.10: Promedio espacial de la suma de las dos imágenes de la Fig. 3.9. 


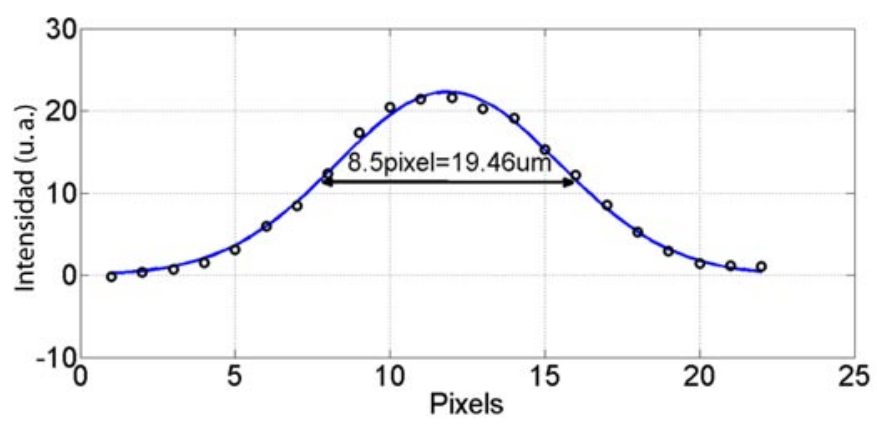

Figura 3.11: Aproximación de la emisión de fluorescencia de la Fig. 3.9(a) por una función Gaussiana.

3.9(a)). La segunda, en obtener una foto luego de mover la línea de retardo un número de pasos equivalentes a una longitud $L$ conocida, lo cual provoca la traslación del encuentro de los pulsos (Fig. 3.9(b)). Se obtiene la relación pixel-longitud y la resolución de la cámara teniendo en cuenta que el número de pixels encontrados en el desplazamiento del pico de la señal esta dado por $L / n_{\text {ethanol }}$ (ver Fig. 3.10).

Realizando una aproximación de la fluorescencia de la Fig. 3.9(a) por una función Gaussiana, el ancho medio a mitad de altura fue de $19.46 \mu \mathrm{m}$ (Fig. 3.11). Mediante el uso de la ecuación (3.12) se obtuvo que $F W H M_{\text {Pulso-simple }}=$ $F W H M_{\text {medido }} \sqrt{2} n / c=124,85$ fs. Para validar los resultados, el pulso láser se midió utilizando el instrumento comercial FROG que obtuvo FWHM= $123,8 \pm 0,02$ fs (Fig. 3.12), resultando en un valor similar al encontrado por el método propuesto en esta tesis.

El error cometido en la medida fue debido a la resolución de la cámara fotográfica que en este experimento fue de $\pm 1,145 \mu \mathrm{m} \equiv \pm 5,2$ fs.

\subsubsection{Conclusiones}

Se desarrolló un método novedoso para medir el ancho temporal de un láser de pulsos ultracortos de luz, demostrándose teórica y experimentalmente los resultados obtenidos. Asimismo se analizaron los errores involucrados y la influencia del chirp del láser.

\subsubsection{Montaje del interferómetro}

Para finalizar el montaje del interferómetro como se muestra en la Fig. 3.13, se adicionaron al sistema de la Fig. 3.5, las antenas fotoconductoras, lentes de enfoque, un atenuador, un telescopio de Galileo, una lámina retardadora y un 


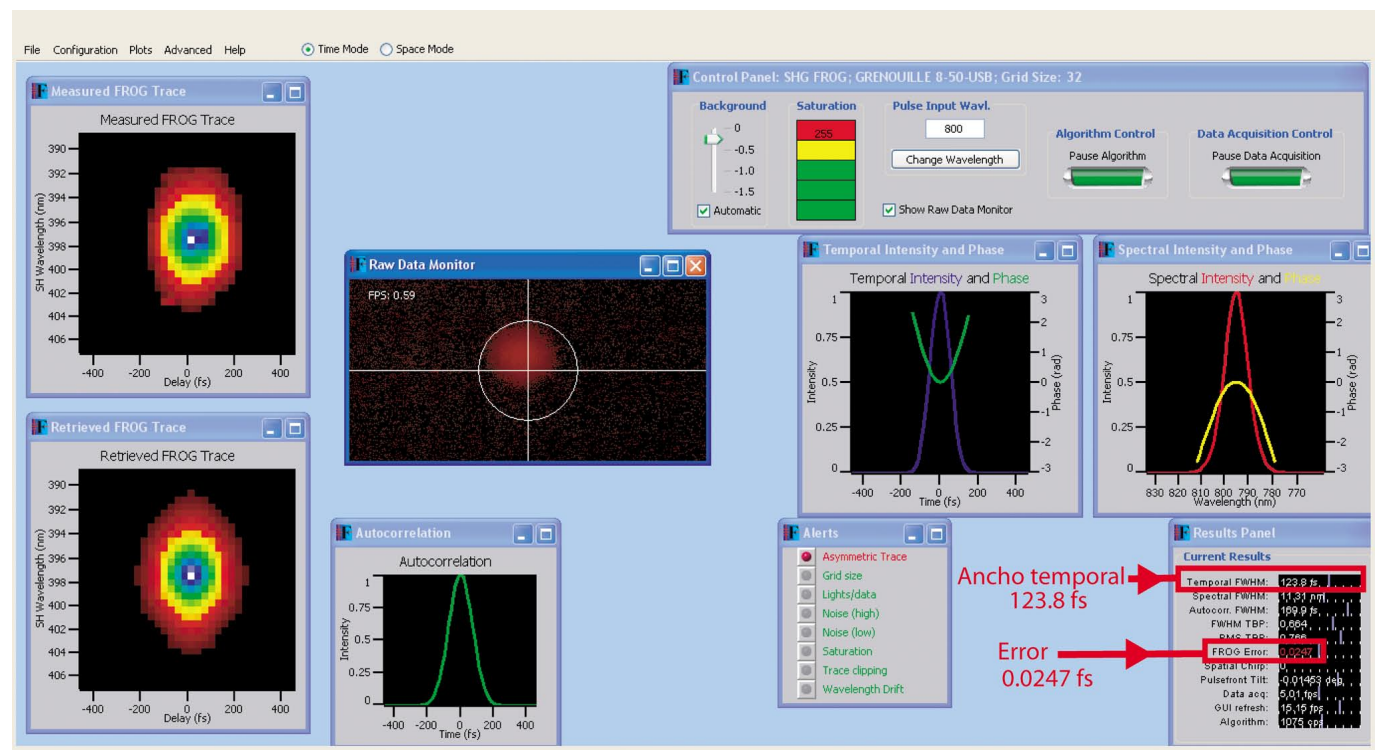

Figura 3.12: Medición del ancho del pulso temporal a través del uso del equipo comercial FROG.

polarizador. La importancia y consideraciones sobre cada uno de los elementos serán descriptos a lo largo de la presente sección.

En el caso de las antenas, para obtener un buen acoplamiento de la radiación hay que tener en cuenta lo siguiente [2] :

1. La radiación tiene mala direccionabilidad debido a que la onda es mas pequeña que el tamaño del haz. Esto es generado por el papel que juega la difracción en la propagación. También se puede decir que tal efecto es consecuencia de que el tamaño de la antena, es mucho menor que la longitud de onda.

2. Como las componentes frecuenciales generadas cubren más de una década en frecuencia, se generan diferencias en las características de la propagación para cada una de ellas.

3. Como el detector mide el campo eléctrico y no solo la intensidad del pulso de $\mathrm{THz}$, no es suficiente con solo medir la potencia. También es necesario que llegue todo el perfil espacial del campo al detector.

A continuación se realiza una breve descripción teórica sobre el problema comentado en el punto dos. Para describir dicho efecto se asume un patrón de radiación dipolar, donde la irradiancia luego de la lente en el campo lejano se 

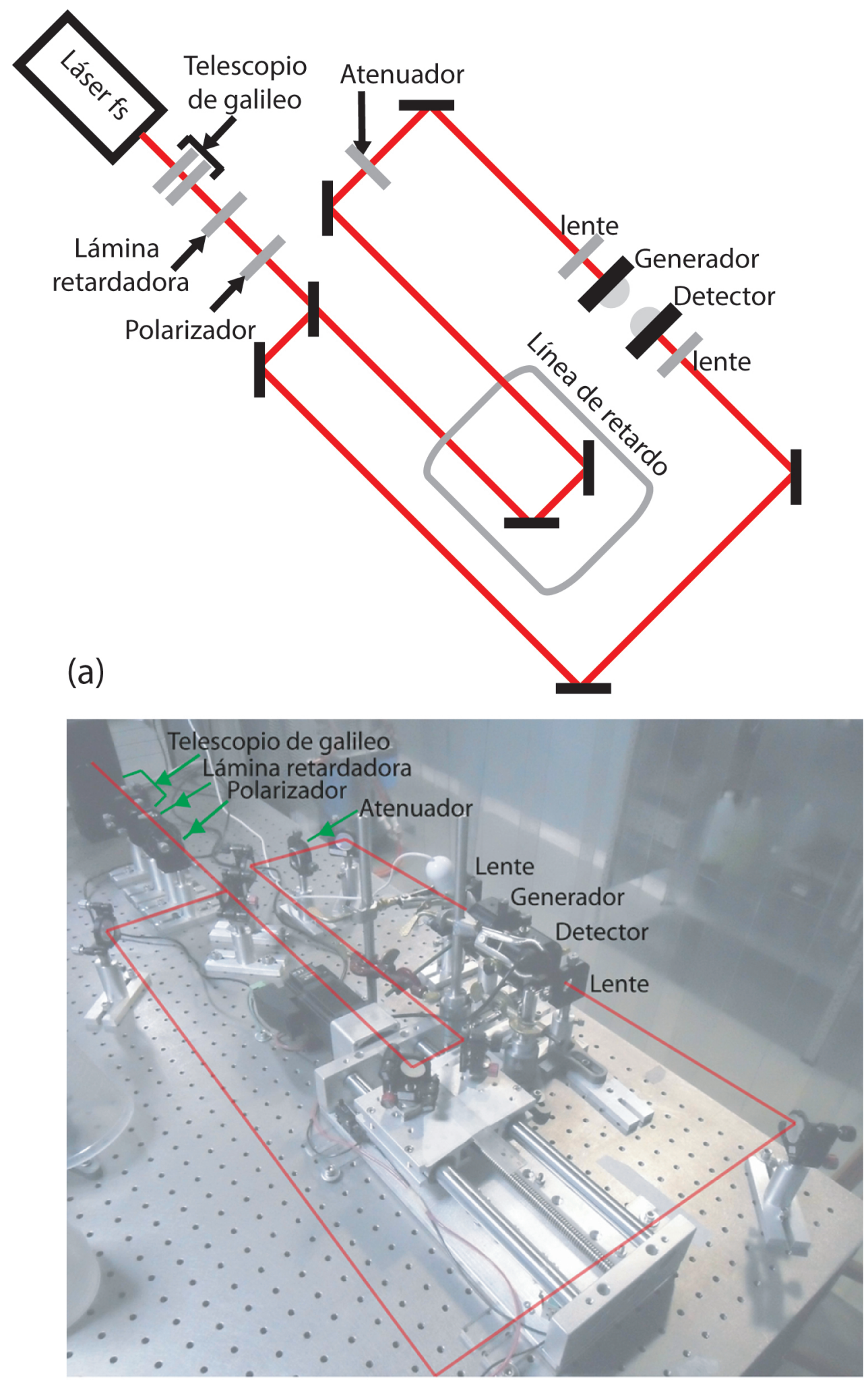

(b)

Figura 3.13: Configuración experimental del espectrómetro. (a) Esquema gráfico. (b) Foto del montaje experimental. 
comporta aproximadamente como una distribución Gaussiana. Entonces, suponiendo un haz Gaussiano con una cintura $w_{0}$, en la dirección de propagación $z=0$ y frecuencia $\omega$ el patrón del campo a una distancia $z$ esta dado por [2] :

$$
\begin{aligned}
E(\omega, r, z)= & E_{0}(\omega) \frac{w_{0}}{w(\omega, z)}\left[-\frac{r^{2}}{w^{2}(w, z)}\right] \exp \left[-j\left[\frac{z \omega}{c}-\tan ^{-1}\left(\frac{z}{Z_{0}(\omega)}\right)\right]\right] \\
& \exp \left[-j \frac{r^{2} \omega}{2 c R(\omega, z)}\right]
\end{aligned}
$$

donde $r$ es la distancia radial en el plano x-y y $Z_{0}$ es el intervalo de Rayleigh que se puede escribir como:

$$
Z_{0}(\omega)=\frac{\omega w_{0}^{2}}{2 c}
$$

La cintura del haz a una distancia $z$ en función de $\omega$, se puede escribir de la siguiente manera:

$$
w(\omega, z)=\sqrt{w_{0}^{2}\left[1+\frac{z 2 c}{\omega w_{0}^{2}}\right]} .
$$

En la Figura 3.14 se graficó la ecuación (3.18) para distintos valores de frecuencia. En la misma se evidencia claramente que para mayores frecuencias la cintura del haz es menor. Por tal motivo es necesario tener extremo cuidado en la dirección de propagación de la irradiación de la antena para poder detectar todas las frecuencias generadas.

Para solucionar algunos de los problemas anteriores se implementa la adición de una lente sobre el sustrato de la antena. La misma puede servir para colimar la radiación en el caso del generador (Fig. 3.15) [2, 54], o de enfocar en el caso del detector. Las antenas Teravil utilizadas en la tesis poseen una lente de silicio sobre el sustrato y tornillos móviles para direccionarla en el plano X-Y (Fig. 3.16).

A la hora de posicionar las antenas se tuvo en cuenta el retraso generado por las lentes de silicio al llegar la señal de THz al detector. Dado que las lentes tienen un índice de refracción de 3.5 y un espesor $d=1 \mathrm{~cm}$, se obtiene que el retraso es $\Delta t=2 d(n-1) / c$. Provocando espacialmente una diferencia entre las ramas del interferómetro de $5 \mathrm{~cm}$. Tal consideración en el posicionamiento de las antenas logró que la radiación de THz y el pulso de luz lleguen simultáneamente al detector. 


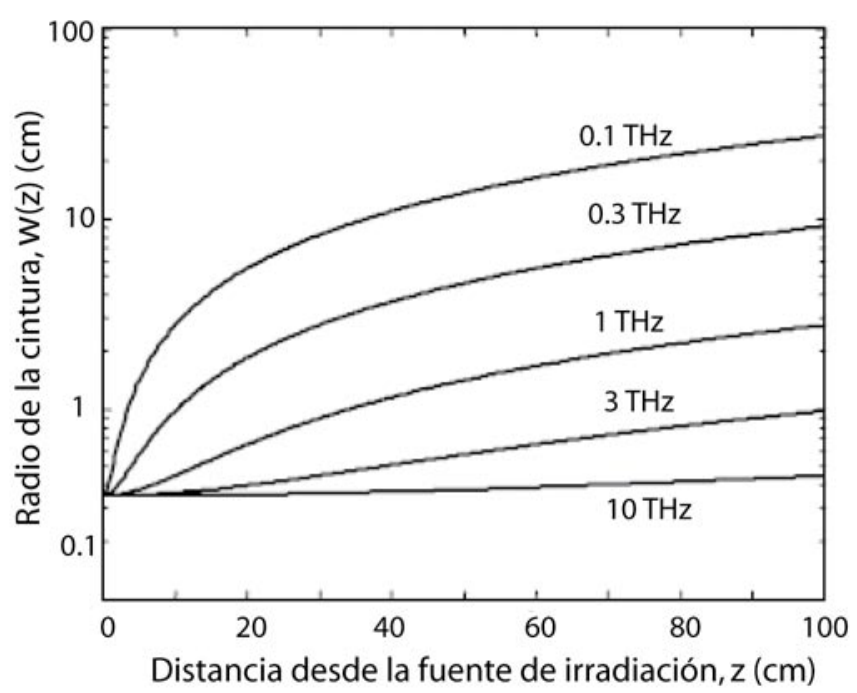

Figura 3.14: Gráfico de la ecuación (3.18) para distintos valores de frecuencia.

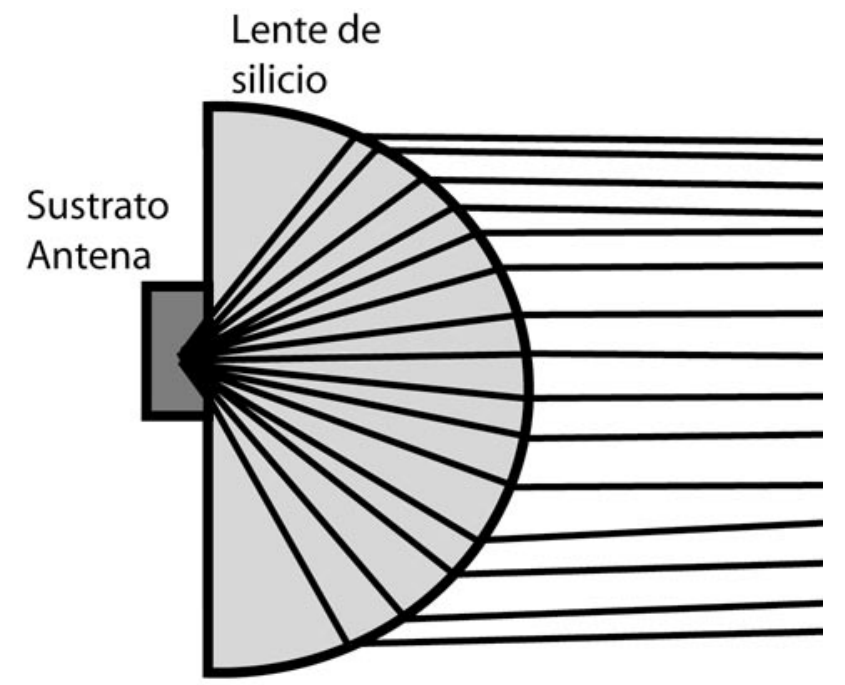

Figura 3.15: Lente colimadora de silicio con la realización de la traza de rayos. 

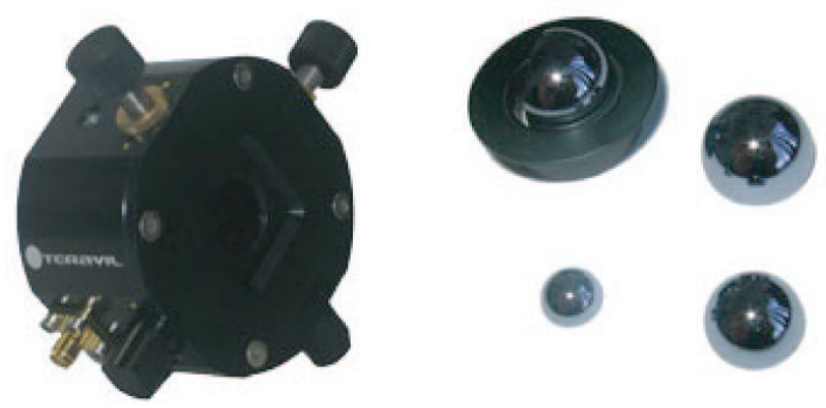

Figura 3.16: Lente de silicio Hyper-hemispherical de antena Teravils.

Para poder solucionar aún más el problema comentado en el punto dos, se utilizan otros elementos complementarios al uso de las lentes de silicio. Los más comunes son la implementación de lentes como se muestra en dos posibles configuraciones en la Fig. 3.17, o espejos parabólicos off-axis como se muestra en la Fig. 3.18. Aunque los últimos tienen la ventaja de no absorber la irradiación generada son muy complicados de alinear [21].

En el espectrómetro desarrollado, se acercaron lo más posible las antenas para asegurar que la totalidad de las frecuencias arribaran correctamente al detector. También, se variaron los tornillos de posicionamiento de las lentes de silicio hasta obtener la mayor intensidad de señal.

$\mathrm{Al}$ tener las muestras utilizadas un tamaño mayor al tamaño espacial de entrada de la antena detectora, se aseguraba que todas las frecuencias generadas en el espectrómetro hayan pasado a través de la misma.

\section{Potencia del láser y Enfoque}

El fabricante de las antenas fotoconductoras indica en las recomendaciones de uso, que las mismas sean irradiadas con una potencia media de $30 \mathrm{mw}$, no excediendo los $50 \mathrm{mw}$. Para lograr graduar la potencia del láser, se utilizó un polarizador y una lámina retardadora de media onda como se muestra en la Fig. 3.13 .

Con respecto al enfoque, los fabricantes sugieren que se irradie con un spot de $30 \mu \mathrm{m}$ entre los electrodos de las antenas. Por tal motivo, se calculó el mínimo spot teórico que era posible lograr con la instrumentación montada de la siguiente manera.

Al tener el láser un diámetro $d=1,2 \mathrm{~mm}$ incidiendo en una lente de distancia focal $f=5 \mathrm{~cm}$, el mínimo radio del spot logrado limitado por difracción esta dado por $R=1,22 \frac{\lambda f}{d}=41 \mu \mathrm{m}$. De esa manera, al estar por arriba del tamaño 

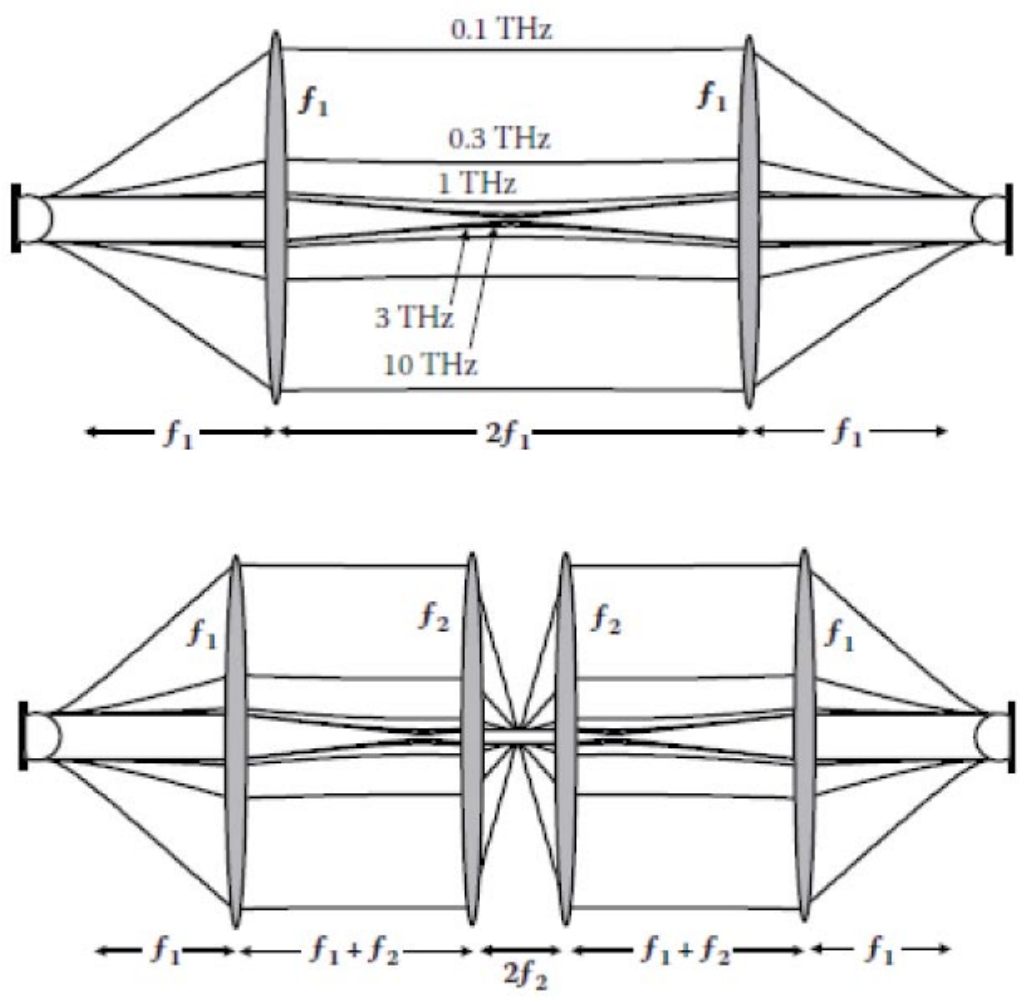

Figura 3.17: Configuraciones de lentes.

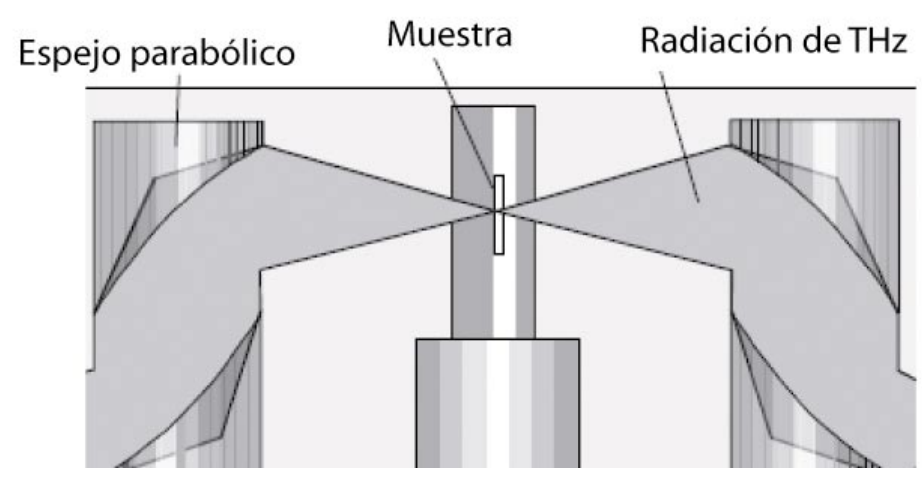

Figura 3.18: Configuración con espejos parabólicos. 


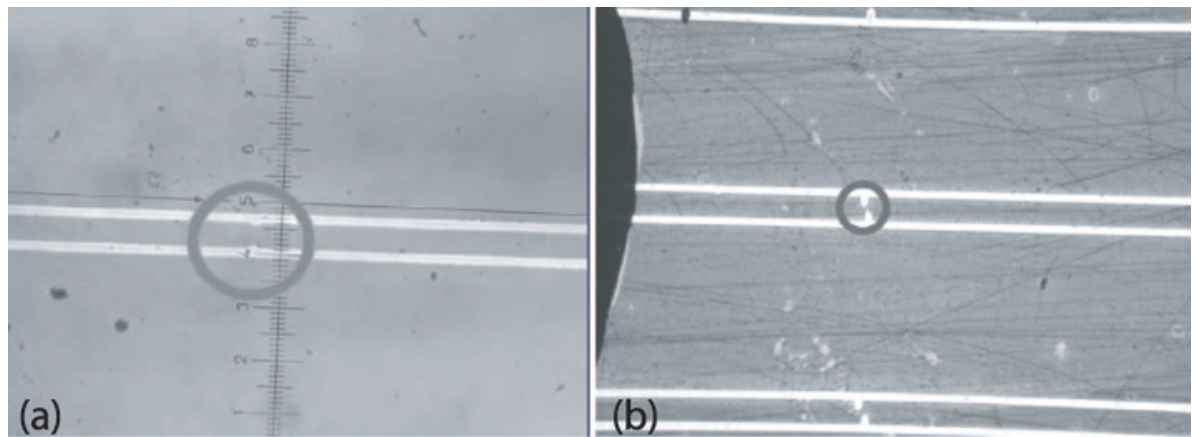

Figura 3.19: (a) Imagen del área de enfoque de la antena generadora. (b) Imagen del área de enfoque de la antena detectora.
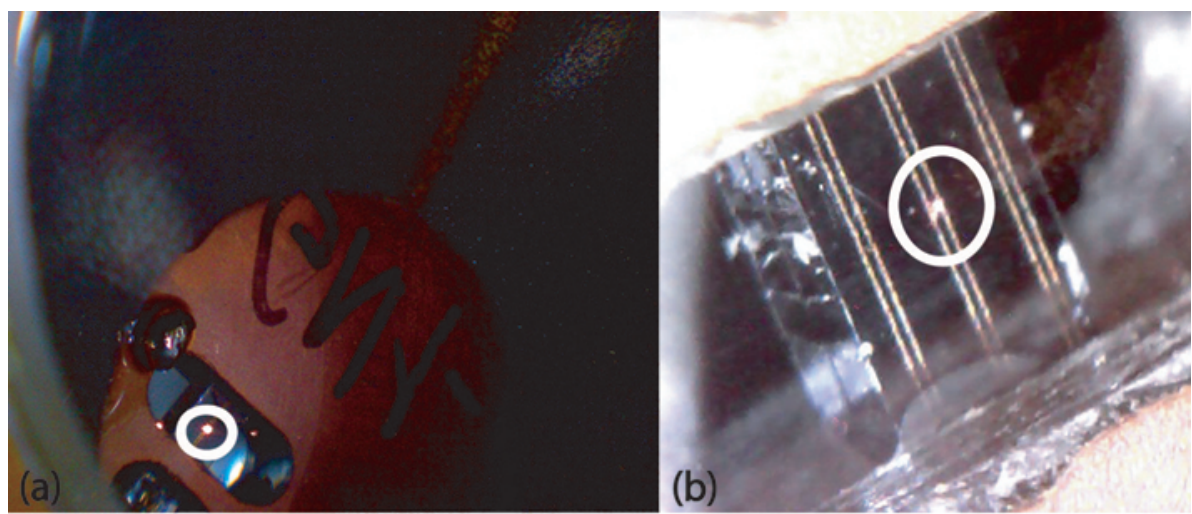

Figura 3.20: (a) Fotografía del enfoque logrado en la antena generadora. (b) Fotografía del enfoque logrado en la antena detectora. 
del spot sugerido, se implementó un telescopio tipo Galileo para aumentar el diámetro del haz (ver Fig. 3.13). El telescopio consistió en el uso de una lente divergente con focal $f=-25 \mathrm{~mm}$, y una lente convergente de focal $f=75 \mathrm{~mm}$ logrando amplificar X3 el haz láser. Dicho resultado que se obtuvo de hacer el cociente de ambas distancias focales posibilitó un spot de radio $R=13,6 \mu \mathrm{m}$.

En el caso del generador la irradiación tiene que ubicarse como se muestra en la Fig. 3.19(a), y en el detector como en la Fig. 3.19(b). Al tener que realizar un enfoque mas fino sobre el detector, la rama que contiene la línea de retardo se utilizó para irradiar el generador.

Para visualizar las áreas de enfoque sobre las antenas se utilizaron dos cámaras digitales. En la Figura 3.20 se muestran los resultados. Cabe mencionar que los spot se ven mas grandes de lo calculado teóricamente debido a la saturación de la cámara a la alta intensidad de los pulsos láser.

En las pruebas se observó que dependiendo a que electrodo del generador se acercaba el spot láser, variaba la emisión de THz. Esto ocurre, porque si el spot láser incide cerca del electrodo polarizado de manera positiva por la fuente de tensión, la fotocorriente generada en el material es menor que en el caso contrario [21]. Esto acontece, porque se genera transferencia de electrones en la juntura metal semiconductor, no contribuyendo activamente los pares electrón hueco generados en el proceso de radiación. Sin embargo, existe otro trabajo que obtiene un resultado contrario [55], por lo cual aún se encuentra dicha cuestión bajo discusión.

Por último, como los brazos del interferómetro no tenían la misma potencia en cada rama se adicionó un atenuador para solucionar el problema (ver Fig. $3.13)$.

\section{Dificultades y consideraciones}

Lo más complicado de lograr en el proceso de alineación fue el correcto enfoque sobre las antenas. Con las cámaras se podía ver la posición de los spots, pero no se podía tener certeza de que se estuviese trabajando en la distancia focal de las lentes. Por tal motivo se colocó la lente de enfoque del detector sobre un trasladador longitudinal, asegurando el correcto enfoque en el caso más crítico.

El procedimiento para lograr una señal satisfactoria en el detector, consistía en modificar sistemáticamente los tornillos de posicionamiento de las lentes de enfoque del trasladador y de las lentes de silicio hasta lograr obtener la mayor intensidad de señal.

Uno de los problemas en dicho procedimiento fue que la demora era de mucho tiempo para lograr obtener una señal satisfactoria. Esto se debía a que las 
monturas no quedaban perfectamente firmes, y porque los pasos de los tornillos de posicionamiento de las mismas no eran lo suficientemente pequeños. Esto también generaba que una vez montado el sistema, cualquier mínimo cambio provocaba grandes modificaciones en la detección de la señal generando inestabilidad en el sistema.

\subsection{Adquisición de datos y alimentación del generador de $\mathrm{THz}$}

A lo largo de la presente sección se detalla cada una de las partes del bloque dos de la Fig. 3.1.

\subsubsection{Amplificador de lock-in}

Se utilizó un amplificador de lock-in para medir la corriente del detector, ya que es adecuado para medir señales de baja intensidad como es el presente caso.

El amplificador de lock-in utilizado fue el modelo SR830 de la empresa Stanford research systems. En la Figura 3.21 se muestran sus principales características.

El funcionamiento del amplificador de lock-in se basa en obtener la señal mediante una detección sensible a la fase. Para poder realizar la medida se necesita una frecuencia de referencia con la que tendrá que ser excitado el experimento y con la cual deberá contar el amplificador de lock-in. En la Figura 3.22 se muestra una onda sinusoidal que es la señal medida de frecuencia de referencia $\omega_{r}$ con la que se excita el experimento. Obteniéndose como salida $V_{\text {sig }} \operatorname{sen}\left(\omega_{r} t+\theta_{\text {sig }}\right)$, con $V_{\text {sig }}$ la amplitud de la señal. Simultáneamente, como se muestra en la misma figura el amplificador de lock-in genera una referencia interna $V_{L} \operatorname{sen}\left(\omega_{L} t+\theta_{\text {ref }}\right)$. El SR830 primero amplifica la señal y luego la multiplica por la referencia del lock-in utilizando un detector sensible a la fase. En la salida del mismo se obtiene el producto de ambas señales:

$$
\begin{gathered}
V_{\text {det } 1}=V_{\text {sig }} V_{L} \operatorname{sen}\left(\omega_{r} t+\theta_{\text {sig }}\right) \operatorname{sen}\left(\omega_{L} t+\theta_{r e f}\right) . \\
V_{\text {det } 1}=\frac{1}{2} V_{\text {sig }} V_{L} \cos \left(\left(\omega_{r}-\omega_{L}\right) t+\theta_{\text {sig }}-\theta_{r e f}\right)-\frac{1}{2} V_{\text {sig }} V_{L} \cos \left(\left(\omega_{r}+\omega_{L}\right) t+\theta_{\text {sig }}+\theta_{\text {ref }}\right) .
\end{gathered}
$$

$\mathrm{Al}$ pasar dichas señales por un filtro pasabajo, solo permanece en la salida el primer término de la expresión anterior. Obteniendo así: 


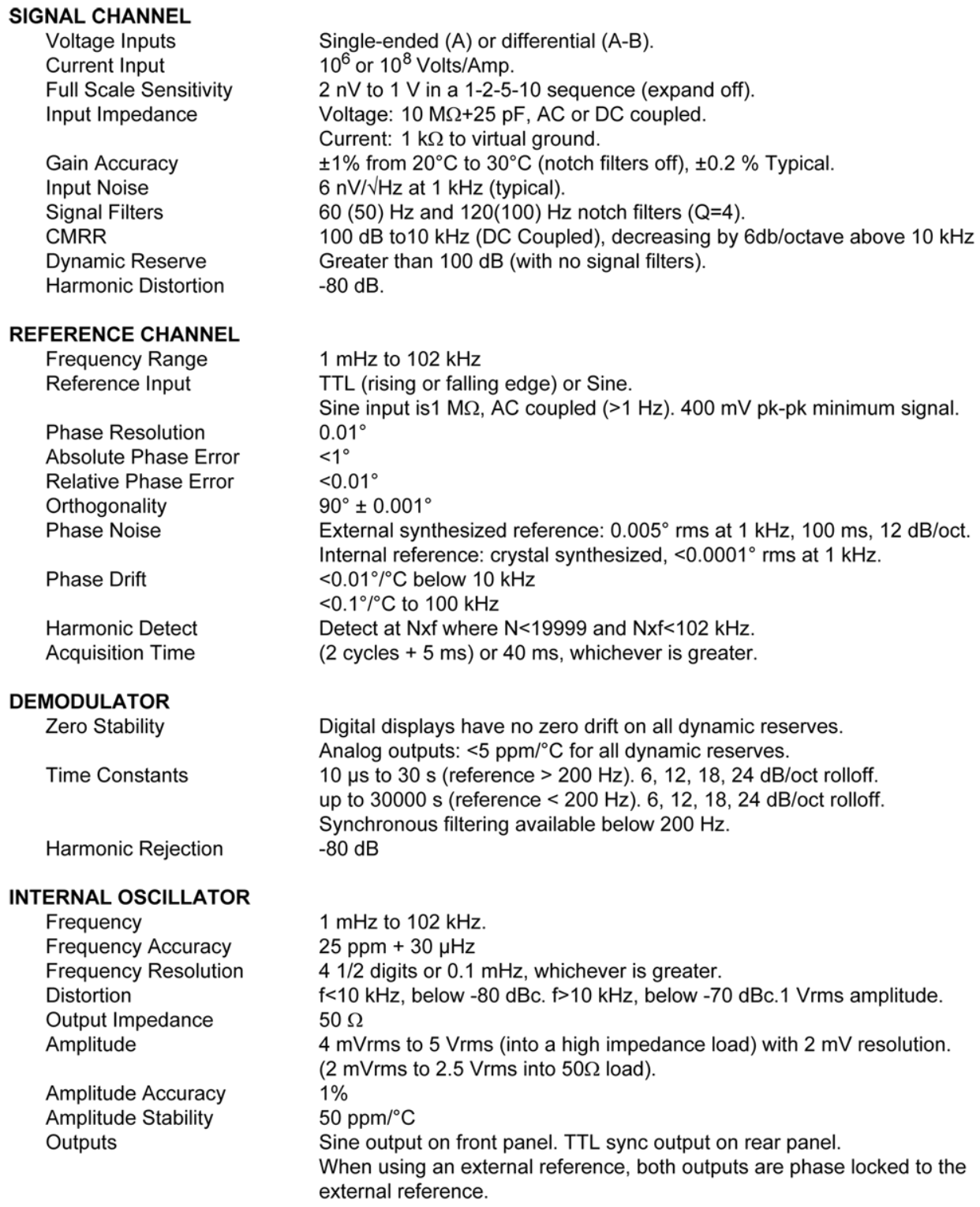

Figura 3.21: Características del amplificador de lock-in extraídas del manual del fabricante. 


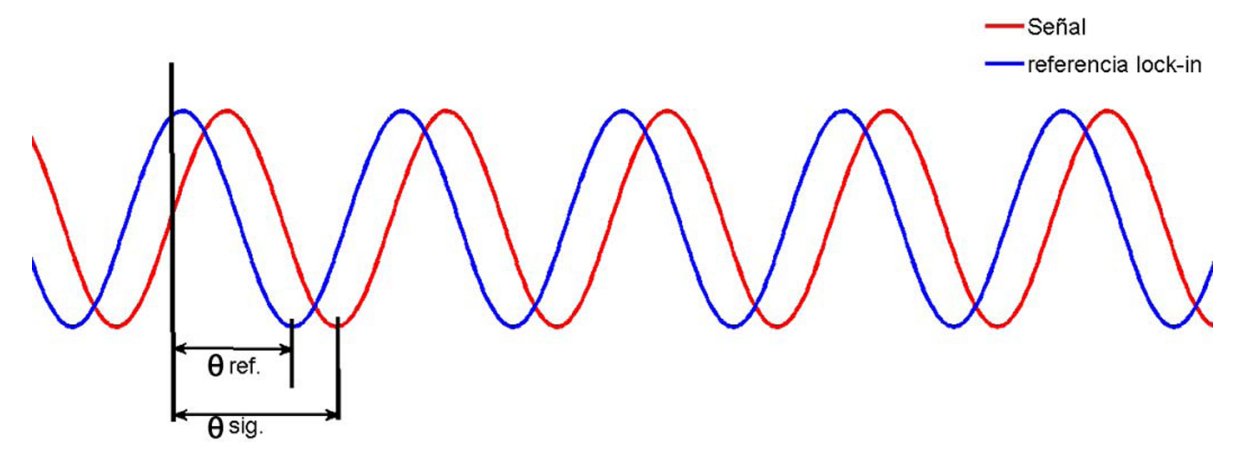

Figura 3.22: Señal del experimento modulada y señal de referencia del lock-in.

$$
V_{\text {det } 1}=\frac{1}{2} V_{\text {sig }} V_{L} \cos \left(\theta_{\text {sig }}-\theta_{\text {ref }}\right)=\frac{1}{2} V_{\text {sig }} V_{L} \cos (\theta) \sim V_{\text {sig }} \cos (\theta) .
$$

El único ruido que puede influir en el resultado es el que tenga la misma frecuencia que la referencia. Por tal motivo, se elige la frecuencia lo mas alta posible y que no sea un múltiplo de $50 \mathrm{~Hz}$.

Finalmente para obtener el módulo y la fase de la señal se utiliza otro multiplicador. Dicha operación se realiza entre la señal y la referencia desplazada $90^{\circ}$, generando como nueva referencia $V_{L} \operatorname{sen}\left(\omega_{L} t+\theta_{\text {ref }}+90\right)$. Luego de filtrar dicha multiplicación se obtiene:

$$
V_{\text {det } 2}=\frac{1}{2} V_{\text {sig }} V_{L} \operatorname{sen}\left(\theta_{\text {sig }}-\theta_{\text {ref }}\right) \sim V_{\text {sig }} \operatorname{sen}(\theta) .
$$

Llamando $X=V_{\text {sig }} \cos (\theta)$ e $Y=V_{\text {sig }} \operatorname{sen}(\theta)$, y haciendo las operaciones $R=\sqrt{X^{2}+Y^{2}}=V_{\text {sig }}$ y $\theta=\operatorname{atan}(Y / X)$, se obtiene el módulo y fase de la señal. Las operaciones son realizadas en el amplificador de lock-in que posibilita dichos resultados en sus salidas. Lo expuesto también es válido cuando la señal de entrada es una corriente, ya que el amplificador de lock-in cuenta con una entrada para dichas señales.

En nuestro caso particular para modular al sistema se utilizó primeramente un chopper mecánico (Modelo SR540, Stanford Research system). El mismo, obstruía el paso del láser generando la modulación de señal en el espectrómetro, y entregaba una señal cuadrada TTL con la frecuencia correspondiente al lockin.

De todas maneras, debido a que se buscaba modular con frecuencias mayores a las que proveía el chopper mecánico $(3.7 \mathrm{kHz})$ para minimizar la influencia del ruido se optó por otra opción. La misma, consistía en modular al sistema mediante la variación de la tensión de la antena generadora. Para lograrlo se empleó la referencia interna del lock-in que puede ser utilizada como salida. 
La cual proporciona una señal cuadrada o sinusoidal de hasta $102 \mathrm{kHz}$, con niveles de tensión TTL (0-5 V). Como la tensión (Vcc) de la antena tenía que estar en el rango de 15-50 V se decidió hacer una fuente de tensión regulada y un circuito adaptador TTL-Vcc (ver Fig. 3.1, bloque dos) que presente buena respuesta hasta $30 \mathrm{kHz}$.

\subsubsection{Tensión de alimentación de la antena y adaptador de tensión}

Tensión de alimentación

La tensión máxima de alimentación de las antenas fotoconductoras sugeridas por los fabricantes era de $50 \mathrm{~V}$, y la tensión aconsejada de operación $30 \mathrm{~V}$. Para cumplir dichas especificaciones se realizó una fuente con el uso del integrado LM317 logrando regular entre 15-50 V con una corriente máxima de 1.5 A.

En la Figura 3.23 se muestra el esquemático, el diagrama del PCB, y la foto del circuito desarrollado.

Adaptador de tensión (TTL-Vcc)

Para alimentar la antena generadora con la referencia interna del lock-in, se realizó un circuito para adaptar la señal de referencia de niveles TTL del lock-in a la tensión Vcc de la fuente. Para su realización se utilizó una compuerta lógica inversora modelo ULN2001A, que presentó una buena respuesta hasta $35 \mathrm{kHz}$ de modulación. El esquemático, el PCB y la foto del mismo se muestra en la Fig. 3.24.

\section{Consideraciones sobre el conexionado}

$\mathrm{Al}$ conectar todos los elementos se tuvo especial cuidado en el conexionado de las tierras del sistema. En particular se busco conectar a tierra o de manera flotante, las antenas, la mesa óptica, el lock-in, la fuente Vcc, el motor, la PC y el adaptador de tensión, de manera que no se generara un bucle de circulación de corriente en ninguna parte del sistema. Ya que no tener tal consideración afectaría las mediciones y podría poner en riesgo a los equipos y al usuario.

\subsubsection{Software de adquisición}

A medida que se realizaban los barridos temporales de las mediciones, se descargaban los datos obtenidos del lock-in a la PC mediante un software desarrollado en Matlab. Mediante el mismo se podía configurar el amplificador de 

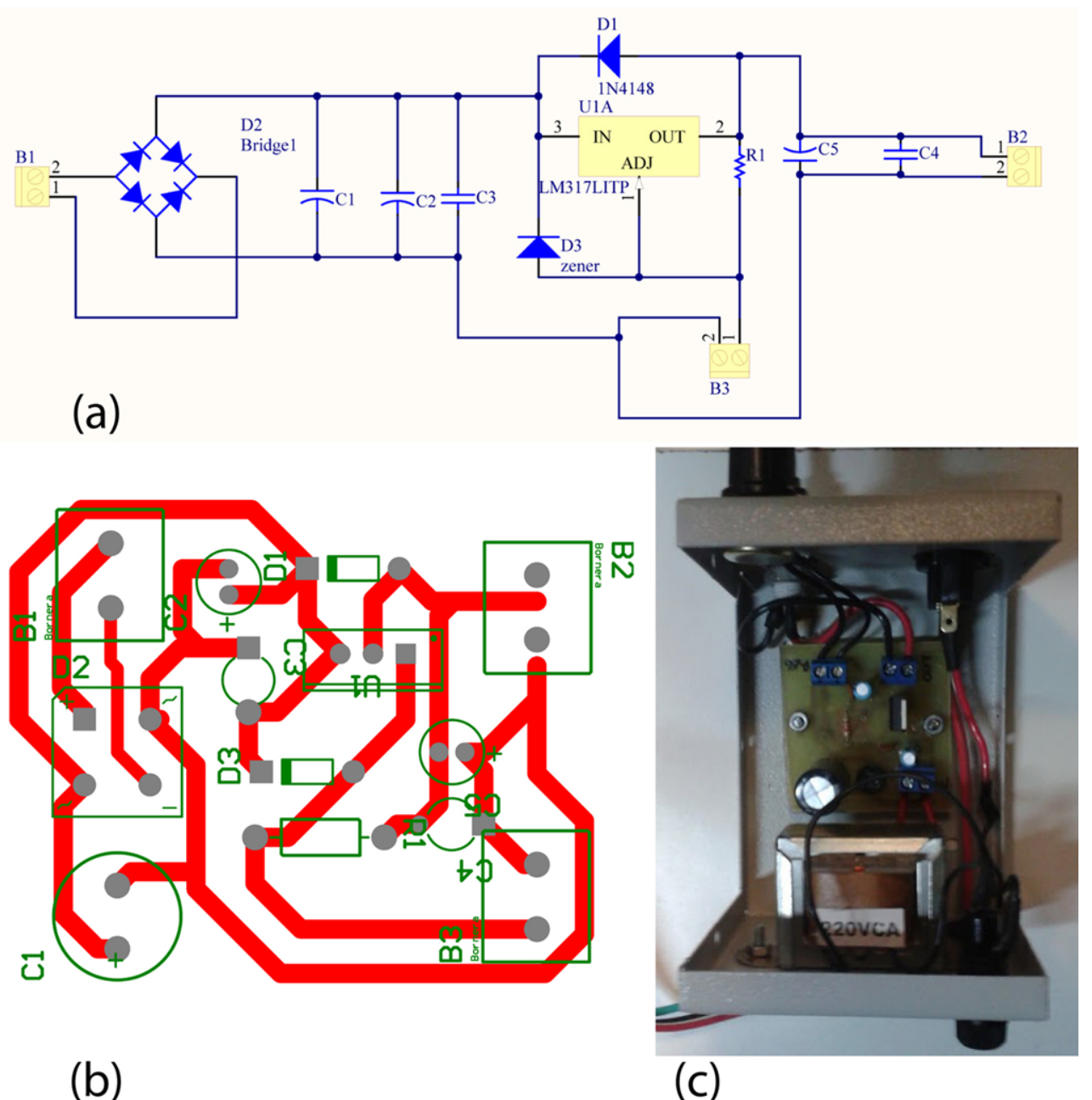

Figura 3.23: (a) Esquemático de la fuente de tensión. (b) PCB. (c) Foto del circuito realizado. 

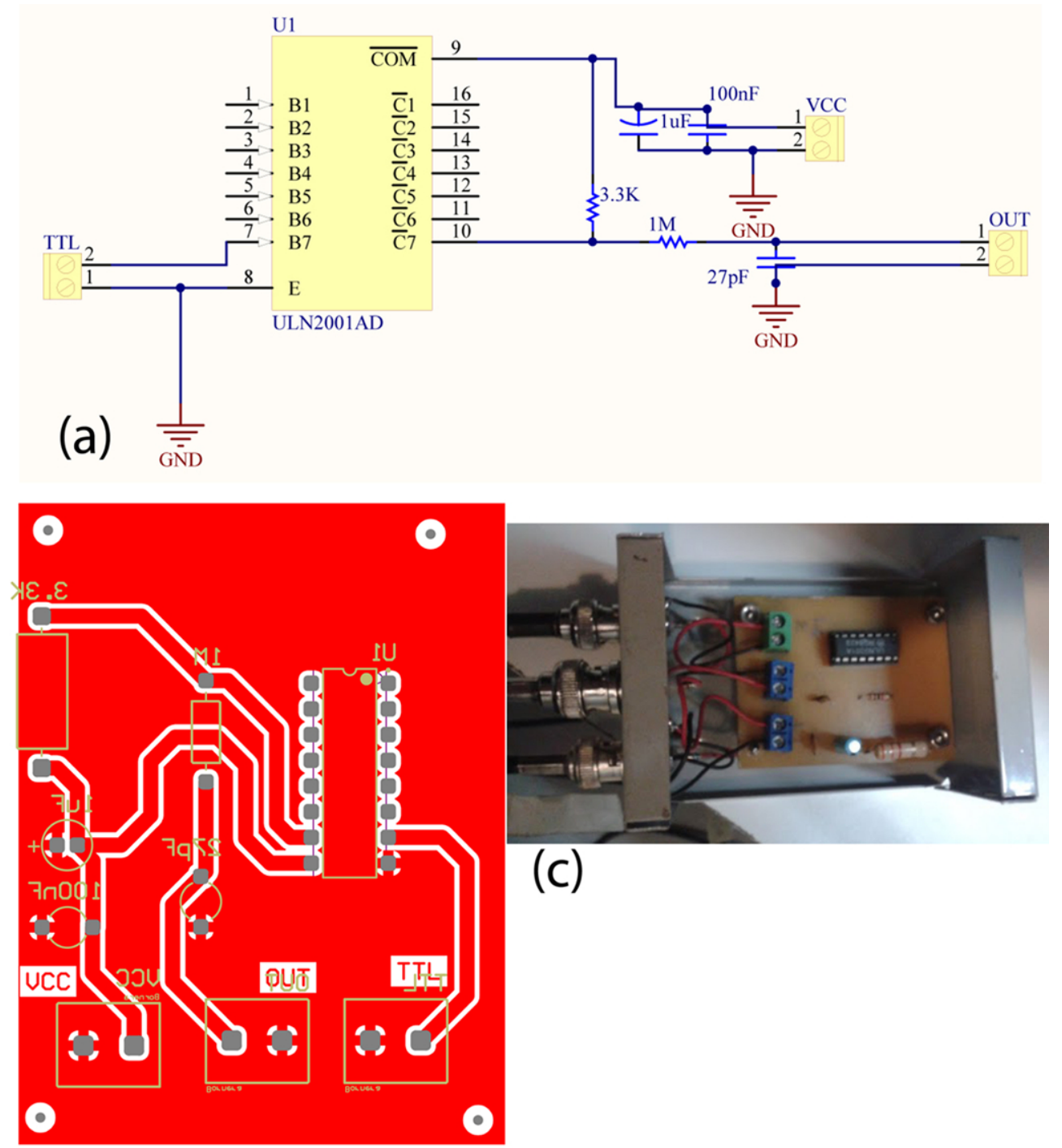

(b)

Figura 3.24: (a) Esquemático del adaptador de tensión. (b) PCB. (c) Foto del circuito realizado. 
lock-in, y se podía descargar los datos en un archivo .txt para su posterior procesamiento. También se presentaba en pantalla una figura con el mapeo del campo eléctrico mientras se hacia el barrido. En el Anexo I del Apéndice A, se presenta el programa desarrollado.

\subsection{Resultados espectrales del vapor de agua}

Con todos los elementos necesarios en la mesa óptica y con la alineación adecuada, se procedió a detectar el pulso de THz. Lo primero que se realizó para verificar el buen funcionamiento del sistema fue tomar mediciones del vapor de agua ambiente para compararlas con las líneas espectrales publicadas [56, 57]. Para llevarlo a cabo, se realizó un barrido temporal de 230 ps con una tensión de alimentación de $40 \mathrm{~V}$, una frecuencia de modulación de la tensión de la antena de $30 \mathrm{kHz}$, y con una potencia media del láser de $30 \mathrm{mw}$ en cada antena.

$\mathrm{Al}$ amplificador de lock-in se lo configuró de la siguiente manera:

-Comunicación RS232.

-Referencia interna con frecuencia de $30 \mathrm{KHz}$.

-Entrada de corriente (1 Mohm).

-DC coupling.

-2 filtros -Nocht de línea.

$-1 \mathrm{nA}$ de sensisibilidad.

-Constante de tiempo $30 \mathrm{~ms}$.

-Filtro $6 \mathrm{~dB} /$ oct.

Finalmente, para adquirir los datos se puso en movimiento la línea de retardo para la realización del mapeo del campo eléctrico.

Los resultados obtenidos se muestran en la Fig. 3.25. En la misma se presenta el pulso temporal junto al espectro obtenido de hacerle la transformada discreta de Fourier. En la traza temporal se evidencia una buena relación señalruido $(<22 \mathrm{~dB})$, con un ancho temporal del pulso de alrededor del picosegundo, como es usual en este tipo de sistemas. Con respecto al espectro, se observa una satisfactoria señal hasta al menos $1.8 \mathrm{THz}$. Eso es debido a que se encontró correspondencia entre las líneas de absorción del vapor de agua publicadas [57] y señaladas en la Fig. 3.25 hasta 1.72 THz. A continuación se muestra una tabla comparando dichos valores: 


\begin{tabular}{|l|l|}
\hline $\begin{array}{l}\text { Líneas de absorción } \\
\text { publicadas }[57](\mathrm{THz})\end{array}$ & $\begin{array}{l}\text { Líneas de absorción } \\
\text { medidas }(\mathrm{THz})\end{array}$ \\
\hline 0.557 & 0.556 \\
\hline 0.753 & 0.750 \\
\hline 0.989 & 0.987 \\
\hline 1.098 & 1.100 \\
\hline 1.164 & 1.164 \\
\hline 1.411 & 1.414 \\
\hline 1.603 & 1.604 \\
\hline 1.671 & 1.669 \\
\hline 1.718 & 1.716 \\
\hline
\end{tabular}

Tabla 3.1: Líneas espectrales del agua.

Las diferencias en los resultados se deben al uso de distintas resoluciones espectrales, y a la diferencia en la relación señal-ruido de los sistemas. En nuestro caso particular la resolución espectral fue de $1 / 230 \mathrm{ps}=4,35 \mathrm{GHz}$.

Con los primeros resultados obtenidos se publicó un trabajo en la revista nacional Anales AFA [58] de la Asociación Física Argentina. 

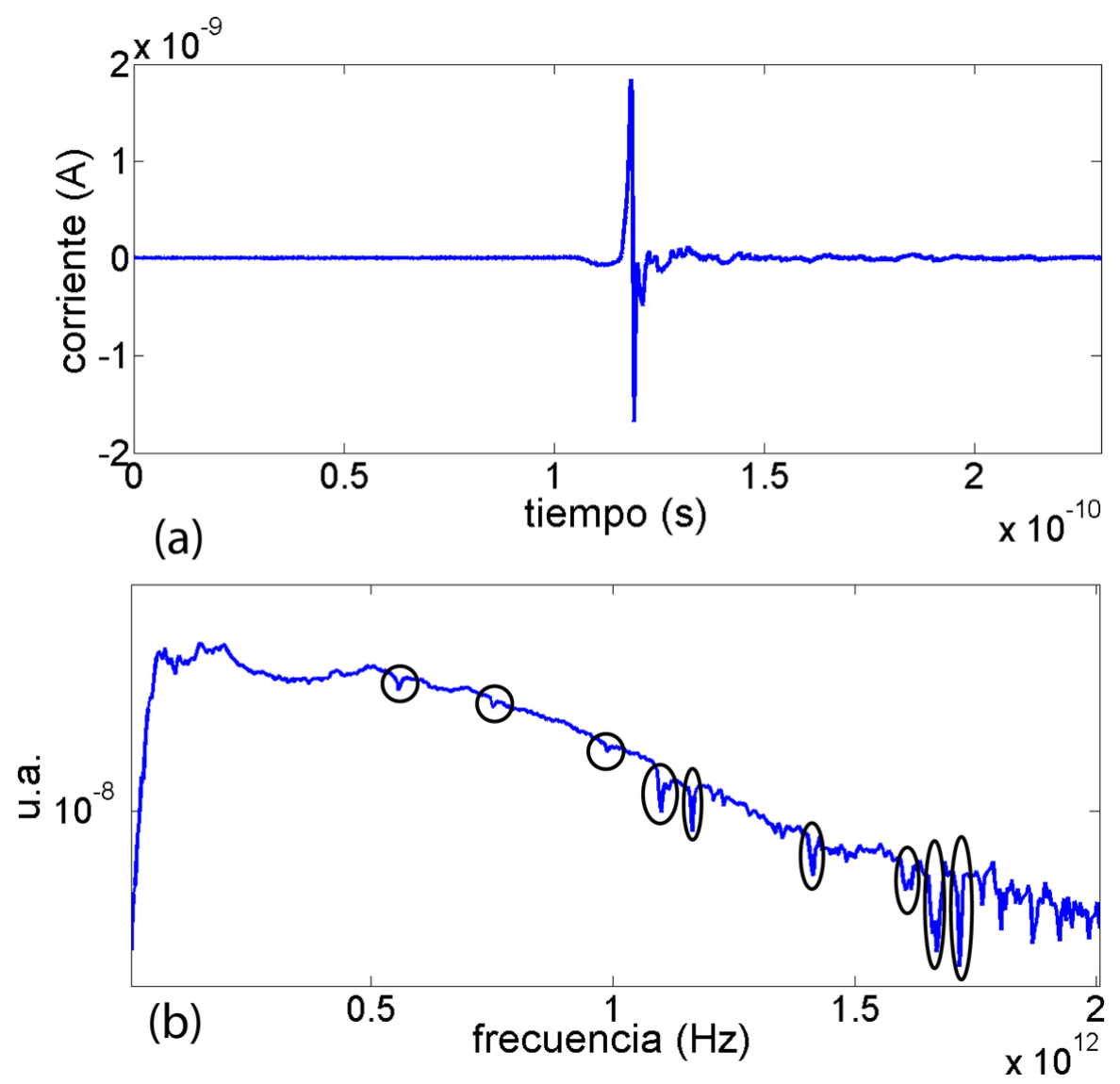

Figura 3.25: (a) Traza temporal del vapor de agua. (b) Espectro del vapor de agua. 


\section{Capítulo 4}

\section{Teoría del procesamiento de datos. Resultados espectrales y temporales de materiales}

\subsection{Análisis frecuencial}

Para obtener los espectros por absorción de los materiales se necesita convencionalmente realizar el mapeo del campo eléctrico dos veces ( 2 barridos). Uno sin muestra presente al que se llama referencia, y otro con la muestra. A ambas mediciones temporales se les realiza la transformada discreta de Fourier para obtener las componentes frecuenciales de las señales.

Utilizando (2.4), la corriente de referencia en el dominio frecuencial se puede escribir como $I_{r e f}(\omega) \propto Q(\omega) P(\omega) E_{T H z-r e f}(\omega)$, donde $Q(\omega)$ es el espectro de la excitación láser, y $P(\omega)$ es la respuesta en frecuencia de los fotoportadores en el semiconductor. De igual manera podemos escribir en el caso en que se encuentre la muestra presente $I_{\text {muestra }}(\omega) \propto Q(\omega) P(\omega) E_{\text {THz-muestra }}(\omega)$.

Considerando la Fig. 4.1, donde no se tiene en cuenta ninguna reflexión por trasmisión en el material, se obtiene [30]:

$$
E_{\mathrm{THz}-\text { muestra }}(\omega)=E_{0}(\omega) t_{12} t_{23} e^{-\alpha \mathrm{d} / 2} e^{j n \omega \mathrm{d} / \mathrm{c}}
$$

donde $t_{12}$ y $t_{23}$ son los coeficientes de trasmisión de Fresnel, $e^{j n \omega \mathrm{d} / \mathrm{c}}$ es el coeficiente de propagación, y $e^{-\alpha \mathrm{d} / 2}$ es la atenuación en el material.

Cuando no hay muestra presente, el campo eléctrico recibido se puede escribir como:

$$
E_{\mathrm{THz}-\mathrm{ref}}(\omega)=E_{0}(\omega) e^{j \omega \mathrm{d} / \mathrm{c}}
$$




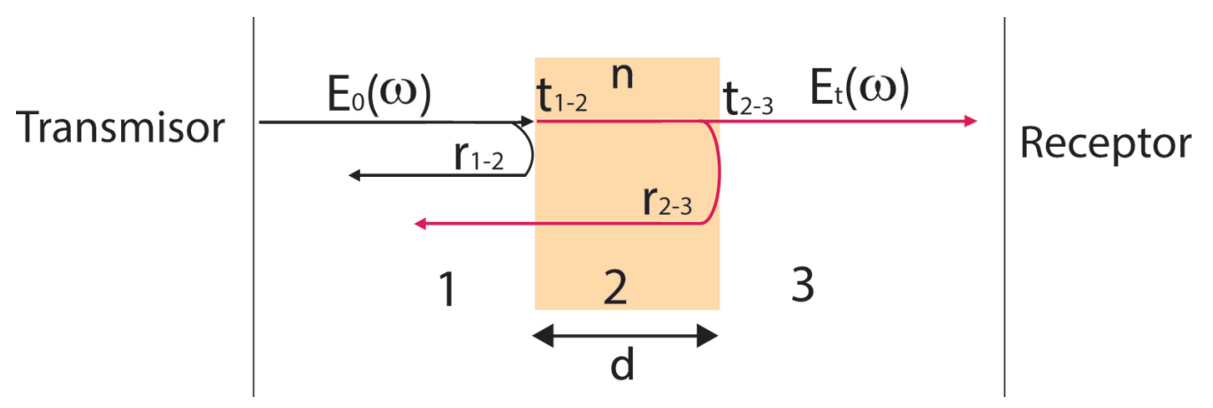

Figura 4.1: Esquema de la geometría de espectroscopía por transmisión.

Finalmente, escribiendo la función transferencia con la relación entre la corriente de referencia y con muestra, se obtiene:

$$
\begin{gathered}
T(\omega)=|T(\omega)| e^{j \phi(\omega)}=\frac{I_{\text {muestra }}(\omega)}{I_{\text {ref }}(\omega)}=\frac{Q(\omega) P(\omega) E_{\mathrm{THz}-\text { muestra }}(\omega)}{Q(\omega) P(\omega) E_{\mathrm{THz}-\mathrm{ref}}(\omega)}= \\
=\frac{E_{0}(\omega) t_{12} t_{23} e^{-\alpha \mathrm{d} / 2} e^{j n \omega \mathrm{d} / \mathrm{c}}}{E_{0}(\omega) e^{j \omega \mathrm{d} / \mathrm{c}}}=t_{12} t_{23} e^{-\alpha \mathrm{d} / 2} e^{j(n-1) \omega \mathrm{d} / \mathrm{c}} .
\end{gathered}
$$

Si limitamos el análisis a muestras con baja absorción, los coeficientes de Fresnel de transmisión son reales, y considerando incidencia normal del campo eléctrico se obtiene:

$$
\begin{gathered}
n(\omega)=1+\frac{\phi(\omega) c}{\omega \mathrm{d}} . \\
\alpha(\omega)=-\frac{2}{\mathrm{~d}} \ln \left(\frac{(n+1)^{2}}{4 n}|T(\omega)|\right) .
\end{gathered}
$$

En general, la mayoría de las muestras utilizadas cumplen la condición de baja absorción (i.e. plásticos, madera, telas, etc.), siendo válidas (4.4) y (4.5). En los casos en que haya una absorción apreciable se generan cambios en la fase de los parámetros ópticos, debido a que los coeficientes de Fresnel tienen una forma compleja. Esto ocurre en muestras delgadas de alta absorción como es el caso del agua o de semiconductores altamente dopados, en los cuales los cambios de fase debido a los coeficientes de Fresnel es comparable al cambio de fase debido a la propagación de la onda electromagnética por la muestra. Un correcto análisis de dichos casos se puede encontrar en la referencia [30].

A continuación, se realiza el desarrollo teórico en el caso de considerar las reflexiones por trasmisión en el material (Fig. 4.2). En la Fig. 4.3(a) se pueden ver los ecos producto de tal efecto. El análisis se basa en lo publicado en el 


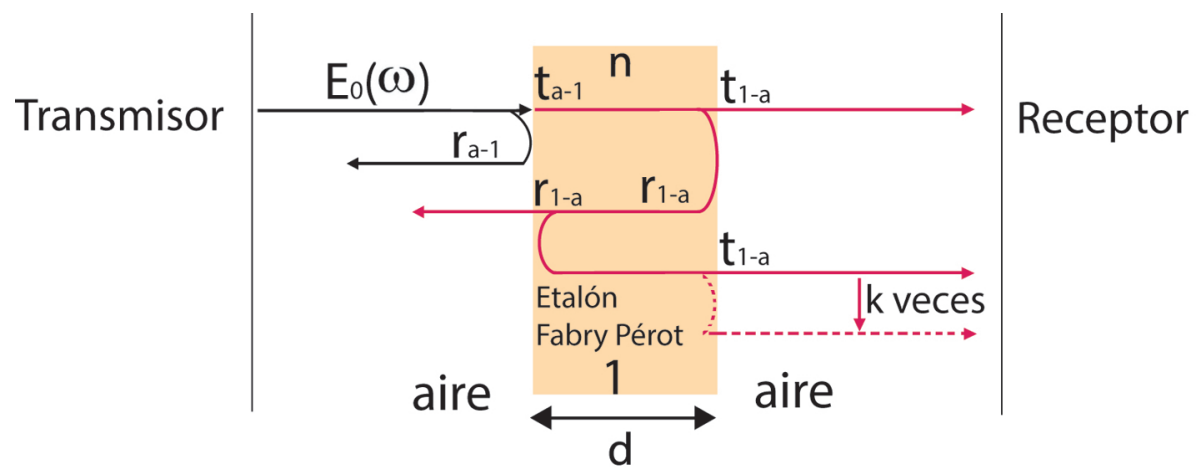

Figura 4.2: Esquema de la geometría de espectroscopía por transmisión.

trabajo científico de la referencia [59]. EL mismo considera una muestra de longitud d en aire y se asume lo siguiente:

-La muestra no tiene rugosidad apreciable y tiene las caras paralelas.

-La respuesta electromagnética en la muestra es lineal.

-También se supone la onda con incidencia normal, polarización lineal y paralela al eje óptico de la muestra (en el caso en que presente anisotropía óptica).

Con tales consideraciones el campo eléctrico a la salida del material se puede escribir como:

$$
\begin{aligned}
E_{\text {muestra }}(\omega)= & E_{0}(\omega) t_{1 a} t_{a 1} P_{1} e^{-\alpha \mathrm{d} / 2} \sum_{k=0}^{\infty}\left(r_{1 a}^{2} P_{1}^{2}\right)^{k} \\
& =E_{0}(\omega) \frac{4}{\left(n_{1}+1\right)^{2}} e^{j n w \mathrm{~d} / \mathrm{c}} e^{-\alpha d / 2} \sum_{k=0}^{\infty}\left(\frac{\left(n_{1}-1\right)^{2}}{\left(n_{1}+1\right)^{2}} e^{j 2 n w \mathrm{~d} / \mathrm{c}} e^{-\alpha d / 2}\right)^{k} .
\end{aligned}
$$

El campo eléctrico de referencia se puede escribir como en (4.2), obteniendo la función transferencia siguiente:

$$
\begin{aligned}
T(\omega)= & \frac{E_{\text {muestra }}}{E_{\text {ref }}}=t_{1 a} t_{a 1} P_{1} e^{-\alpha \mathrm{d} / 2} \sum_{k=0}^{\infty}\left(r_{1 a}^{2} P_{1}^{2}\right)^{k} \\
& =\frac{4}{\left(n_{1}+1\right)^{2}} e^{j(n-1) w \mathrm{~d} / \mathrm{c}} e^{-\alpha \mathrm{d} / 2} \sum_{k=0}^{\infty}\left(\frac{\left(n_{1}-1\right)^{2}}{\left(n_{1}+1\right)^{2}} e^{j 2 n w \mathrm{~d} / \mathrm{c}} e^{-\alpha \mathrm{d}}\right)^{k} .
\end{aligned}
$$

Considerando la sumatoria como una serie geométrica, se obtiene:

$$
T(\omega)=\frac{\frac{4}{\left(n_{1}+1\right)^{2}} e^{j(n-1) w \mathrm{~d} / \mathrm{c}} e^{-\alpha \mathrm{d} / 2}}{1-\left(\frac{\left(n_{1}-1\right)^{2}}{\left(n_{1}+1\right)^{2}} e^{j 2 n w \mathrm{~d} / \mathrm{c}} e^{-\alpha \mathrm{d}}\right)}=\frac{4}{\left(n_{1}+1\right)^{2}} e^{j(n-1) w \mathrm{~d} / \mathrm{c}} e^{-\alpha \mathrm{d} / 2} F P(\omega),
$$


donde $F P(\omega)$ es el factor Fabry-Perot que considera los ecos de la señal. De no considerarse las reflexiones, el término $F P$ es uno, obteniéndose el mismo resultado presentado en la ecuación (4.3).

Los ecos de la señal temporal generan en el espectro una modulación en frecuencia no deseable (Fig. 4.3(b)), con lo cual es deseable eliminar las reflexiones. La manera mas simple de eliminar su influencia es a través del truncamiento temporal de la señal. Otra manera, es a través de un procesamiento de señal mas sofisticado como el propuesto en [60].

Aunque hay casos, en que es deseable trabajar solo con los ecos ya que presentan una varianza menor al pulso principal [61]. También son útiles cuando se desea obtener tanto los parámetros ópticos como el espesor del material [62]. Ya que mediante el procedimiento convencional se necesita saber el espesor de la muestra a priori.

\subsubsection{Resultados}

En la Figura 4.4 se muestran los resultados de los índices de refracción del zafiro y sílica utilizando la ecuación (4.4). Las señales se truncaron temporalmente de manera de eliminar los ecos. El espectrómetro se configuró con los mismos parámetros descriptos en la Sección 3.3. Los resultados obtenidos están deacuerdo con lo publicado en [63].

\subsection{Análisis temporal}

Otra manera de calcular el índice de refracción y la absorbancia de los materiales es a través del uso de los datos temporales. Se utiliza la información del retardo provocado en el pulso temporal cuando hay muestra presente, y cuando no la hay. El desarrollo teórico descripto a continuación fue publicado en el Latin America Optics and Photonics Conference (LAOP) [64].

A través del retardo generado entre los pulsos se calcula el índice de refracción :

$$
n=1+\mathrm{ct}_{d} / \mathrm{d}
$$

donde $t_{d}$ es el retardo entre los picos de los pulsos y d es el ancho de la muestra. Esto es válido suponiendo el índice de refracción constante en el ancho de banda del experimento.

También, la absorbancia se calcula de la siguiente manera:

$$
\alpha=\frac{2}{\mathrm{~d}} \ln \frac{4 n E_{r 0}}{(1+n)^{2} E_{s 0}}
$$



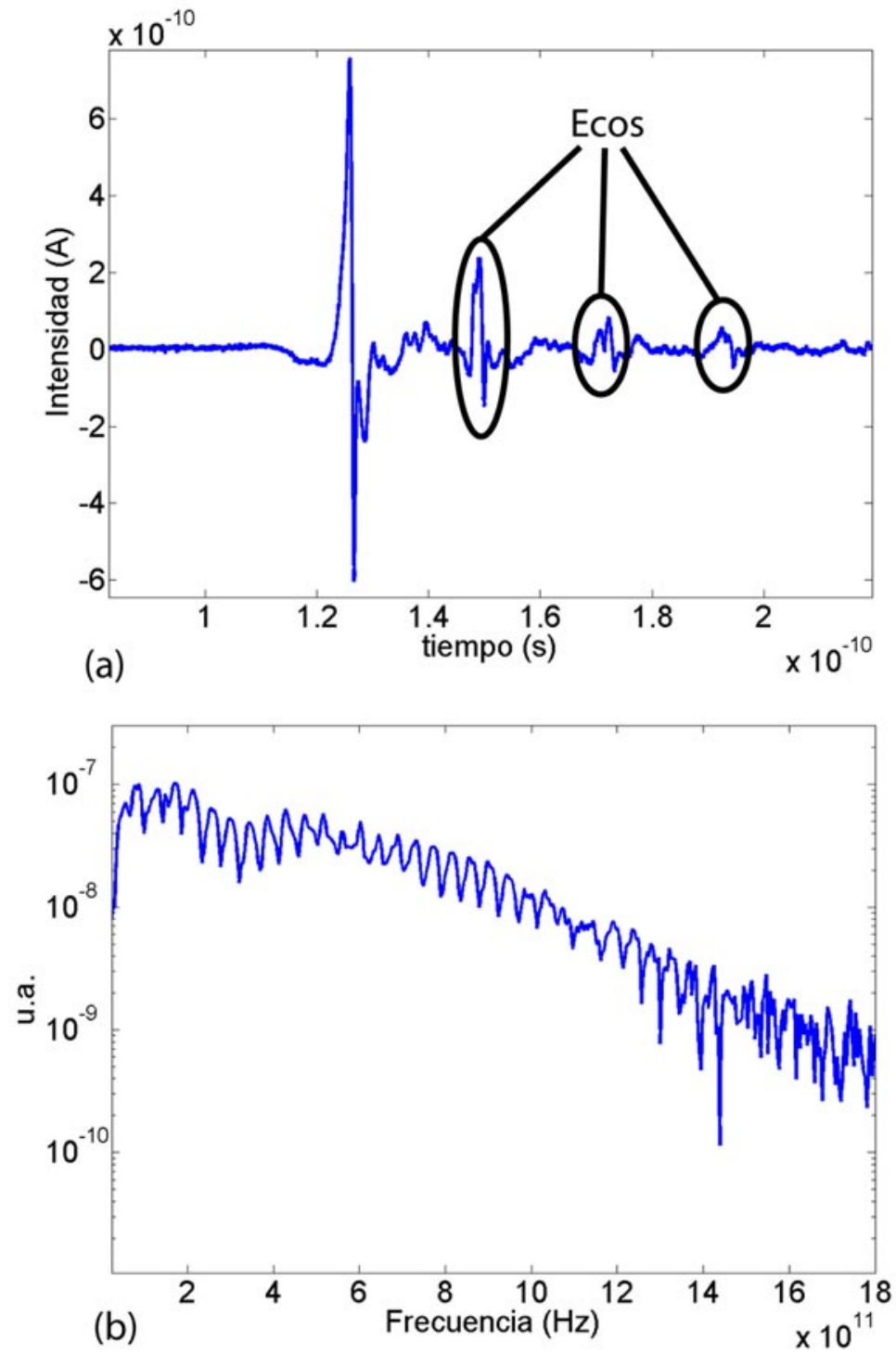

Figura 4.3: (a) Señal temporal de una muestra de zafiro. (b) Espectro de la traza temporal de (a). 

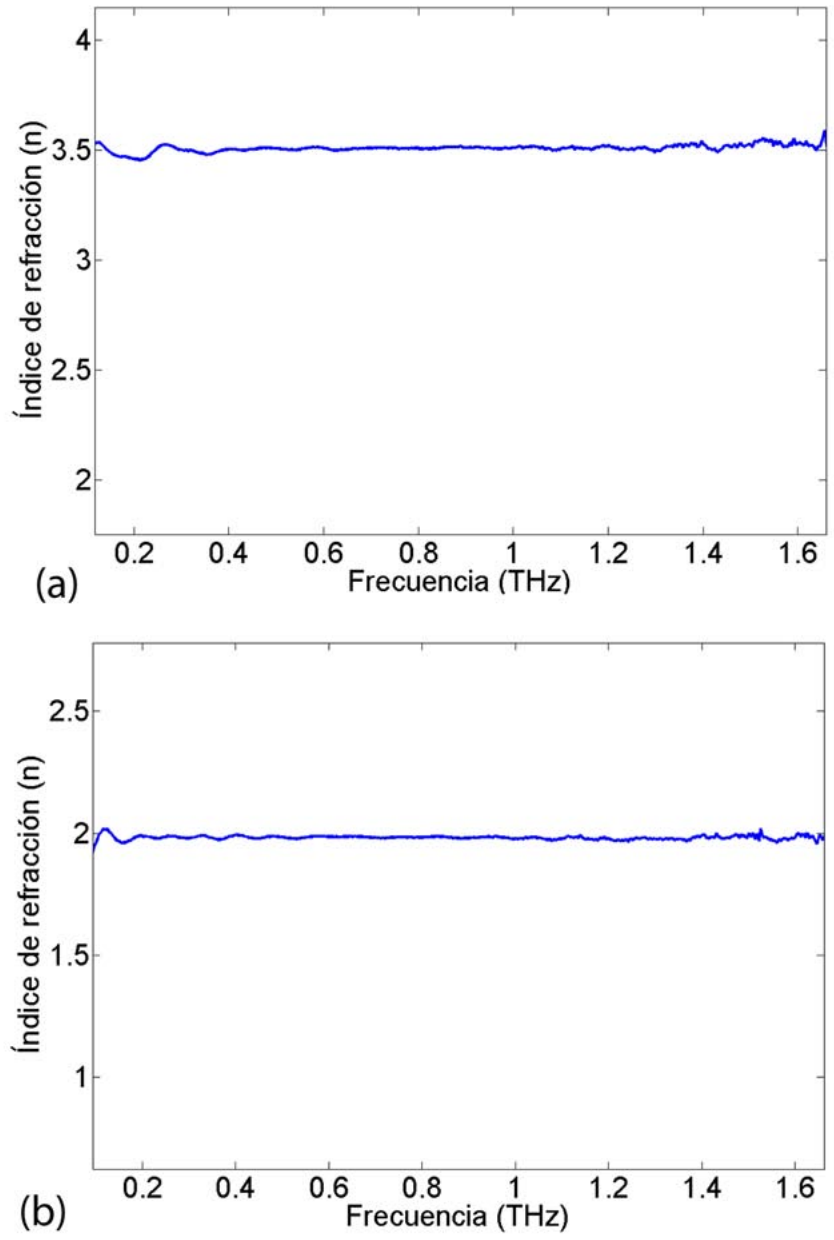

Figura 4.4: (a) Índice de refracción de una muestra de zafiro. (b) Índice de refracción de una muestra de sílica. 

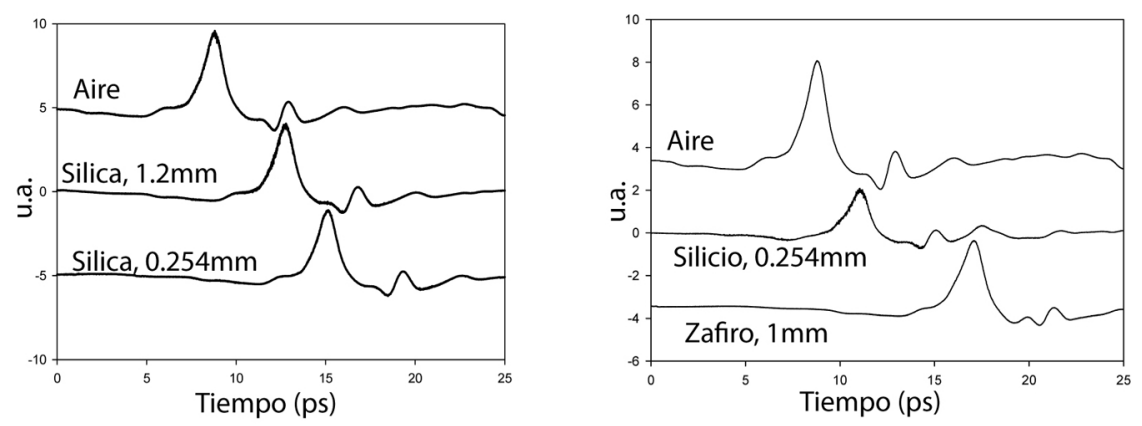

Figura 4.5: Resultados temporales.

siendo $E_{r 0}$ el valor máximo del campo eléctrico sin la muestra, y $E_{s 0}$ con la misma.

Midiendo temporalmente dos muestras del mismo material con diferentes espesores se elimina el coeficiente de Fresnel, obteniendo como absorbancia:

$$
\alpha=\frac{2}{\left(\mathrm{~d}_{2}-\mathrm{d}_{1}\right)} \ln \frac{E_{2 s 0}}{E_{1 s 0}} .
$$

\subsubsection{Resultados}

En la Tabla 4.1 se presentan los resultados de obtener los parámetros ópticos del zafiro, sílica, y silicio, mediante la información obtenida en el dominio temporal.

\begin{tabular}{|l|l|l|}
\hline Material & $\mathrm{n}$ & $\alpha\left(\mathrm{cm}^{-1}\right)$ \\
\hline Sílica & $1,98 \pm 0,02$ & $4,2 \pm 0,4$ \\
\hline Silicio & $3,56 \pm 0,14$ & $88 \pm 7$ \\
\hline Zafiro & $3,31 \pm 0,25$ & $18 \pm 4$ \\
\hline
\end{tabular}

Tabla 4.1: Resultados.

Para obtenerlos se utilizó el retardo y los picos de las señales mostrados en la Fig. 4.5 con el uso de las ecuaciones (4.9) y (4.10). Los resultados conseguidos también están de acuerdo con lo publicado en [63]. 


\section{3. $\quad$ Errores}

En la señal detectada se encuentran errores principalmente debido a las fuentes de ruido presentes en las antenas (shot noise y Johnson noise), y a la inestabilidad del láser (pink noise) [61, 65-67].

Para cuantificar los errores se obtuvo la varianza de los parámetros ópticos utilizando las ecuaciones publicadas en [61]. Las mismas, se obtienen de hacer propagación de errores a las ecuaciones (4.4) y (4.5):

$$
\begin{gathered}
\triangle n=\frac{c}{\omega \mathrm{d}} \sigma_{\theta(\omega)}+\frac{c}{\omega \mathrm{d}^{2}} \theta(\omega) \sigma_{d} \\
\triangle \alpha=\frac{2}{\mathrm{~d}}\left(\frac{(n-1)^{2}}{n\left(n^{2}-1\right)}\right) \triangle n+\frac{2}{\mathrm{~d}} \frac{\sigma_{T(\omega)}}{T(\omega)}+\frac{2}{\mathrm{~d}^{2}} \ln \left(\frac{(n+1)^{2}}{4 n} T(\omega)\right) \sigma_{d},
\end{gathered}
$$

donde d es el ancho de la muestra, $\theta(\omega)$ es la media de la fase, $\sigma_{\theta}$ es la varianza de la fase, $\sigma_{d}$ es la varianza de la medida del espesor, $T(\omega)$ es la media del módulo, y $\sigma_{T}$ es la varianza del módulo.

A partir del uso de dichas ecuaciones se realizó un programa en Matlab para determinar la dispersión en los datos obtenidos.

En la Figura 4.6 se presenta la varianza del índice de refracción y de la absorbancia de una oblea de silicio al hacer 15 medidas sobre la misma.

Al disminuir el tiempo entre medidas el error disminuía, con lo cual se infirió que la gran variación era causada por la inestabilidad del láser. 

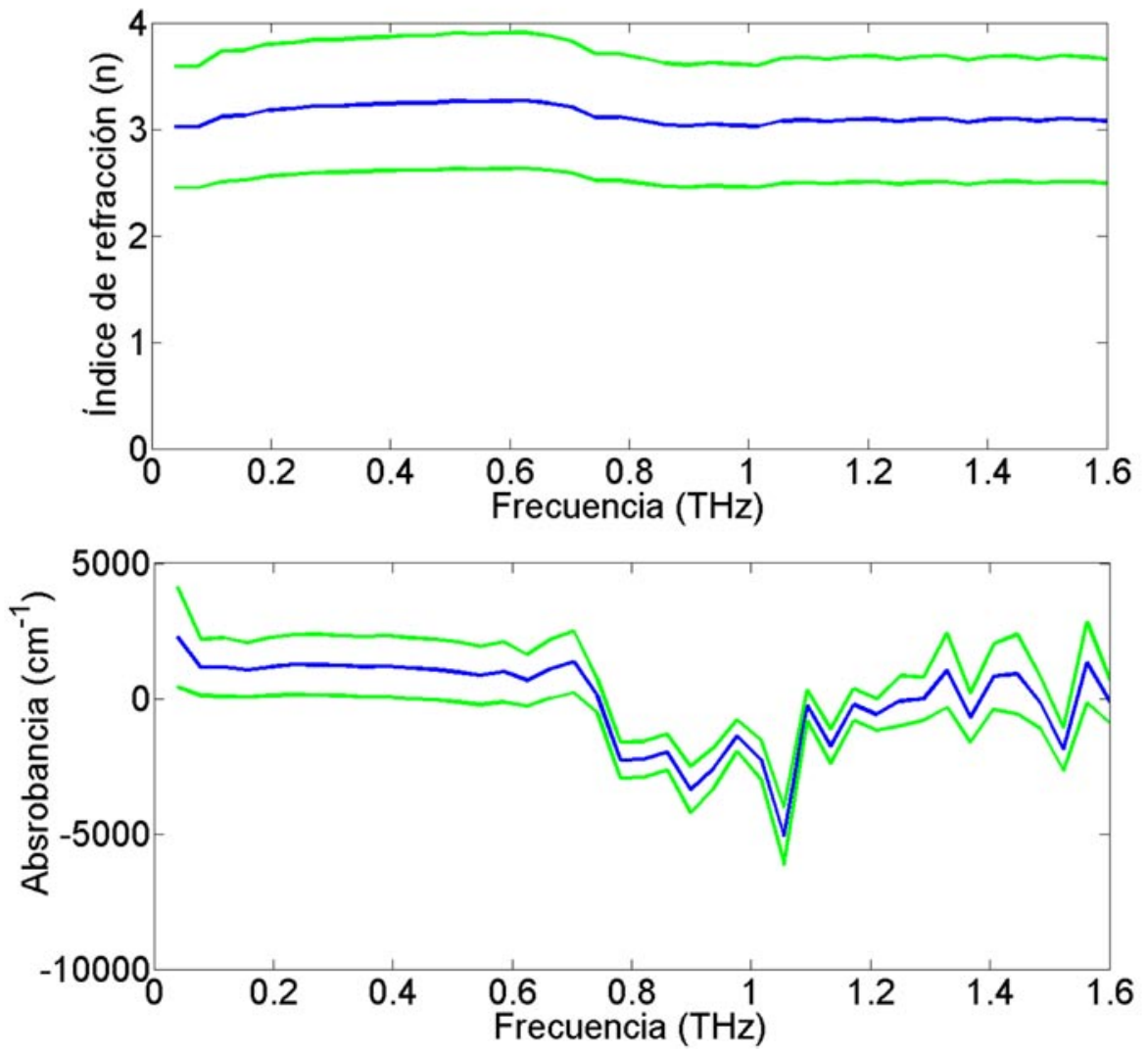

Figura 4.6: Varianza de los parámetros ópticos. 


\section{Capítulo 5}

\section{Método interferométrico de medición de materiales. Resultados}

\section{Resumen}

En cada una de las secciones del presente capítulo se encuentran desarrollados los trabajos publicados realizados durante una estancia en la ciudad de Valencia, España. La misma, tuvo lugar en el Centro de Tecnología Nanofotónica (NTC) de la Universidad Politécnica de Valencia (UPV) bajo la dirección del Dr. Borja Vidal.

Cada uno de los trabajos ha sido desarrollado haciendo uso de la información obtenida a través de la interferencia generada en los materiales bajo estudio. En el primer trabajo presentado se desarrolla una técnica para la obtención de los parámetros ópticos de un material monocapa [11] . En el segundo, se realiza un método para calcular los índices de refracción y posición del eje óptico en un material birrefringente [12]. En el último trabajo, se aplica el método a un material multicapa de manera de poder obtener en una sola medida los índices y los espesores de la estructura [13].

\subsection{Materiales monocapa}

\subsubsection{Resumen}

Se presenta un método que calcula el índice de refracción promedio de una muestra, utilizando una sola medida del espectrómetro de $\mathrm{THz}$. El índice de refracción es obtenido mediante un análisis de la interferencia presente en el 
dominio de la frecuencia, la cual es causada por las reflexiones Fabry-Pérot que se producen en la muestra. El método consiste en calcular el módulo a la transformada de Fourier discreta (DFT) del módulo del espectro. Dicho enfoque es simple y rápido (no necesita medida de referencia), mejora la sensibilidad respecto al análisis directo de la traza temporal, y permite la obtención del índice de refracción en un ancho de banda frecuencial específico. Asimismo, el método propuesto es útil para identificar reflexiones no deseadas en la configuración experimental.

\subsubsection{Introducción}

Usualmente el índice de refracción se consigue mediante la obtención de la función transferencia, como fue descripto en la Sección 4.1.1. Dicho método presenta como desventaja la necesidad de una medida de referencia, lo cual genera que la obtención del resultado sea mas lento y mas complejo de obtener. Dificultando por ejemplo el caso de una inspección online.

Otra manera usual de calcular el índice es mediante la información obtenida del retraso generado entre el pulso principal y los ecos [63]. La desventaja del método radica en que a veces es difícil distinguir el eco, debido a la atenuación que sufre y/o al ruido presente en la señal.

En el método propuesto [11] se mide el índice de refracción promedio utilizando una sola medida del espectrómetro de THz. A lo largo de la presente sección se muestra que aplicando el módulo de la transformada discreta de Fourier del módulo del espectro de la señal, se obtiene un resultado del índice mas tolerante al ruido, y que no necesita señal de referencia. Dicho método es válido para los casos en que el índice es prácticamente constante en el ancho de banda del espectrómetro.

\subsubsection{Método}

Un esquema en bloques del procesamiento de datos propuesto se muestra en la Fig. 5.1. En la misma se observa que la señal de entrada $I(t)$ consta de un pulso principal y un eco. También se visualiza que $X(f)$ presenta una modulación, la cual es producto de la diferencias de fase generadas entre las señales trasmitidas y reflejadas. Por último, $Y(t)$ representa la componente temporal responsable de dicha modulación. Con el uso del valor $k$ mostrado en la Fig. 5.1 se puede obtener el índice de refracción. En el caso en que haya múltiples ecos en $I(t)$, $Y(t)$ presentaría armónicos del valor $k$ decreciendo en amplitud. 


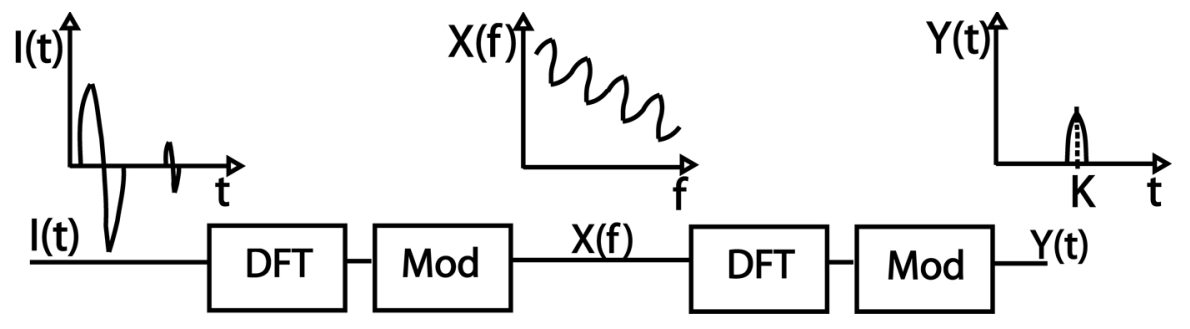

Figura 5.1: Diagrama en bloques del procesamiento de datos.

\subsubsection{Teoría}

Por simplicidad en el desarrollo se considera para el análisis teórico señales continuas. El espectro del campo eléctrico a la salida de la muestra puede escribirse como [61]:

$$
E_{s}=\left|E_{i}(\omega)\right| e^{j \theta_{i}(\omega)} \frac{4}{(n(\omega)+1)^{2}} e^{-j n(\omega) \omega \mathrm{d} / \mathrm{c}} \sum_{m=0}^{\infty}\left(\frac{(n(\omega)-1)^{2}}{(n(\omega)+1)^{2}} e^{-j 2 n(\omega) \omega \mathrm{d} / \mathrm{c}}\right)^{m},
$$

siendo $\left|E_{i}(\omega)\right| e^{j \theta_{i}(\omega)}$ el campo eléctrico incidente, $c$ la velocidad de la luz, $d$ el ancho de la muestra, $n$ el índice de refracción, y $m$ un número entero que representa la cantidad de ecos producidos por las reflexiones en el material.

Asumiendo que los índices de refracción y $\left|E_{i}(\omega)\right|$ son constantes en frecuencia, los pasos mostrados en la Fig. 5.1 aplicados a la ecuación (5.1) generan lo siguiente:

$$
Y(t)=C_{0} \delta(0)+\sum_{m=1}^{\infty} C_{m}\left(\delta\left(t-\frac{2 n d}{c} m\right)+\delta\left(t+\frac{2 n d}{c} m\right)\right) .
$$

Los coeficientes $C_{m}$ son constantes que dependen del índice de refracción y de la amplitud del campo eléctrico. Las deltas de Dirac tienen un desplazamiento dado por $k= \pm 2 n d m / c$ que son consecuencia de la modulación provocada por la interferencia (ver Fig. 5.1). Con dicho valor podemos escribir el índice de refracción como:

$$
n=\frac{c k}{2 d m}, m=1,2,3, \ldots
$$

Debido a que la señal es finita y a que puede haber variaciones del índice de refracción en el rango frecuencial de interés, $Y(t)$ deja de ser una delta para convertirse en un pulso temporal de cierto ancho. En el caso particular en que el índice de refracción del material varíe mucho en el rango frecuencial, se obtiene un índice de refracción promedio. El cual puede ser útil cuando se necesita un 
valor inicial en los casos de cálculo de índice de refracción de modo iterativo [60]. El error de la medida se estima realizando propagación de errores a la ecuación (5.3), considerando el índice de refracción independiente de la frecuencia.

$$
\triangle E_{n}=\frac{k}{2 d m} \triangle E_{c}+\frac{c}{2 d m} \triangle E_{T}+\frac{c k}{2 d^{2} m} \triangle E_{d}
$$

Donde $\triangle E_{c}$ es el error debido al valor aproximado de la velocidad de la luz, $\triangle E_{T}$ es el error absoluto causado por la resolución temporal del espectrómetro, y $\triangle E_{d}$ es el error en la medida del espesor de la muestra. Cabe mencionar que usualmente la mayor causa de error esta dada por $\triangle E_{d}$. En la ecuación (5.4) se observa que en casos de muestras de mayor espesor el error decrece. Esto se debe a que al aumentar $d$, disminuye sobretodo el tercer término que es el responsable del mayor error.

\subsubsection{Experimento y resultados}

El experimento se realizó mediante el uso de un láser mode-locked modelo FemtoFErb 1560 de la empresa Toptica y antenas fotoconductoras TERA15SL25-FC y TERA15-DP25-FC de la empesa Menlo Systems. Las medidas se realizaron con un barrido temporal de 232 ps, y con una resolución temporal de 14.15 fs. Se utilizaron muestras de sílica de 1.21, 1.97 y $3.1 \mathrm{~mm}$ de espesor.

En la Figura 5.2(a) se presenta el pulso temporal medido con la muestra de $1.21 \mathrm{~mm}$ de espesor, donde se observa que los ecos no son claramente visibles. En la Figura 5.2(b) se calcula el valor absoluto de la DFT de la Fig. 5.2(a), en la cual se puede evidenciar la modulación causada por la interferencia del pulso principal con sus ecos. La Figura 5.2(c) muestra el resultado final del procesamiento de las 3 muestras que estuvieron bajo estudio. Los valores de los índices de refracción obtenidos están de acuerdo con los valores presentados en la referencia [63].

En el análisis espectral de la Fig. 5.2(c) se observan otras componentes aparte de las causadas por el pulso principal con los ecos. En el caso de considerar la muestra de $1.21 \mathrm{~mm}$, cualquier pico debajo de los 8 ps correspondería a un retraso temporal provocado por un material con índice de refracción menor a uno. Por tal motivo se consideró el pico que se encontraba luego de los 8 ps marcado como $k_{1}$ en la Fig. 5.2(c) para el cálculo del índice de refracción.

El origen de los picos por debajo de los 8 ps son generados por reflexiones no deseadas del espectrómetro. Por tal, no dependen de la muestra bajo estudio. Tales picos que no son visualizados en la Fig. 5.2(a) pueden ser utilizados para encontrar las fuentes que generan reflexiones de manera de mejorar el funcionamiento del espectrómetro. En el caso de la configuración experimental utilizada el resultado obtenido apunta a reflexiones ocasionadas por espacios de aire de 


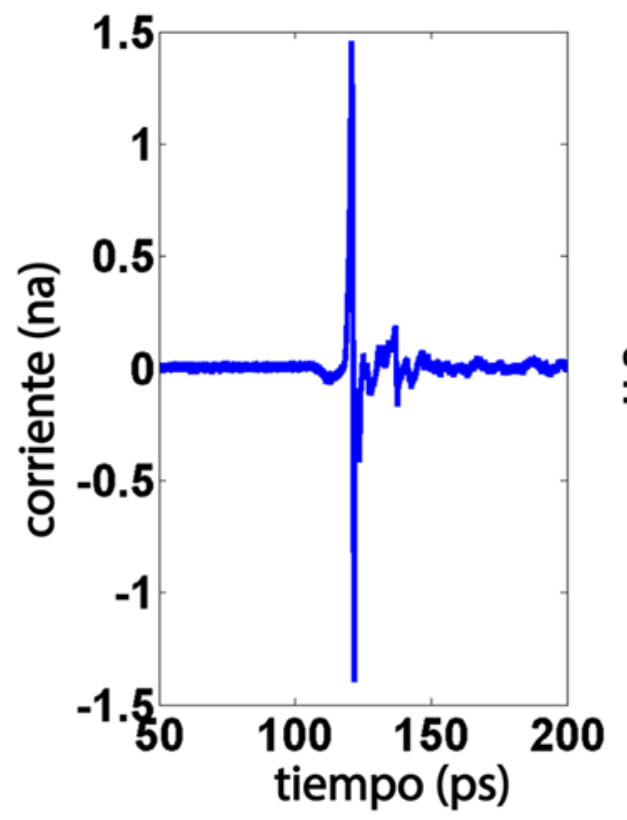

(a)

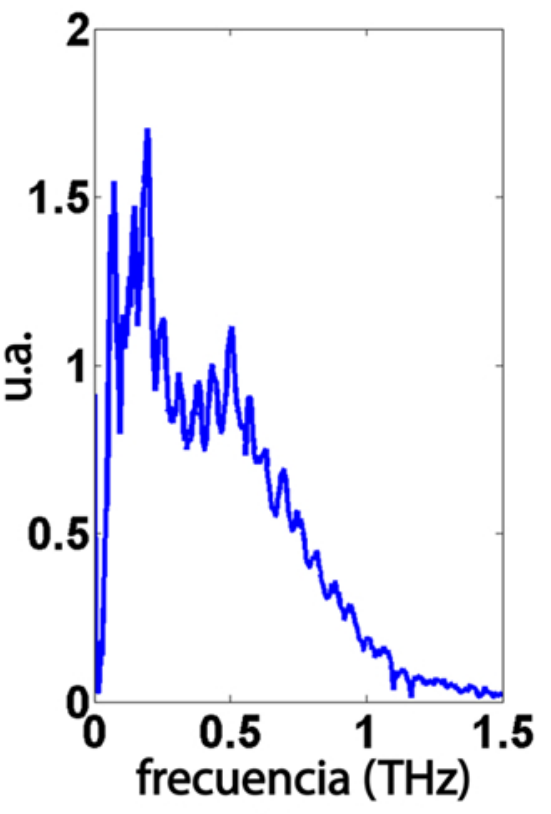

(b)

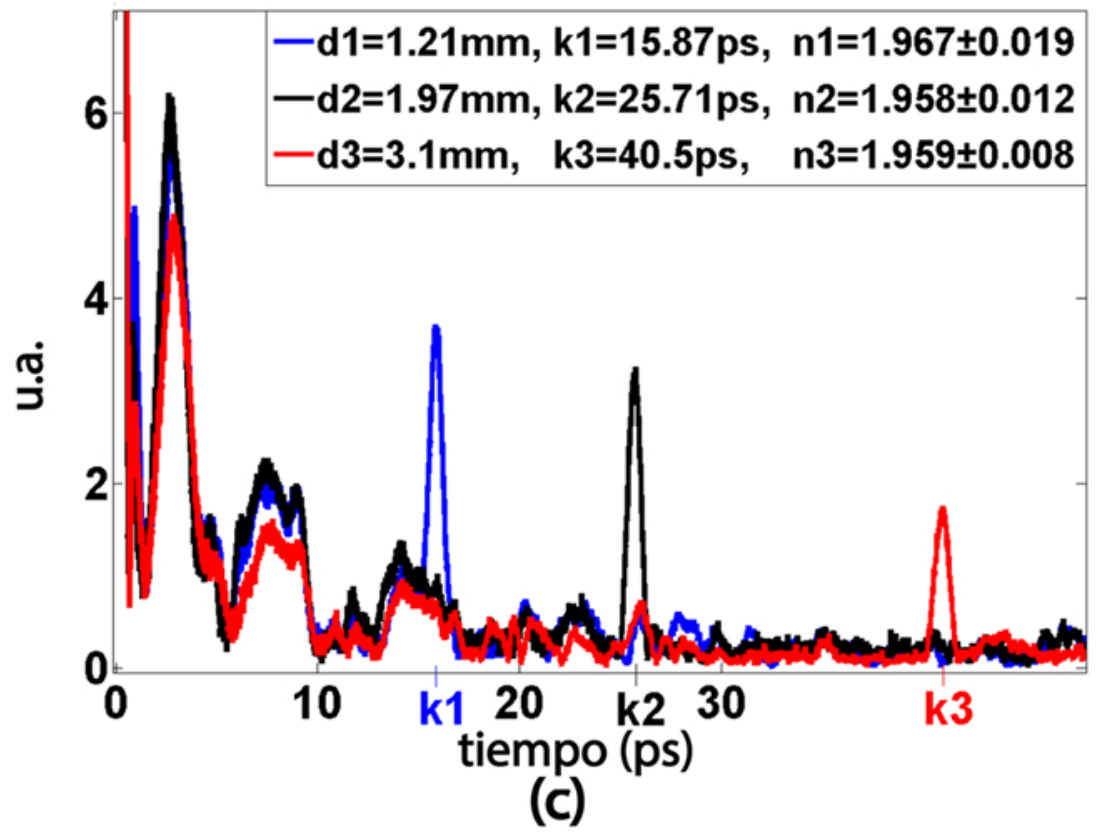

Figura 5.2: Medidas en muestras de sílica. (a) Señal temporal del espectrómetro de la muestra de sílica de $1.21 \mathrm{~mm}$. (b) Espectro de la señal luego de realizar el valor absoluto de la DFT de la Fig. 5.2(a). (c) La línea azul corresponde al análisis espectral de la Fig. 5.2(b); Las líneas negra y roja son el resultado final del procesamiento en las muestras de $1.97 \mathrm{~mm}$, y $3.1 \mathrm{~mm}$ respectivamente. 
400 y $140 \mu \mathrm{m}$. Éstas probablemente se encuentren en la unión de la antena con la lente de silicio.

La mejora en la discriminación sobre la observación directa en el dominio temporal de la señal es generada porque las componentes de ruido aleatorio no producen modulación en frecuencia apreciable. De esa manera es posible detectar una interferencia que produzca una componente frecuencial pequeña.

Una variante del método propuesto es procesar los datos sin considerar todo el ancho de banda del espectrómetro, con lo cual se puede seleccionar la ventana de frecuencias que sea de interés. Tal acción es útil cuando el índice es constante en cierto rango de frecuencias, o cuando se prefiera seleccionar la región que presente el mejor rango dinámico. Tal posibilidad no es aplicable sobre un análisis directo de los datos temporales medidos.

\subsubsection{Conclusión}

Se propuso un método que calcula el índice de refracción mediante el uso de una sola medición del espectrómetro de $\mathrm{THz}$, sin necesidad de tener una medida de referencia. Con el mismo se obtiene buenos resultados, aún cuando los ecos están inmersos en ruido, o cuando hay señales no deseadas presentes. También, el método permite la determinación del índice de refacción promedio en una ventana de frecuencias, e incluso posibilita identificar reflexiones no deseadas provocadas por la configuración experimental del espectrómetro.

\subsection{Materiales birrefringentes}

\subsubsection{Resumen}

Se desarrolló un método para la obtención de los índices de refracción de un material birrefringente en la banda de los terahertz, mediante el uso de una sola medida del espectrómetro. El método se basa en la utilización de la información suministrada por la interferencia generada por los pulsos principales con sus respectivos ecos. La técnica también permite la determinación de la posición del eje óptico mediante dos medidas variando el ángulo del mismo.

\subsubsection{Introducción}

Medir la birrefringencia puede ser útil para obtener parámetros como el estrés mecánico de los plásticos, alineación de las cadenas moleculares en cristales líquidos, orientación de las fibras del papel, materiales compuestos, maderas y otros materiales [68-70]. 
Para la medición de birrefringencia se han desarrollado técnicas en el visible, en el infrarrojo cercano (NIR), por difracción de rayos x (XRD), y mediante el uso de radiación de $\mathrm{THz}$.

La ventaja de la espectroscopía de $\mathrm{THz}$ radica en que permite caracterizar materiales de interés industrial como plásticos, madera, textiles y papel que son opacos en el visible y NIR, con lo cual se logra un análisis a mayor profundidad del material. También ofrece un enfoque mas seguro ya que no es ionizante como el caso de XRD [68].

La birrenfringencia del material, así como la posición del eje óptico en la banda de $\mathrm{THz}$ han sido obtenidos de diferentes maneras. El procedimiento más común basado en el uso de un espectrómetro de $\mathrm{THz}$ resuelto en el tiempo, consiste en utilizar la información obtenida de una medida de referencia y otras tres variando el ángulo de la muestra [71, 72]. Dicho método presenta como desventaja ser un procedimiento lento de medición, por el hecho de realizar cuatro medidas cambiando las condiciones del experimento.

Otra propuesta, es obtener los índices de refracción promedio midiendo el retraso temporal entre los picos de los pulsos principales, con sus respectivos ecos [73]. Tal procedimiento sólo es posible si el retraso entre los pulsos es distinguible por el espectrómetro. Por ejemplo, cuando el retraso entre los pulsos es del orden de 1 ps, no se puede aplicar dicho método. También, haciendo uso de la información de los pulsos temporales medidos se puede determinar la posición del eje óptico. El mismo, se obtiene relacionando las amplitudes pico de los dos pulsos principales [68]. Tal enfoque, da un mal resultado cuando el material presenta diferente atenuación en cada eje del material.

En la referencia [74] se describe un método para determinar los índices de refracción y la posición del eje óptico en una sola medida. La técnica utiliza un espectrómetro de $\mathrm{THz}$ que consiste en un emisor con polarización circular, y un detector sensible a la polarización que mide ambas componentes del campo eléctrico simultáneamente. Presenta como desventaja, no poder implementarse con un espectrómetro de $\mathrm{THz}$ estándar.

En el trabajo realizado se propuso una técnica para medir los índices de refracción promedios y la posición del eje óptico de un material birrefringente. Tal propuesta es válida para materiales con índice constante en el rango de los THz, como es el caso de los plásticos, papeles y maderas [70-73]. El método se basa en el uso de la información generada por la interferencia provocada por los pulsos con los ecos en el dominio de la frecuencia. En particular, el método al igual que el caso presentado en la Sección 5.1, se basa en aplicar el módulo a la transformada discreta de Fourier del módulo al cuadrado del espectro de la señal detectada. 


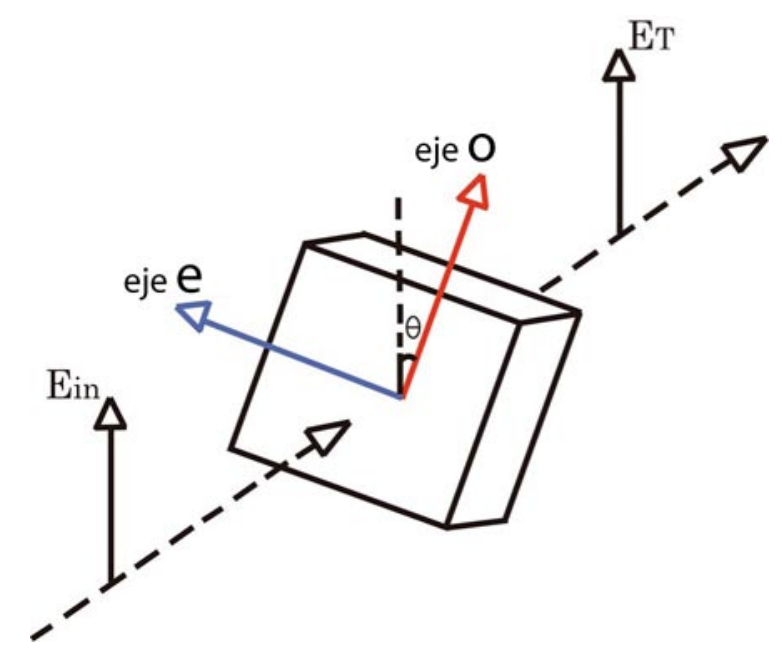

Figura 5.3: Proyección del campo eléctrico de THz sobre el material birrefringente.

\subsubsection{Teoría y simulación}

La antena fotoconductora emite con polarización lineal y el detector es sensible a la misma. Por tal motivo se muestra en la Fig. 5.3 la interacción del campo eléctrico linealmente polarizado sobre una muestra birrefringente. El campo eléctrico proveniente del generador es proyectado sobre el eje ordinario y extraordinario, siendo afectado con diferentes retardos y atenuaciones. En el detector, ambas componentes vuelven a ser proyectadas en la dirección de polarización en la que es sensible el mismo. Considerando por simplicidad que la señal es continua, el campo eléctrico a la salida del material en el dominio de la frecuencia puede escribirse como $[61,71]$ :

$$
\begin{aligned}
E_{T}(\omega)= & \left|E_{i}(\omega)\right| \cos ^{2}(\theta) e^{j \phi_{i}(\omega)} T_{0}(\omega) G_{0}(\omega, d) \sum_{m=0}^{\infty}\left(r_{0}(\omega) G_{0}^{2}(\omega, d)\right)^{m} \\
& +\left|E_{i}(\omega)\right| \operatorname{sen}^{2}(\theta) e^{j \phi_{i}(\omega)} T_{e}(\omega) G_{e}(\omega, d) \sum_{m=0}^{\infty}\left(r_{e}(\omega) G_{e}^{2}(\omega, d)\right)^{m}
\end{aligned}
$$

donde $\left|E_{i}(\omega)\right| e^{j \phi_{i}(\omega)}$ es el campo eléctrico incidente, $T(\omega)=\frac{4 n(\omega)}{(n(\omega)+1)^{2}}, \mathrm{y}$ $r(\omega)=\frac{(n(\omega)-1)^{2}}{(n(\omega)+1)^{2}}$, son el cuadrado del coeficiente de transmisión y reflexión respectivamente; $G(\omega)=e^{-j n(\omega) \omega d / c}$ es el coeficiente de propagación, $\omega$ es la frecuencia angular; $\theta$ es el ángulo entre el eje óptico de la muestra y el eje de polarización lineal de la antena; $d$ es el espesor de la muestra, y $m$ es el número de ecos debido al efecto Fabry-Pérot.

En la ecuación (5.5) el primer término es afectado por el índice de refracción ordinario $\left(n_{0}\right)$, y el segundo por el extraordinario $\left(n_{e}\right)$. 
La ecuación (5.6) se obtiene al realizar el módulo al cuadrado de (5.5). Se asume los índices de refracción constantes en frecuencia, y se considera un solo eco en cada término ya que los siguientes son mucho menores en amplitud. También se asume $\left|E_{i}(\omega)\right|^{2}$ constante en frecuencia.

$$
\begin{aligned}
\left|E_{T}(\omega)\right|^{2}= & \left.a_{1}\left(\theta, n_{e}, n_{0}\right)\right)\left|E_{i}(\omega)\right|^{2} \cos \left(\left(n_{0}-n_{e}\right) \frac{\omega d}{c}\right. \\
& \left.+a_{2}\left(\theta, n_{e}, n_{0}\right)\right)\left|E_{i}(\omega)\right|^{2} \cos \left(\left(3 n_{0}-n_{e}\right) \frac{\omega d}{c}\right. \\
& \left.+a_{3}\left(\theta, n_{e}, n_{0}\right)\right)\left|E_{i}(\omega)\right|^{2} \cos \left(\left(n_{0}-3 n_{e}\right) \frac{\omega d}{c}\right. \\
& \left.+a_{4}\left(\theta, n_{e}, n_{0}\right)\right)\left|E_{i}(\omega)\right|^{2} \cos 2 n_{0} \frac{\omega d}{c} \\
& \left.+a_{5}\left(\theta, n_{e}, n_{0}\right)\right)\left|E_{i}(\omega)\right|^{2} \cos 2 n_{e} \frac{\omega d}{c} \\
& \left.+a_{6}\left(\theta, n_{e}, n_{0}\right)\right)\left|E_{i}(\omega)\right|^{2} \cos \left(3\left(n_{0}-n_{e}\right) \frac{\omega d}{c}\right. \\
& \left.+a_{7}\left(\theta, n_{e}, n_{0}\right)\right)\left|E_{i}(\omega)\right|^{2} .
\end{aligned}
$$

Aplicando la transformada de Fourier a (5.6) se obtiene la ecuación (5.7). En la misma, se observa que aparte de la componente continua hay seis términos presentes en el receptor.

$$
\begin{aligned}
Y_{T}(t)= & H_{1}\left(\theta, n_{e}, n_{o}\right) \delta\left(t-\left|n_{o}-n_{e}\right| \frac{d}{c}\right) \\
& +H_{2}\left(\theta, n_{e}, n_{o}\right) \delta\left(t-\left|3 n_{o}-n_{e}\right| \frac{d}{c}\right) \\
& +H_{3}\left(\theta, n_{e}, n_{o}\right) \delta\left(t-\left|n_{o}-3 n_{e}\right| \frac{d}{c}\right) \\
& +H_{4}\left(\theta, n_{e}, n_{o}\right) \delta\left(t-2 n_{o} \frac{d}{c}\right) \\
& +H_{5}\left(\theta, n_{e}, n_{o}\right) \delta\left(t-2 n_{e} \frac{d}{c}\right) \\
& +H_{6}\left(\theta, n_{e}, n_{o}\right) \delta\left(t-3\left|n_{o}-n_{e}\right| \frac{d}{c}\right) \\
& +H_{7}\left(\theta, n_{e}, n_{o}\right) \delta(0) .
\end{aligned}
$$

La Figura 5.4 muestra una simulación numérica del valor absoluto de la DFT de la ecuación (5.6), asumiendo un ancho de banda de $3 \mathrm{THz}$, una resolución espectral de $5 \mathrm{GHz}, n_{e}=3,07, n_{o}=3,39, d=1 \mathrm{~mm}$, y $\theta=45^{\circ}$. 


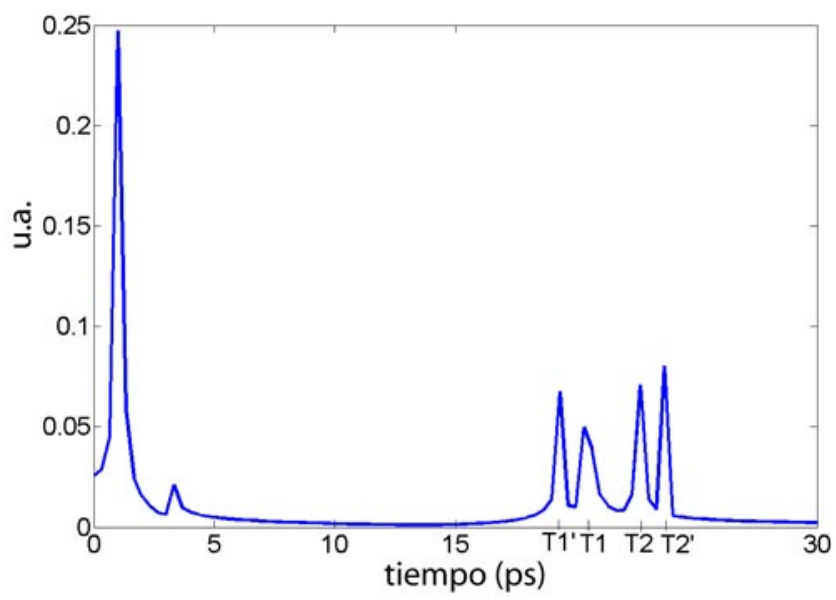

Figura 5.4: Simulación numérica de la DFT aplicada a la señal frecuencial de la ecuación (5.6).

Debido a que la señal es finita, los picos que se observan en la Fig. 5.4 no son deltas. También cabe mencionar que los picos que están cerca del origen corresponden al primer y sexto término de la ecuación (5.7). En particular, los picos utilizados para el cálculo de los índices son los suministrados por el cuarto y quinto término de la ecuación (5.7), que corresponden a los desplazamientos temporales $T_{1}=2 n_{o} d / c$, y $T_{2}=2 n_{e} d / c$ (ver Fig. 5.4). Los mismos son útiles para obtener los índices haciendo $n_{o}=T_{1} c / 2 d$ y $n_{e}=T_{2} c / 2 d$.

En la práctica, cuando el valor $n_{o}$ es similar a $n_{e}$, el segundo y cuarto término de la ecuación (5.7) tienen un desplazamiento temporal muy similar. Lo mismo ocurre con el tercer y quinto término. Tal situación, genera que dependiendo de la resolución del espectrómetro es posible que se distingan dos picos en vez de los cuatro presentados en la Fig. $5.4\left(T_{1}, T_{1}^{\prime}, T_{2}, T_{2}^{\prime}\right)$.

En dicho caso se aproxima el resultado considerando el desplazamiento temporal $T_{1}=2 n_{o} d / c$ igual $T_{1}^{\prime}=\left(3 n_{o}-n_{e}\right) d / c$, y lo mismo con $T_{2}=2 n_{e} d / c$ y $T_{2}^{\prime}=\left(3 n_{e}-n_{o}\right) d / c$. Esto genera que el resultado del índice de refracción sea calculado como se describió anteriormente utilizando $T_{1}$ y $T_{2}$, pero considerando un error mayor, como se describe mas adelante.

Para estimar el error en el cálculo del índice de refacción se realiza la propagación de errores a $n=T c / 2 d$, obteniendo:

$$
\triangle E_{n}=\frac{T}{2 d m} \triangle E_{c}+\frac{c}{2 d m} \triangle E_{T}+\frac{c T}{2 d^{2} m} \triangle E_{d}
$$

Donde $\triangle E_{c}$ es el error debido a la aproximación de la velocidad de la luz, $\triangle E_{d}$ es el error en la medida de la muestra, y $\triangle E_{T}$ es el error causado por la 
resolución obtenida en la última DFT aplicada, la cual depende del ancho de banda del espectrómetro.

En el caso en que se observen dos picos el error debido a la resolución temporal aumenta. Esto ocurre porque puede medirse $T_{1}$ en vez de $T_{1}^{\prime}$, o $T_{2}$ en vez de $T_{2}^{\prime}$ (ver Fig. 5.4). Por lo tanto, el error debido a la resolución temporal puede escribirse como: $\triangle E_{T}^{\prime}=\triangle E_{T}+\left|T_{1}^{\prime}-T_{1}\right|=\triangle E_{T}+\left|n_{o}-n_{e}\right| d / c=$ $\triangle E_{T}+\left|T_{1}-T_{2}\right| / 2$. En la simulación numérica mostrada en la Fig. 5.4 el error en el índice de refracción utilizando la ecuación (5.8) fue de 0,08.

A continuación se muestra como calcular la posición del eje óptico con la información de la ecuación (5.7). Haciendo el cociente de las amplitudes del cuarto y quinto término se obtiene:

$$
\frac{H_{4}}{H_{5}}=\operatorname{tg}^{4} \theta \frac{\left(\frac{4 n_{e}}{\left(n_{e}+1\right)^{2}}\right)^{2} \frac{\left(n_{e}-1\right)^{2}}{\left(n_{e}+1\right)^{2}}}{\left(\frac{4 n_{o}}{\left(n_{o}+1\right)^{2}}\right)^{2} \frac{\left(n_{o}-1\right)^{2}}{\left(n_{o}+1\right)^{2}}}
$$

Si el valor $n_{o}$ es similar a $n_{e}$, la ecuación (5.9) se puede aproximar por:

$$
\theta \approx t g^{-1}\left(\left(\frac{H_{4}}{H_{5}}\right)^{1 / 4}\right)
$$

Para determinar si el ángulo obtenido del eje óptico se encuentra a sentido horario o antihorario, hay que realizar otra medida con un diferente ángulo de la muestra.

En el caso en que se pueda discernir solo dos picos, la posición del eje óptico es calculado haciendo el cociente de la suma de los términos que tienen el desplazamiento temporal mas cercano. Obteniendo:

$$
\frac{H_{4}+H_{2}}{H_{5}+H_{3}}=t g^{2} \theta f\left(n_{o}, n_{e}, \theta\right)
$$

Cuando $n_{o}$ tiene un valor cercano a $n_{e}, f\left(n_{o}, n_{e}, \theta\right)$ se puede aproximar a uno consiguiendo el siguiente resultado:

$$
\theta \approx t g^{-1}\left(\frac{H_{4}+H_{2}}{H_{5}+H_{3}}\right)^{1 / 2} .
$$

En la Figura 5.5 se presenta una simulación de la DFT de la ecuación (5.6), asumiendo un ancho de banda de $1 \mathrm{THz}$, una resolución espectral de $2.94 \mathrm{GHz}$, $n_{e}=3,07, n_{o}=3,39, d=1 \mathrm{~mm}$ y $\theta$ variando en $49.8^{\circ}, 54.6^{\circ}$, y $58.8^{\circ}$. En la figura se observan solo dos picos debido a la disminución del ancho de banda y de la resolución espectral respecto a la utilizada en la Fig. 5.4. 


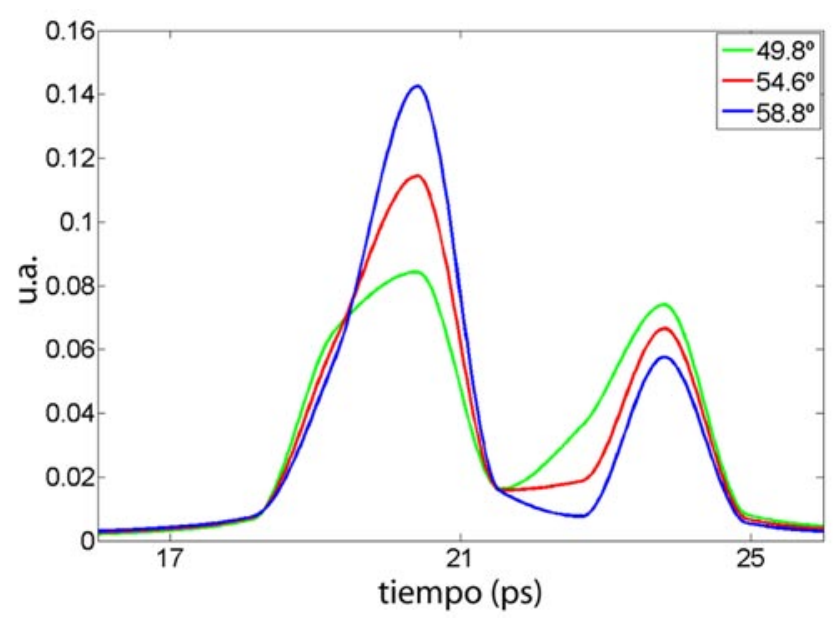

Figura 5.5: Simulación numérica de la DFT aplicada a la señal de la ecuación (5.6) para distintos ángulos de la muestra.

\subsubsection{Experimento y resultados}

Para validar el método propuesto se realizaron medidas con el espectrómetro montado en el Centro de Tecnología Nanofótonica de la Universidad Politécnica de Valencia. Dicho instrumento consiste principalmente en un láser de femtosegundos mode-locked modelo FemtoFErb 1560 de la empresa Toptica y antenas fotoconductoras TERA15-SL25-FC y TERA15-DP25-FC de la empesa Menlo Systems, y un amplificador de lock-in para adquirir los datos. Cabe mencionar, que la propagación de la luz estaba confinada en fibra óptica como se muestra en la Fig. 5.6, donde se presenta la configuración experimental.

En el experimento se utilizó una muestra de zafiro de $1 \mathrm{~mm}$ de espesor, la cual presenta una diferencia entre el índice ordinario y extraordinario de 0.32 [72]. Tal variación de índices produce un retraso entre los pulsos principales de 1 ps. Esto evidencia que con un análisis directo en el dominio temporal no puede resolverse el cálculo de los mismos. Por tal motivo en la Fig. 5.7(a) se observa un sólo pulso principal con su eco, en vez de dos pulsos principales con sus respectivos ecos.

La medida fue realizada con un barrido temporal de $340 \mathrm{ps}$, con pasos de 200 fs. La Figura 5.7(b) muestra el resultado de hacer el módulo al cuadrado de la DFT de la señal temporal de la Fig. 5.7(a). En la misma se visualiza la modulación producida por la interferencia. Finalmente, en la Fig. 5.7(c) se muestra el módulo de la DFT de la señal de la Fig. 5.7(b). En la misma se observan sólo dos picos debido al ancho de banda limitado del espectrómetro. No obstante, se calcularon los índices del material con los valores mostrados en la figura. Con $T_{1}=20,2 \mathrm{ps}$, se obtuvo $n_{1}=3,03$, y con $T_{2}=23,31 \mathrm{ps}, n_{2}=3,49$. 


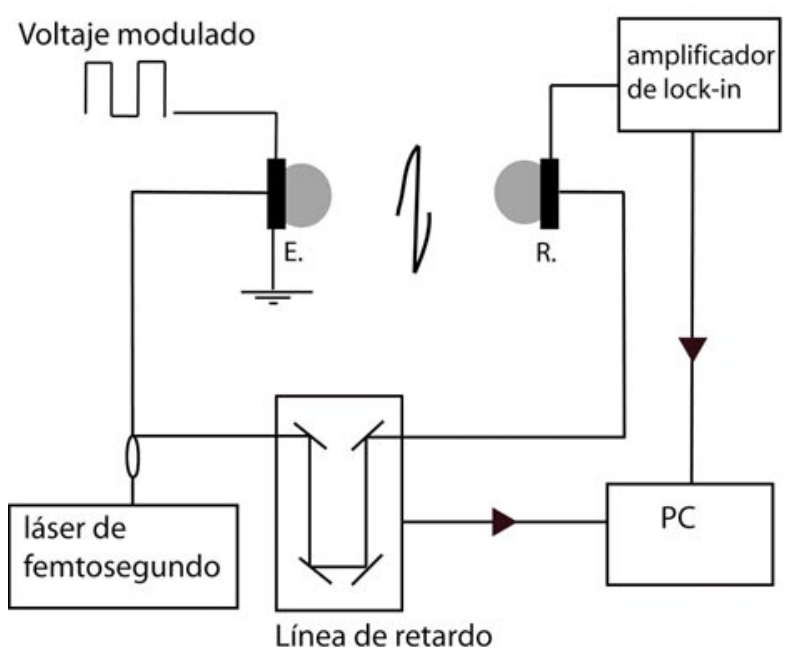

Figura 5.6: Configuración experimental del espectrómetro de THZ.
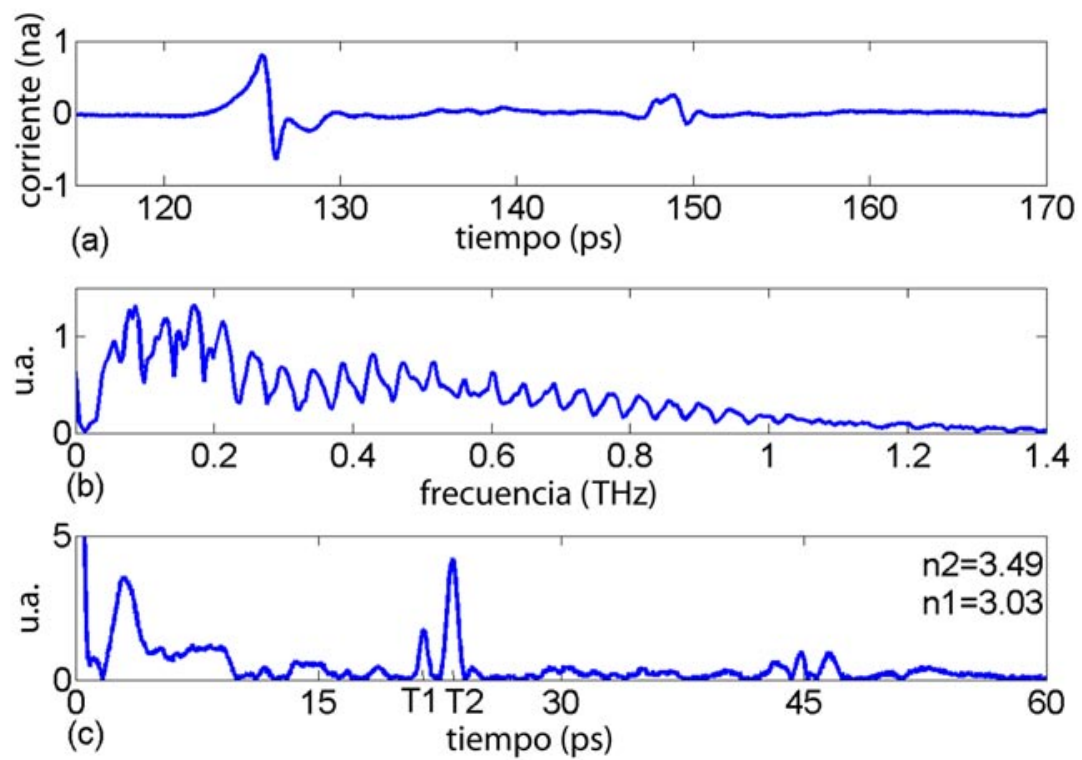

Figura 5.7: (a) Medición temporal del espectrómetro de THz con una ventana de zafiro de $1 \mathrm{~mm}$ de espesor. (b) Es el módulo de la DFT aplicada a la señal en (a). (c) Es el módulo al cuadrado de la DFT de la señal en (b). 


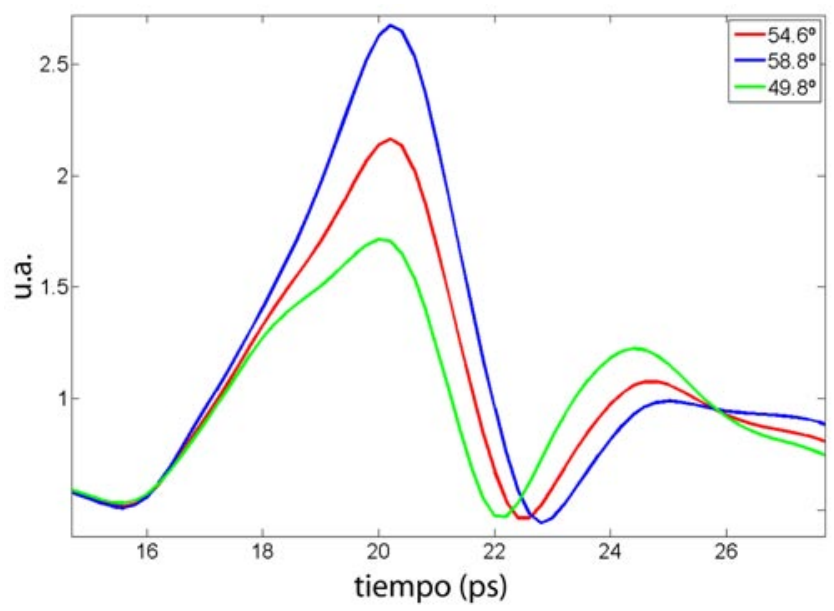

Figura 5.8: Medidas de la DFT del módulo del espectro rotando la muestra cada 5 grados.

Dichos valores de los índices están de acuerdo con lo publicado [63, 72].

En la Figura 5.7(c) se observan picos cerca del origen que son causados por reflexiones de la configuración experimental. También se observan armónicos cerca de los 45 ps de baja amplitud correspondientes a la interferencia generada por la próxima serie de ecos $(m=2)$.

El retraso entre los pulsos mostrados en la Fig. 5.7(c) está dado por, $\triangle t_{2}=$ $T_{2}-T_{1}=\left(2 n_{2}-2 n_{1}\right) d / c=2\left(n_{2}-n_{1}\right) d / c$, que resulta igual a $2 \triangle t_{1}$. Este resultado evidencia que la resolución en comparación con un análisis temporal directo, mejora en un factor 2.

También, se realizaron experimentos para lograr determinar la posición del eje óptico del material. En la Figura 5.8 se presentan 3 medidas realizadas rotando el ángulo de la muestra cada cinco grados en cada una.

Utilizando los picos de las amplitudes se calcularon los ángulos con el uso de la ecuación (5.12). Se obtuvo que el resultado coincidía con la rotación angular realizada sobre la muestra con un error máximo de $1^{\circ}$.

En la Figura 5.5 se realizó una simulación con la misma resolución, ángulos, espesor del material, y índice de refracción que en el caso experimental. Como resultado se observa que tanto la simulación, como el resultado experimental de la Fig. 5.8 son muy parecidos. Las pequeñas diferencias son causadas por asumir que los índices son independientes de la frecuencia, al igual que el campo eléctrico incidente en la ecuación (5.6). 


\subsubsection{Conclusión}

Se propuso un método simple para caracterizar materiales birrefringentes, con el uso de un espectrómetro estándar de THz. Permite el cálculo de los índices de refracción y la posición del eje óptico, incluso cuando hay diferentes atenuaciones en el eje ordinario y extraordinario del material.

El método permite una rápida caracterización de materiales transparentes, como plásticos, papel, y madera, ya que no necesita medida de referencia.

\subsection{Materiales multicapa}

\subsubsection{Resumen}

En este trabajo se realizó un procesamiento de datos para determinar los índices promedios de refracción, y espesores de una estructura bicapa. El método obtiene los parámetros utilizando la información de una sola medida de un espectrómetro de $\mathrm{THz}$ estándar, y mediante el conocimiento del espesor total de la estructura. La técnica subyace en el uso de la información causada por la interferencia generada entre los pulsos principales con sus respectivos ecos. Asimismo, el método permite hacer un estudio no invasivo de la estructura bicapa.

\subsubsection{Introducción}

El uso de los $\mathrm{THz}$ para medir estructuras multicapa tiene como ventaja frente a los métodos que trabajan en la región óptica [75], que puede medir materiales que son opacos en la misma. Asimismo, al ser una radiación no ionizante tiene ventajas frente a los análisis realizados con rayos $\mathrm{X}[76]$.

En la banda de $\mathrm{THz}$ primeramente se ha desarrollado un método que utiliza la matriz transferencia para analizar estructuras multicapa, con el cual se logra obtener parámetros de una capa inmersa en dos capas conocidas [77, 78].

En el caso de querer obtener todos los parámetros de la estructura, existe un método que utiliza las relaciones entre la señal trasmitida con una muestra de referencia a incidencia normal, y señales trasmitidas en la muestra de interés a ángulos oblicuos de incidencia [79]. Dicho enfoque es similar a espectroscopía angular elipsilométrica (angle spectroscopic ellipsometry). La desventaja del método esta presente en la complejidad del procesamiento, y en la configuración experimental.

Otro método propuesto descripto en al referencia [80], se basa en un algoritmo que en vez de ajustar el modelo teórico con la función transferencia experimental en el dominio de la frecuencia como en [79], utiliza el dominio 


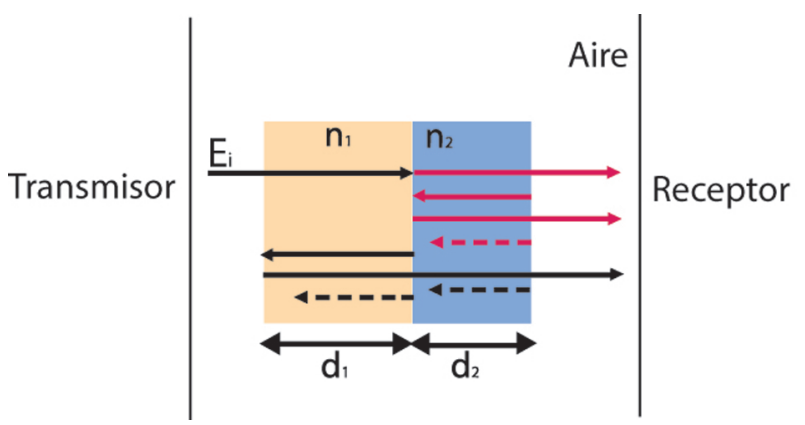

Figura 5.9: Traza de rayos del campo eléctrico en una estructura bicapa.

temporal. No obstante, cabe mencionar que necesita medición simultánea de la señal por reflexión y por transmisión.

En el trabajo desarrollado se realizó un procesamiento de datos que extrae tanto el espesor de las capas de la muestra, como los índices promedio de una estructura bicapa. La técnica se basa en el procesamiento de la interferencia generada en el material como se ha descripto en los trabajos publicados anteriormente $[11,12]$, aplicada a la problemática particular de una estructura bicapa.

\subsubsection{Teoría y simulación}

El procesamiento de datos se basa en realizar el módulo de la transformada de Fourier del módulo cuadrado del espectro.

El enfoque teórico se realiza asumiendo incidencia normal del campo eléctrico en la muestra, y considerando solamente las señales trasmitidas mostradas en la Fig. 5.9. El mismo análisis es válido en el caso en que las medidas estén realizadas por reflexión.

Para el análisis teórico se considera sólo la generación de un eco en cada material, y a los rayos reflejados representados con líneas punteadas (ver Fig. 5.9) se los desprecia por su baja amplitud respecto a las demás señales.

Las componentes del campo eléctrico en el dominio de la frecuencia luego del primer material, se puede escribir como:

$$
E_{t 1}(\omega)=E_{i}(\omega) \tau_{1}(\omega) P_{1}(\omega)\left(1+r_{1-2}(\omega) r_{1-a i r}(\omega) P_{1}^{2}(\omega)\right)
$$

donde $E_{i}(\omega)=\left|E_{i}(\omega)\right| e^{j \theta_{i}(\omega)}$, es la componente del campo eléctrico dentro del primer material, $\tau_{1}(\omega)=\frac{2 n_{1}(\omega)}{n_{1}+n_{2}}, r_{1-2}=\frac{n_{1}-n_{2}}{n_{1}+n_{2}}, r_{1-a i r}=\frac{n_{1}-1}{n_{1}+1}$ y $P_{1}=e^{-j \frac{n_{1} \omega d_{1}}{c}}$ son los coeficientes de trasmisión, reflexión y propagación respectivamente, siendo $n_{1}$ y $d_{1}$ el índice de refracción y espesor del primer material.

El campo eléctrico luego del segundo material, se puede escribir como: 


$$
\begin{aligned}
E_{t 2}(\omega)= & E_{t i}(\omega) \tau_{2}(\omega) P_{2}(\omega)\left(1+r_{2-1}(\omega) r_{2-a i r}(\omega) P_{2}^{2}(\omega)\right) \\
& =E_{i}(\omega) \tau_{1}(\omega) P_{1}(\omega) \tau_{2}(\omega) P_{2}(\omega) \\
& +E_{i}(\omega) \tau_{1}(\omega) r_{1-2}(\omega) r_{1-a i r}(\omega) P_{1}^{3}(\omega) \tau_{2}(\omega) P_{2}(\omega) \\
& +E_{i}(\omega) \tau_{1}(\omega) P_{1}(\omega) \tau_{2}(\omega) r_{2-1}(\omega) r_{2-a i r}(\omega) P_{2}^{3}(\omega) \\
& +E_{i}(\omega) \tau_{1}(\omega) r_{1-2}(\omega) r_{1-a i r}(\omega) P_{1}^{3}(\omega) \tau_{2}(\omega) r_{2-1}(\omega) r_{2-a i r}(\omega) P_{2}^{3}(\omega)
\end{aligned}
$$

con $\tau_{2}(\omega), \tau_{2-1}(\omega), \tau_{2-a i r}(\omega)$ y $P_{2}(\omega)$ los coeficientes de transmisión, reflexión y propagación del segundo material, siendo $n_{2}$ y $d_{2}$ el índice y espesor correspondiente. El último término de la ecuación (5.14) se desprecia ya que tiene baja amplitud frente a los otros términos. Esto ocurre porque pertenece al eco generado en el segundo material, por el eco que se generó en el primero.

Realizando el módulo al cuadrado de la ecuación (5.14), asumiendo los índices y el campo eléctrico constantes en frecuencia, se obtiene:

$$
\begin{aligned}
\left|E_{t 2}(\omega)\right|^{2}= & H_{1}\left(n_{1}, n_{2}\right)+H_{2}\left(n_{1}, n_{2}\right) \cos \left(\frac{2 n_{1} \omega d_{1}}{c}\right)+H_{3}\left(n_{1}, n_{2}\right) \cos \left(\frac{2 n_{2} \omega d_{2}}{c}\right) \\
& +H_{4}\left(n_{1}, n_{2}\right) \cos \left(\frac{2 \omega}{c}\left(n_{1} d_{1}-n_{2} d_{2}\right)\right) .
\end{aligned}
$$

Definiendo $C_{1}=\tau_{1} \tau_{2} r_{1-2} r_{1-a i r}, C_{2}=\tau_{1} \tau_{2}$, y $C_{3}=\tau_{1} \tau_{2} r_{2-1} r_{2-a i r}$ los coeficientes $H_{i}\left(n_{1}, n_{2}\right)$ pueden escribirse como: $H_{1}=C_{1}^{2}+C_{2}^{2}+C_{3}^{2}, H_{2}=2 C_{1} C_{2}$, $H_{3}=2 C_{2} C_{3}$ y $H_{4}=2 C_{1} C_{3}$.

Aplicando la transformada de Fourier a la ecuación (5.15), se obtiene:

$$
\begin{aligned}
I(t)= & \mathcal{F}\left(\left|E_{t 2}(\omega)\right|^{2}\right)=H_{1}\left(n_{1}, n_{2}\right) \delta(t)+\frac{H_{2}\left(n_{1}, n_{2}\right)}{2} \delta\left(t-\frac{2 n_{1} d_{1}}{c}\right) \\
& +\frac{H_{3}\left(n_{1}, n_{2}\right)}{2} \delta\left(t-\frac{2 n_{2} d_{2}}{c}\right)+\frac{H_{4}\left(n_{1}, n_{2}\right)}{2} \delta\left(\frac{2 \omega}{c}\left|n_{1} d_{1}-n_{2} d_{2}\right|\right) .
\end{aligned}
$$

Los últimos tres términos de la ecuación (5.16) muestran que la posición de las deltas de Dirac dan una relación entre los índices de refracción, y los espesores. Aunque sólo dos de los tres, ofrecen ecuaciones independientes ya que el desplazamiento del cuarto término puede escribirse como una combinación lineal de los otros dos. Entonces, tomando el cociente de las amplitudes del 


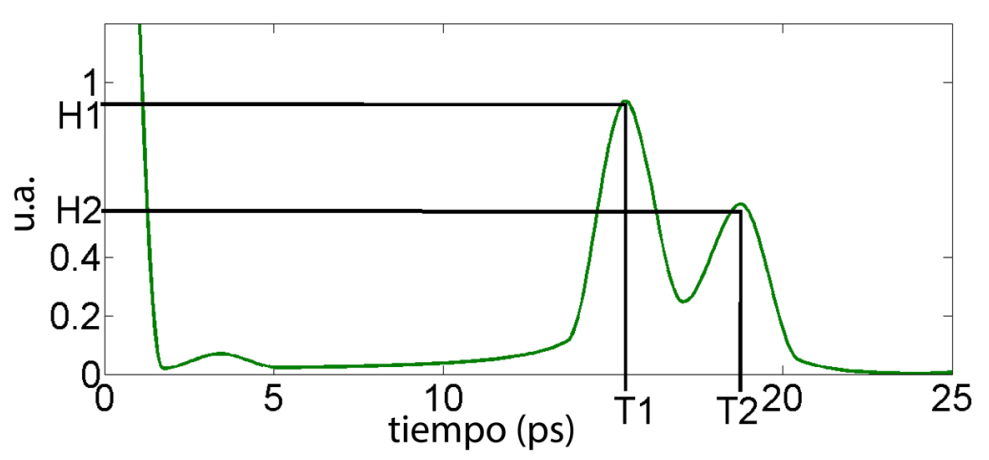

Figura 5.10: Simulación numérica de la transformada discreta de Fourier aplicada a la ecuación (5.15).

segundo y tercer término, se obtiene una tercera ecuación independiente que relaciona dichas variables. El sistema formado por las ecuaciones (5.17)- (5.20) es el utilizado para obtener las variables $n_{1}, n_{2}, d_{1}, d_{2}$, donde $d$ en la ecuación (5.20) es la medida del espesor de toda la estructura.

$$
\begin{gathered}
T_{1}=\frac{2 n_{1} d_{1}}{c} \\
T_{2}=\frac{2 n_{2} d_{2}}{c} \\
\frac{H_{2}\left(n_{1}, n_{2}\right)}{H_{3}\left(n_{1}, n_{2}\right)}=\frac{r_{1-a i r}(\omega)}{r_{2-a i r}(\omega)}=\frac{\left(n_{1}-1\right)\left(n_{2}+1\right)}{\left(n_{1}+1\right)\left(n_{2}-1\right)} \\
d=d_{1}+d_{2} .
\end{gathered}
$$

Como el experimento tiene trazas temporales finitas, se obtienen pulsos de cierto ancho en vez de deltas. El ensanche de los pulsos limita la resolución del enfoque propuesto.

La Figura 5.10 muestra una simulación numérica con $n_{1}=3,5, n_{2}=1,82$, $d_{1}=670 \mu \mathrm{m}, d_{2}=1570 \mu \mathrm{m}$, asumiendo un ancho de banda de $1 \mathrm{THz}$.

En dicha figura se observan los 4 pulsos de la ecuación (5.16), donde los más alejados del origen facilitan $T_{1}, T_{2}, H_{2}$, y $H_{1}$, los cuales son utilizados para resolver el sistema de ecuaciones. Los errores en la extracción de parámetros esta dada por el error cometido en la medición del espesor de la estructura, por el ancho de banda del espectrómetro, y por el ruido que afecta las amplitudes de los picos. En el último caso, se realizaron simulaciones considerando un sistema con una relación señal a ruido de $30 \mathrm{~dB}$, que es un valor que esta por debajo de los valores típicos de los espectrómetros de Terahertz [59]. Como resultado se 

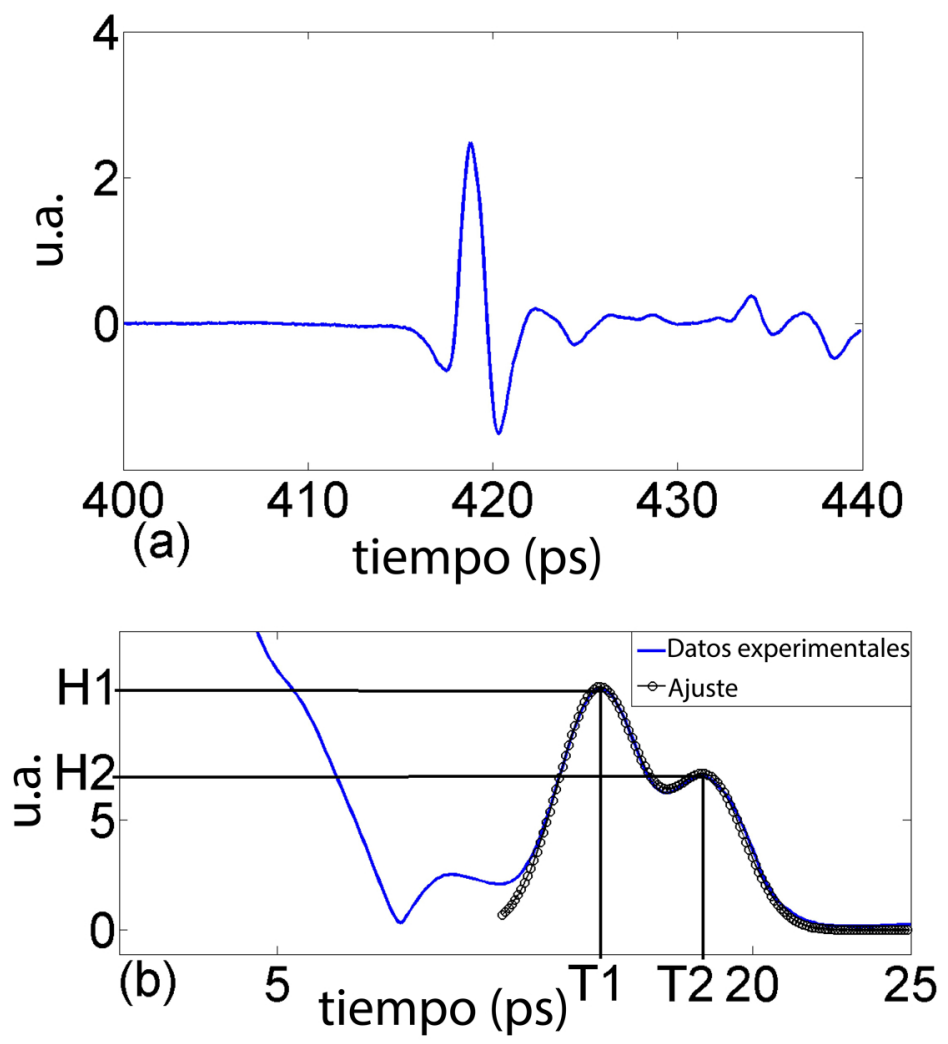

Figura 5.11: (a) Traza temporal medida del espectómetro de THz. (b) Resultado de aplicar el procesamiento propuesto a la traza temporal mostrada en (a).

obtuvieron variaciones menores al 0,2\%, siendo despreciada su influencia frente a los otros errores.

\subsubsection{Experimento y resultados}

El experimento fue realizado mediante el uso del espectrómetro de $\mathrm{THz}$ del Centro de Tecnología Nanofótonica de la Universidad Politécnica de Valencia descripto en la sección 5.2.4. Las medidas se realizaron con un barrido temporal de 40 ps, con un paso de 100 fs. La estructura bicapa consistía en un polímero (polybutylene terephthalate (PBT)) con una oblea de silicio. La Figura 5.11(a) muestra la traza temporal medida del espectrómetro. En la misma se observa el pulso principal, y dos picos mostrando reflexiones alrededor de 434 ps y 437 ps.

La Figura 5.11(b) muestra el resultado de aplicar el procesamiento descripto en la teoría a la traza temporal mostrada en la Fig. (5.11)(a). En la misma, se 
observan dos picos aparte del pulso observado en el origen en vez de los tres representados en la ecuación (5.16). Esto se debe a que el pico correspondiente al último término en (5.16) es el resultado de la diferencia entre los retardos temporales introducidos por cada material. En el caso en que $T_{1}$ y $T_{2}$ son similares, $T_{2}-T_{1}$ es cercano a cero, no pudiéndolo distinguir en la figura. Esto se ha generado a causa de que el ancho de banda experimental no fue suficiente para distinguir el último término con el primero de la ecuación (5.16).

También se observa en la Fig. 5.11(b) que las amplitudes de los picos $\left(H_{1}\right.$ y $\mathrm{H}_{2}$ ) utilizadas para obtener los parámetros desconocidos, son afectados por las colas de dichos pulsos debido a la falta de resolución espectral. Para mejorar los resultados se realizó un ajuste de la señal por una función compuesta de la suma de dos funciones Gaussianas (ver Fig. 5.11(b)), de las cuales se extrajo la amplitud de la señal. Finalmente, reemplazando los valores obtenidos en la Fig. 5.11(b) ( $T_{1}$ y $T_{2}$ ), los valores de los picos de las Gaussianas del ajuste, y la medida del ancho total de la estructura multicapa en el sistema de ecuaciones, se obtuvo:

$$
T_{1}=15,4 \mathrm{ps}, T_{2}=18,9 \mathrm{ps}, \frac{H_{1}}{H_{2}}=1,9, d=2220 \mu \mathrm{m} .
$$

Resolviendo numéricamente el sistema de ecuaciones son obtenidos dos resultados. Solo uno es válido, ya que el otro da como resultado índices menores a uno. Los resultados son:

$$
n_{1}=3,48, n_{2}=1,82, d_{1}=664 \mu \mathrm{m}, d_{2}=1556 \mu \mathrm{m} .
$$

El error experimental puede calcularse numéricamente adicionando la dispersión provocada por la resolución temporal, y por el error cometido al medir el ancho total de la muestra. Por lo tanto, reemplazando en el sistema de ecuaciones $T_{1}$ por $T_{1} \pm 50 \mathrm{fs}, T_{2}$ por $T_{2} \pm 50 \mathrm{fs}$ y $d$ por $d \pm 10 \mu \mathrm{m}$, el error en los resultados fueron de:

$$
\triangle n_{1}= \pm 0,11, \triangle n_{2}= \pm 0,06, \triangle d_{1}= \pm 11 \mu \mathrm{m}, \mathrm{y} \triangle d_{2}= \pm 0,16 \mu \mathrm{m}
$$

Los resultados obtenidos fueron verificados midiendo y procesando los datos de cada muestra por separado de manera convencional, obteniendo como índice promedio para el silicio $n_{1}=3,5$, y $n_{2}=1,8$ para la muestra PBT. Los espesores medidos de cada capa con el uso de un calibre fueron $640 \mu \mathrm{m}$ para la oblea de silicio y $1570 \mu \mathrm{m}$ para el PBT. Con dichos resultados se puede evidenciar la correspondencia de los valores verificando la validez del método propuesto. 


\subsubsection{Conclusión}

Mediante el presente trabajo se propuso y se demostró el buen funcionamiento de un método simple para determinar los parámetros ópticos, y espesores de una estructura bicapa mediante la realización de una sola medida.

El método utiliza el resultado de la medida espectral de la muestra, y la medida del espesor total de la estructura para obtener los índices de la misma. En el caso en que el índice no sea constante en el ancho de banda del espectrómetro, los resultados pueden ser utilizados como valores iniciales para el uso de otros algoritmos. Por último, cabe mencionar que la técnica propuesta es útil para el estudio no invasivo de semiconductores, materiales compuestos, etc. 


\section{Capítulo 6}

\section{Conclusiones y posibilidades futuras}

\subsection{Conclusiones}

El objetivo principal de la tesis fue el desarrollo de un espectrómetro de THz. Éste fue realizado mediante el uso de un láser de pulsos ultracortos de luz, y antenas fotoconductoras. Dicho trabajo, nos llevó a ser pioneros en el país en generar y detectar señales con tales características.

Para lograr el objetivo se hicieron implementaciones en software, hardware, e incluso en diseño de piezas mecánicas.

Durante el proceso se logró innovar en un método de medición para caracterizar el ancho temporal de los pulsos láser de femtosegundos necesarios para la generación de $\mathrm{THz}[10]$.

Una vez finalizada la etapa de desarrollo del espectrómetro, se obtuvieron satisfactoriamente espectros del vapor de agua y de materiales mediante el uso de algoritmos de procesamiento convencionales.

En una segunda parte del proyecto, se buscó innovar en técnicas de procesamiento de datos aplicados a la obtención de los parámetros ópticos de materiales. Tal propósito, generó tres publicaciones en revistas internacionales.

La primera posibilita el análisis de materiales monocapa [11] mediante el uso de una sola medida del espectrómetro, otorgando como principal característica mayor inmunidad al ruido que los métodos de obtención de parámetros por observación temporal directa.

La segunda permite trabajar con materiales birrefringentes [12], logrando con el uso de una sola medida obtener ambos índices de refracción, y con otra medida más la posición del eje óptico. Esto es muy innovador porque los métodos comúnmente utilizados necesitan para lograr obtener dichos parámetros al 
menos cuatro mediciones.

La última publicación se basa en la obtención de los parámetros ópticos de una estructura bicapa [13], con la cual se obtiene los dos índices de refracción y espesores de la estructura, solamente con el uso de los datos obtenidos de una medición del espectrómetro y con el conocimiento del espesor total de la estructura. El trabajo ha sido innovador porque no hay antecedentes de que con una sola medida se pueda obtener dichos paramétros de una estructura bicapa.

Cabe mencionar que durante el transcurso de la tesis, se logró establecer cooperación con el grupo de THz del Centro de Tecnología Nanofotónica de la Universidad Politécnica de Valencia, posibilitando trabajos conjuntos.

El resultado final y principal de la tesis fue la obtención de un conocimiento de la temática, logrando la capacidad de desarrollar proyectos experimentales.

\subsection{Posibilidades futuras}

Las posibilidades futuras que evidenciamos no están dadas mayormente por un cambio en la implementación experimental, sino en la mejora de las señales mediante la innovación en procesamiento de datos, ya que poco se ha trabajado en dichos aspectos. Se podrían realizar propuestas para disminuir el ruido en la señal con filtros digitales, también se podría mejorar la resolución espectral mediante el uso de enfoques diferentes a la trasformada de Fourier o Wavelets que son los utilizados actualmente. Asimismo, se podría realizar un análisis de los errores experimentales intentando cuantificarlos mediante diferentes algoritmos, como puede ser la cota de Cramér-Rao. 


\section{Apéndice A}

\section{Anexo I: Programa de adquisición de datos}

$\mathrm{s}=\operatorname{serial}($ 'COM1'); set(s,'BaudRate', 9600,'Parity','none','StopBits',1,

'FlowControl','none','DataBits',8,'Terminator','CR');

get(s)

fopen $(\mathrm{s})$;

RRU =fopen('pruebaX.txt', 'wt');

$\mathrm{RRa}=$ fopen( 'pruebaY.txt', 'wt');

fprintf(s,'OUTX 0');

fprintf(s,'FMOD 1;FREQ 30000;RSLP 1');

fprintf(s,'ISRC 2;IGND 1;ICPL 1;ILIN 3');

fprintf(s,'SENS 15;RMOD 1;OFLT 7;OFSL 0;SYNC 0');

fprintf(s,'DDEF 1,0,0;DDEF 2,0,0;FPOP 1,1;FPOP 2,1;OEXP 1,0,0');

fprintf(s,'SRAT 9;SEND 0');

fprintf(s,'REST');

pause on

$\mathrm{M}=16300$;

$\mathrm{i}=1$;

$\mathrm{I}(\mathrm{M})=5 \mathrm{E}-9$;

$\mathrm{U}(\mathrm{M})=0$;

for $(\mathrm{i}=1: 18: \mathrm{M})$,

if $(\mathrm{i}==1)$,

fprintf(s,'STRT');

pause(3);

end;

fprintf(s,'SPTS?');

out1 $=$ fscanf $(\mathrm{s})$;

$\mathrm{L}=$ str2num(out1); 


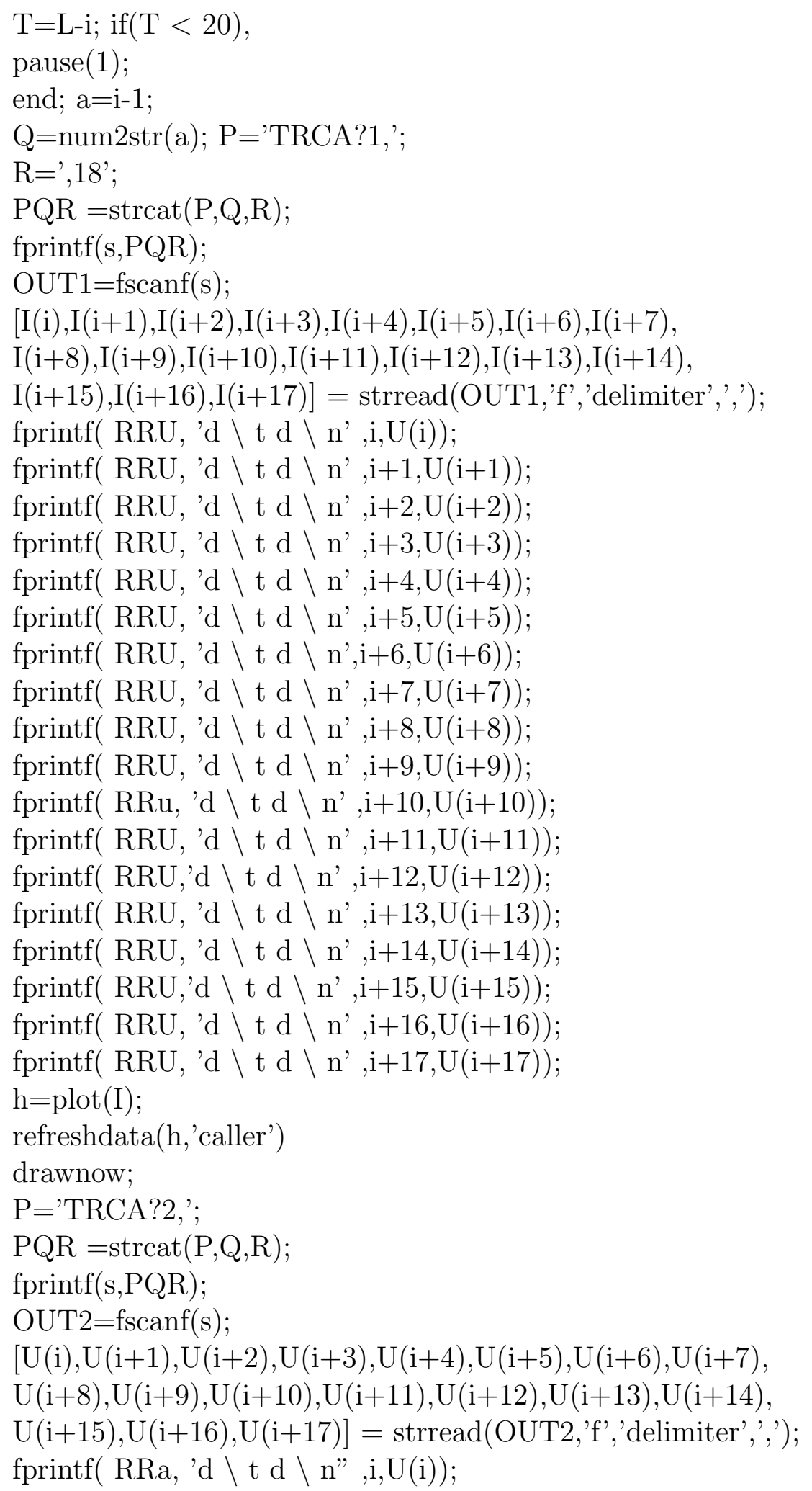




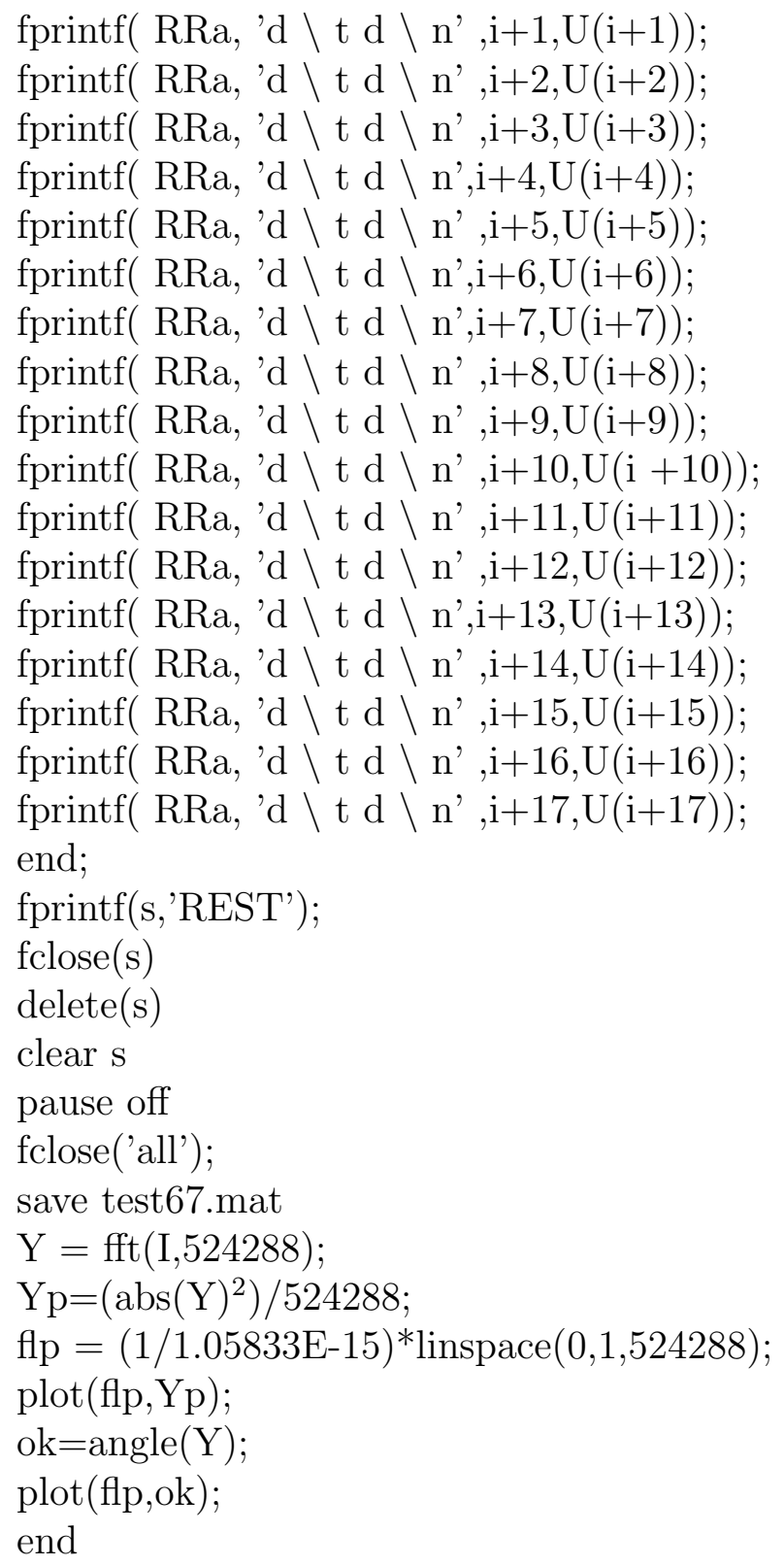


90 APÉNDICE A. ANEXO I: PROGRAMA DE ADQUISICIÓN DE DATOS 


\section{Bibliografía}

[1] Yun Shik Lee. Principles of terahertz science. Springer, 2009.

[2] Susan L. Dexheimer. Terahertz spectroscopy. CRC, 2008.

[3] S. Withington. Terahertz astronomical telescopes and instrumentation. Philosophical Transactions of the Royal Society of London. Series A: Mathematical, Physical and Engineering Sciences, 362:395,402, 2004.

[4] I. Kallfass, J. Antes, T. Schneider, F. Kurz, D. Lopez Diaz, S. Diebold, H. Massler, A. Leuther, and A. Tessmann. All active mmic-based wireless communication at 220 ghz. IEEE Transactions on Terahertz Science and Technology, 1:477,487, 2011.

[5] D. H. Auston, A. M. Johnson, P. R. Smith, and J. C. Bean. Picosecond optoelectronic detection, sampling and correlation measurements in amorphous semiconductors. Applied Physics Letter, 37:371,373, 1980.

[6] Xi Cheng Zhang and Jingzhou Xu. Introduction to THz Wave Photonics. Springer, 2010.

[7] C. Jördens and M. Koch. Detection of foreign bodies in chocolate with pulsed terahertz spectroscopy. Optical Engineering, 47, 2007.

[8] D. M. Mittleman, M. Gupta, R. Neelamani, R. G. Baraniuk, J. V. Rudd, and M. Koch. Recent advances in terahertz imaging. Applied Physics B, 68:1085,1094, 1999.

[9] M. C. Kemp. Explosives detection by terahertz spectroscopy - a bridge too far? IEEE Transactions on Terahertz Science and Technology, 1:282,292, 2011.

[10] F. Sanjuan, J. Tocho, and B. Vidal. Spatial measurement of ultrashort light pulses using two photon absorption in a colliding geometry. Trabajo enviado a OPTIK Journal, 2014. 
[11] F. Sanjuan and B. Vidal. Refractive index calculation from echo interference in pulsed terahertz spectroscopy. Electronics Letters, 50:308,309, 2014 .

[12] F. Sanjuan, A. Bockelt, and B. Vidal. Birefringence measurement in the terahertz range based on double fourier analysis. Optics Letters, 39:809,812, 2014.

[13] F. Sanjuan, A. Bockelt, and B. Vidal. Determination of refractive index and thickness of a multilayer structure with a single thz time domain spectroscopy measurement. Applied Optics, 53:4910,4913, 2014.

[14] Peter H. Siegel. Thz instruments for space. IEEE Transactions on Antennas and Propagation, 55:2957,2965, 2007.

[15] Herman A. Haus. Mode-locking of lasers. IEEE Journal on Selected Topics in Quantum Electronics, 6:1173,1185, 2000.

[16] M. Ghanashyam Krishna, Sachin D. Kshirsagar, and Surya P. Tewari. Terahertz Emitters, Detectors and Sensors: Current Status and Future Prospects, Photodetectors. InTEch, 2012.

[17] T. W. Crowe, W. L. Bishop, D. W. Porterfield, J. L. Hesler, and R. M. Weikle. Opening the terahertz window with integrated diode circuits. IEEE Journal of Solid-State Circuits, 40:2104,2110, 2005.

[18] Y. Shen, T. Watanabe, D. A. Arena, C. Kao, J. B. Murphy, T. Y. Tsang, X. J. Wang, and G. L. Carr. Nonlinear cross-phase modulation with intense single-cycle terahertz pulses. Physical Review Letters, 99:1,4, 2007.

[19] Shengqi Xu, Yizhu Zhang, Yinbo Zheng, and Weiwei Liu. Study of physical mechanism of two-color laser field pumped thz wave in air plasma. Terahertz Science and Technology, 3:130,142, 2010.

[20] K. Y. Kim, J. H. Glownia, A. J. Taylor, and G. Rodriguez. Terahertz emission from ultrafast ionizing air in symmetry-broken laser fields. Optics Express, 15:4577,4584, 2007.

[21] Maxim A. Khazan. Time domain Terahertz spectrocopy and its application to the study of high-Tc superconductor thin films. PhD thesis, Universidad de Hamburgo, 2002.

[22] Justin T. Darrow, Xi-Cheng Zhang, David H. Auston, and Jeffrey D. Morse. Saturation properties of large-aperture photoconducting antennas. IEEE Journal of Quantum Electronics, 28:1607,1616, 1992. 
[23] S. D. Ganichev and W. Prettl. Intense Terahertz Excitation of Semiconductors. Oxford Univ., 2006.

[24] Masahiko Tani, Shuji Matsuura, Kiyomi Sakai, and Shin ichi Nakashima. Emission characteristics of photoconductive antennas based on low-temperature-grown gaas and semi-insulating gaas. Applied Optics, $36: 7853,7859,1997$.

[25] H. Tanoto, J. H. Teng, Q.Y.Wu, M.Sun, Z. N. Chen, S. A. Maier, B. Wang, C. C. Chum, G.Y.Si, A. J. Danner, and S. J. Chua. Nano-antenna in a photoconductive photomixer for highly efficient continuous wave terahertz emission. Scientific Reports, 2013.

[26] S. Verghese, K. A. McIntosh, S. Calawa, W. F. Dinatale, E. K. Duerr, and K. A. Molvar. Generation and detection of coherent terahertz waves using two photomixers. Applied Physics Letter, 73:3824,3826, 98.

[27] A. Rogalski and F. Sizov. Terahertz detectors and focal plane arrays. Opto Electronics Review, 2011:346,404, 1998.

[28] J. Zmuidzinas, A. Karpov, D. Miller, F. Rice, Henry Leduc, J. Pearson, and J. A. Stern. Coherent detection and sis mixers. Far IR, Sub-mm and MM Detector Technology Workshop, pages 1,6, 2002.

[29] X. Xie, J. Dai, and X. Zhang. Coherent control of thz wave generation in ambient air. Physical Review Letters, 96:1,4, 2006.

[30] P. Jepsen, D. Cooke, and M. Koch. Terahertz spectroscopy and imaging-modern techniques and applications. Laser Photonics Review, $5: 124,166,2011$.

[31] G. Chattopadhyay. Technology, capabilities, and performance of low power terahertz sources. IEEE Transactions on Terahertz Science and Technology, 1:33,53, 2011.

[32] C. M. Armstrong. The truth about terahertz. IEEE Spectrum, 49:36,41, 2012.

[33] B. B. Hu and M. C. Nuss. Imaging with terahertz waves. Optics Letters, 20:1716,1718, 1995.

[34] S. Wietzke, C. Jördens, N. Krumbholz, B. Baudrit, M. Bastian, and M. Koch. Terahertz imaging: a new non-destructive technique for the quality control of plastic weld joints. Journal of the European Optical Society - Rapid publications, 2, 2007. 
[35] J. Jackson, M. Mourou, J. Labaune, J. Whitaker, I. Duling, S. Williamson, C. Lavier, M. Menu, and G. Mourou. Terahertz pulse imaging for tree-ring analysis: a preliminary study for dendrochronology applications. Measurement Science and Technology, 20, 2009.

[36] L. Titova, A. Ayesheshim, A. Golubov, D. Fogen, and O. Kovalchuk R. Rodriguez-Juarez, F. Hegmann. Intense thz pulses cause h2ax phosphorylation and activate dna damage response in human skin tissue. Biomedical Optics Express, 4:559,568, 2013.

[37] K. Kawase, Y. Ogawa, Y. Watanabe, and H. Inoue. Non-destructive terahertz imaging of illicit drugs using spectral fingerprints. Optics Express, $11: 2549,2554,2003$.

[38] M. Scheller, C. Jansen, and Martin Koch. Recent Optical and Photonic Technologies. InTEch, 2010.

[39] Z. A. Yasa and N. M. Amer. A rapid scanning autocorrelation scheme for continuous monitoring of picosecond laser pulses. Optic Communication, 36:406,408, 1981.

[40] J. H. Chung and A. M. Weiner. Ambiguity of ultrashort pulse shapes retrieved from the intensity autocorrelation and the power spectrum. Quantum Electronics, 7:656,666, 2001.

[41] J. Etchepare, G. Grillon, and A. Orszag. Third order electronic susceptibilities of liquids measured by femtosecond kinetics of optical kerr effect. Quantum Electronics, 19:775,778, 1983.

[42] R. Trebino. Frequency Resolved Optical Gatin: The Measurements of Ultrashort laser Pulses. Kluwer Academic Publishers, 2000.

[43] R. Salem. Characterization of two-photon absorption detectors for applications in high-speed optical systems. Master's thesis, Universidad de Maryland, 2003.

[44] M. Raghuramaiah, A. K. Sharma, P.A. Naik, P. D. Gupta, and R. A. Ganeev. A second-order autocorrelator for single-shot measurement of femtosecond laser pulse durations. Sadhana, pages 1603,1611, 2001.

[45] P. A. Franken, A. E. Hill, C. W. Peters, and G. Weinreich. Generation of optical harmonics. Physics Review Letters, 7:118,119, 1961. 
[46] D. T. Reid, W. Sibbett, J. M. Dudley, L. P. Barry, B. Thomsen, and J. D. Harvey. Commercial semiconductor devices for two photon absorption autocorrelation of ultrashort light pulses. Applied Optics, 37:8142,8144, 1998.

[47] M. Rasmusson, A. N. Tarnovsky, E. Akesson, and V. Sundstrom. On the use of two-photon absorption for determination of femtosecond pump probe cross correlation functions. Chemical Physics Letters, 335:201,208, 2001.

[48] L.P. Barry, K. Bondarczuk, K. J. Dexter, P.J. Maguire, D.A. Reid, J. O Dowd, W.H. Guo, M. Lynch, A.L. Bradley, and J.F. Donegan. All-optical pulse processing for advanced photonic communication system. Proceedings of the 10th Anniversary International Conference on: Transparent Optical Networks (ICTON), 40:92,95, 2008.

[49] D. J. Bradley and H. C. Geoffrey. Ultrashort pulse measurements. Proceedings of the IEEE (SPIE), pages 313,345, 1974.

[50] O. Ollikainen, A. Rebane, D. Erni, and U. P. Wild. Femtosecond timeresolved pulse measurement and image detection in broad spectral range by two-photon-excited fluorescence. Lasers and Electro Optics, 335:227,228, 1996.

[51] A. Penzkofer O. Lammel. Femtosecond pulse duration measurement by two photon fuorescence detection. Optical and Quantum Electronics, $32: 1147,1160,2000$.

[52] P. Sperber and A. Penzkofer. Pulse-shape determination of intracavity compressed picosecond pulses by two-photon fluorescence analysis. Optical and Quantum Electronics, 18:145,154, 1986.

[53] D. Narayana Rao N. K. M Naga Srinivas, S. Venugopal Rao. Saturable and reverse saturable absorption of rhodamine $\mathrm{b}$ in methanol and water. JOSA Society of America B, 20:2470,2479, 2003.

[54] N. Llombart and A. Neto. Thz time-domain sensing: The antenna dispersion problem and a possible solution. IEEE Transactions on Terahertz Science and Technology, 2:416,423, 2012.

[55] N. Katzenellenbogen and D. Grischkowsky. Efficient generation of 380 fs pulses of thz radiation by ultrafast laser pulse excitation of a biased metalsemiconductor interface. Applied Physics Letters, 58:222,224, 1991.

[56] M.V. Exter, Ch. Fattinger, and D. Grischkowsky. Terahertz time domain spectroscopy of water vapor. Optics letters, 14:1128,1130, 1989. 
[57] X. Xin, H. Altan, A. Saint, D. Matten, and R. R. Alfano. Terahertz absorption spectrum of para and ortho water vapors at different humidities at room temperature. Applied Physics, 100, 2006.

[58] F. Sanjuan and J. Tocho. Generation and measurement of terahertz radiation using ultrashort light pulses. Anales AFA, 23:65,70, 2011.

[59] L. Duvillaret, F. Garet, and J. L. Coutaz. A reliable method for extraction of material parameters in terahertz time-domain spectroscopy. IEEE Journal of Selected Topics in Quantum Electronics, 2:739,746, 1996.

[60] W. Withayachumnankul, B. Ferguson, T. Rainsford, S. P. Mickan, and D. Abbott. Material parameter extraction for terahertz time-domain spectroscopy using fixed-point iteration. Proc. of SPIE, pages 221,231, 2005.

[61] L. Duvillaret, F. Garet, and J. L. Coutaz. Influence of noise on the characterization of materials by terahertz time-domain spectroscopy. JOSA Society of America B, 17:452,461, 2000.

[62] L. Duvillaret, F. Garet, and J. L. Coutaz. Highly precise determination of optical constants and sample thickness in terahertz time-domain spectroscopy. Applied Optics, 38:409,415, 1999.

[63] S.K. Grischkowsky, M. Van Exter, and C. Fattinger. Far infrared time domain spectroscopy with terahertz beams of dielectrics and semiconductor. JOSA Society of America B, 7:2006,2015, 1990.

[64] F. Sanjuan and J. Tocho. Optical properties of silicon, sapphire, silica and glass in the terahertz range. Latin America Optics and Photonics Conference (LAOP), (OSA), 2012.

[65] M.V. Exter and D. Grischkowsky. Characterization of an optoelectronic terahertz beam system. IEEE Transactions on Microwave Theory and Techniques, 38:1684,1691, 1990.

[66] N. Wang and M. Jarrahi. Noise analysis of photoconductive terahertz detectors. Journal of Infrared, Millimeter, and Terahertz Waves, 34:519,528, 2013.

[67] L. Hou, W. Shi, and S. Chen. Noise analysis and optimization of terahertz photoconductive emitters. IEEE Journal of Selected Topics in Quantum Electronics, 19, 2013. 
[68] K. Wiesauerand and C. Jordens. Recent advances in birefringence studies at thz frequencies. Journal of Infrared, Millimeter, and Terahertz Waves, 34:663,681, 2013.

[69] S. Ebara, Y. Hirota, M. Tani, and M. Hangyo. Highly sensitive birefringence measurement in thz frequency region and its application to stress measurement. IRMMW-THz. Joint 32nd International Conference (Springer), 2007.

[70] C. Jördens, M. Scheller, S. Wietzke, D. Romeike, C. Jansen, T. Zentgraf, K. Wiesauer, V. Reisecker, and M. Koch. Terahertz spectroscopy to study the orientation of glass fibres in reinforced plastics. Composites Science and Technology, 70:472,477, 2010.

[71] C. Jördens, M. Scheller, M. Wichmann, M. Mikulics, K. Wiesauer, and M. Koch. Terahertz birefringence for orientation analysis. Applied Optics, 48:2037,2044, 2009.

[72] 5. Y. Kim, M. Yi, B. Kim, and J. Ahn. Investigation of thz birefringence measurement and calculation in al2o3 and linbo3. Applied Optics, 50:2906,2910, 2011.

[73] M. Reid and R. Fedosejevs. Terahertz birefringence and attenuation properties of wood and paper. Applied Optics, 45:2766,2772, 2006.

[74] S. Katletz, M. Pfleger, H. Uhringer, M. Mikulics, N. Vieweg, O. Peters, B. Scherger, M. Scheller, M. Koch, and K. Wiesauer. Polarization sensitive terahertz imaging: detection of birefringence and optical axis. Optics Express, 20:23025,23035, 2012.

[75] H. G. Tompkins and E. A. Irene. Handbook of Ellipsometry. Springer, 2005.

[76] A. Yu. Nikulin and P. V. Petrashen. Practical applications of optical inverse problem technique to characterization of multilayers. Denver X-ray Conference (AXA), 1997.

[77] S. E. Ralph, S. Perkowitz, N. Katzenellenbogen, and D. Grischkowsky. Terahertz spectroscopy of optically thick multilayered semiconductor structures. Journal of the Optical Society of America B, 11:2528,2532, 1994.

[78] R. Wilk, I. Pupeza, R. Cernat, and M. Koch. Highly accurate thz timedomain spectroscopy of multilayer structures. IEEE Journal of Selected Topics in Quantum Electronics, 14:392,398, 2008. 
[79] J. A. Hejase, E. J. Rothwell, and P. Chahal. A multiple angle method for thz time-domain material characterization. IEEE Transactions on Terahertz Science and Technology, 3:656,665, 2013.

[80] A. Brahm, A. Weigel, S. Riehemann, G. Notni, and A. Tunnermann. Highly precise parameter extraction of thin multilayers in thz transmission and reflection geometry. IEEE Conference on Infrared, Millimeter and Terahertz Waves, 2011. 